TAILORING PULSES FOR COHERENT RAMAN MICROSCOPY

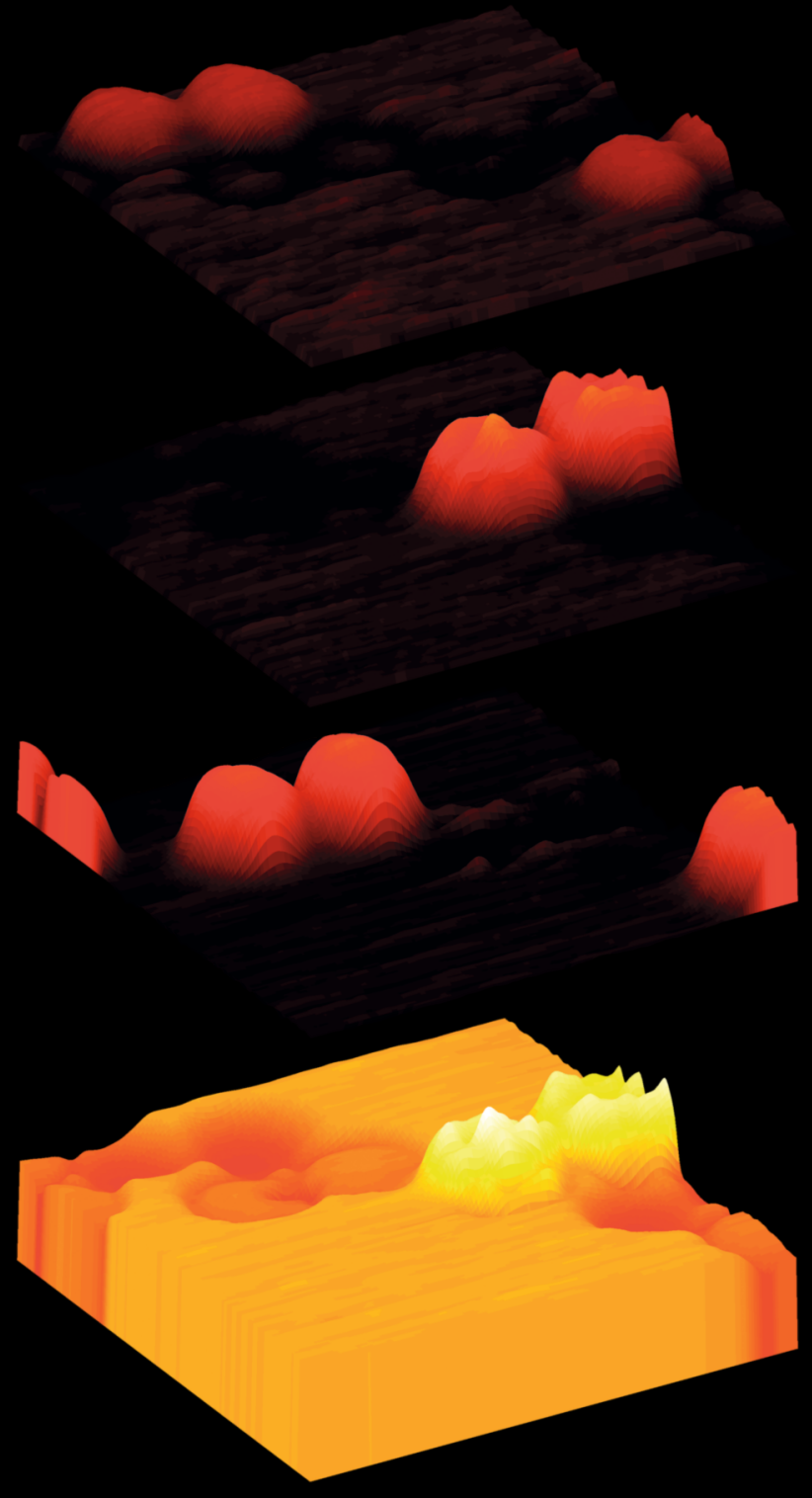

ALEXANDER VAN RHIJN 



\section{Tailoring Pulses for Coherent RAMAN Microscopy}


Samenstelling van de promotiecommissie:

Prof. dr. G. van der Steenhoven Universiteit Twente, Enschede, Nederland Prof. dr. J.L. Herek

Universiteit Twente, Enschede, Nederland

Dr. ir. H.L. Offerhaus

Universiteit Twente, Enschede, Nederland

Prof. dr. K.J. Boller

Prof. dr. A.P. Mosk

Universiteit Twente, Enschede, Nederland

Prof. dr. Y. Silberberg Universiteit Twente, Enschede, Nederland

Prof. dr. M.A.G.J. Orrit

Weizmann Institute of Science, Rehovot, Israël Universiteit Leiden, Leiden, Nederland

This research was supported by the IOP (Innovatiegerichte

Onderzoeksprogramma's) Photonic Devices program managed by the Technology Foundation STW (Stichting Technische Wetenschappen) and Agentschap NL.

This work was carried out at:

Optical Sciences group, MESA ${ }^{+}$Institute for Nanotechnology, Faculty of Science and Technology (TNW), University of Twente, The

Netherlands

Cover design: Alexander van Rhijn

Photo by: Martin Jurna

ISBN: 978-90-365-3390-4

Printed by: Gildeprint Drukkerijen - The Netherlands

Author email: alexandervanrhijn@gmail.com

Copyright (C) 2012 by Alexander van Rhijn

All rights reserved. No part of the material protected by this copyright notice may be reproduced or utilized in any form or by any means, electronic or mechanical, including photocopying, recording or by any information storage and retrieval system, without the prior permission of the author. 


\section{Tailoring Pulses for Coherent RAMAN MicRoscopy}

\section{PROEFSCHRIFT}

ter verkrijging van

de graad van doctor aan de Universiteit Twente, op gezag van de rector magnificus,

prof. dr. H. Brinksma,

volgens besluit van het College voor Promoties

in het openbaar te verdedigen

op vrijdag 20 juli 2012 om 12.45 uur

door

Alexander Cornelis Willem van Rhijn

geboren op 9 juli 1985

te 's-Gravendeel, Nederland 
Dit proefschrift is goedgekeurd door:

Prof. dr. J.L. Herek (Promotor)

Dr. ir. H.L. Offerhaus (Assistent promotor) 


\section{Contents}

1 Introduction 1

1.1 Optical microscopy . . . . . . . . . . . . . . . . . . 1

1.2 Phase and interference . . . . . . . . . . . . . 3

1.3 Nonlinear microscopy _. . . . . . . . . . . . . . . 4

1.4 Thesis overview . . . . . . . . . . . . . 6

2 Broadband coherent Raman scattering 9

2.1 Interaction of light and matter . . . . . . . . . . 10

2.1 .1 Introduction . . . . . . . . . . . . . 10

2.1.2 Spontaneous Raman scattering . . . . . . . . . . 11

2.2 Coherent Raman scattering . . . . . . . . . . . . . 12

2.2.1 Stimulated Raman scattering . . . . . . . . . . 12

2.2.2 Coherent anti-Stokes Raman scattering . . . . . . 13

2.2.3 CARS, SRS, and spontaneous Raman . . . . . . . 15

2.3 Broadband SRS . . . . . . . . . . . . . . . . 15

2.4 Broadband CARS . . . . . . . . . . . . . 16

2.5 Phase shaped broadband CARS . . . . . . . . . . . 18

2.5.1 Introduction . . . . . . . . . . . . . 18

$2.5 .2 \pi$ phase step . . . . . . . . . . . . . . . 19

2.5.3 2-Dimensional spectra . . . . . . . . . . . . 28

2.5.4 $2 \pi$ phase step . . . . . . . . . . . 30

3 Cross-polarized broadband SRS 31

3.1 Introduction . . . . . . . . . . . . . . . . 32

3.2 Setup . . . . . . . . . . . . . . . 32

3.2 .1 Working principle . . . . . . . . . . 32

3.2 .2 Overview . . . . . . . . . . . . . . 35 
3.2 .3 Laser synchronization . . . . . . . . . . . . . 37

3.3 Results and discussion . . . . . . . . . . . . . . . . 39

4 Numerical optimization of CARS 47

4.1 Introduction . . . . . . . . . . . . . . 48

4.2 The complex vibrational response . . . . . . . . . . . . 49

4.2.1 Modelling the vibrational response . . . . . . . . 49

4.2.2 Covariance matrix adaptation evolution strategy . 50

4.2.3 Optimization of the vibrational response model . . 51

4.2.4 Non-resonant background . . . . . . . . . . . . . 53

4.3 Simulating the CARS response . . . . . . . . . . . . 53

4.4 Single compound optimization . . . . . . . . . . . . 55

4.5 Selective excitation . . . . . . . . . . . . . 57

4.5.1 Multiple compound mixtures . . . . . . . . . . 57

4.5.2 Influence of homodyne mixing . . . . . . . . . . 61

4.5.3 Robustness to noise . . . . . . . . . . . . 62

4.5.4 Optimization dimensionality .......... . . 64

4.6 Solution landscapes . . . . . . . . . . . . . . . . 67

4.6 .1 Introduction . . . . . . . . . . . . . . 67

4.6.2 Optimization parameters . . . . . . . . . . 68

4.6.3 Optimization landscapes . . . . . . . . . . . . . . . . 69

4.6.4 Modified basis sets . . . . . . . . . . . . 77

5 Phase shaped CARS spectroscopy and imaging 81

5.1 Introduction . . . . . . . . . . . . . . 82

5.2 Setup . . . . . . . . . . . . . . . . 83

5.3 Sample materials . . . . . . . . . . . . . . . 86

5.4 Phase step profiles . . . . . . . . . . . . . . 86

$5.4 .1 \pi$ phase step . . . . . . . . . . 87

$5.4 .22 \pi$ phase step . . . . . . . . . . . . . . . . . 92

5.5 Complex profiles . . . . . . . . . . . . . 93

5.5.1 Single compound optimization . . . . . . . . . 94

5.5.2 Multiple compound mixtures . . . . . . . . . . 98

5.6 Discussion . . . . . . . . . . . . . . . . . 103

$\begin{array}{ll}\text { Appendix } & 105\end{array}$

$\begin{array}{ll}\text { A SLM calibration } & 105\end{array}$

A.1 SLM calibration . . . . . . . . . . . 106

A.1.1 Introduction . . . . . . . . . . . 106

A.1.2 Phase-only SLMs . . . . . . . . . . . . 106 
A.1.3 Phase and amplitude SLMs . . . . . . . . . . . . 109

A.2 SLM calibration verification . . . . . . . . . . . 117

A.2.1 Fundamental light . . . . . . . . . . . . . 117

A.2.2 Second harmonic generation . . . . . . . . . 117

A.2.3 Autocorrelation . . . . . . . . . . . . . . 118

A.2.4 Frequency resolved optical gating . . . . . . . . 120

$\begin{array}{ll}\text { B Calcite crystal mounts } & 123\end{array}$

$\begin{array}{lr}\text { Bibliography } & 125\end{array}$

$\begin{array}{ll}\text { Summary } & 133\end{array}$

$\begin{array}{ll}\text { Samenvatting } & 135\end{array}$

$\begin{array}{ll}\text { Dankwoord } & 137\end{array}$

$\begin{array}{ll}\text { List of publications } & 141\end{array}$ 


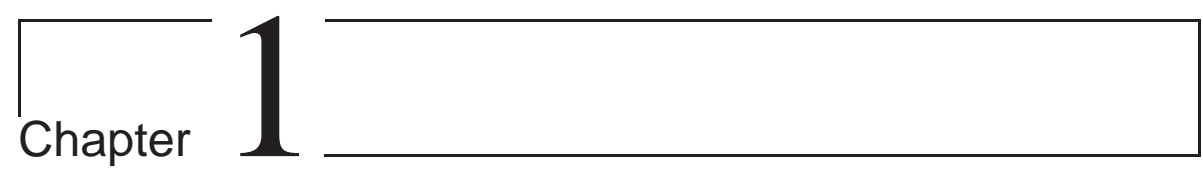

\section{Introduction}

\subsection{Optical microscopy}

Light and matter are two of the fundamental concepts in the universe which we experience daily. It is due to the (elastic) scattering of light by matter, first described by Lord Rayleigh [1,2] and Gustav Mie [3], that we can use our eyes to observe the world around us. When light and matter interact, their properties can be altered, and this allows us to learn more about either the light or the matter in question.

Advances in lens fabrication around the year 1600 paved the way for the first telescopes and microscopes to be constructed. These first optical imaging devices allowed the study of objects which are impossible to observe with the naked eye, such as the planets in our solar system and their satellites, or very small objects, such as cells and microbes. It was Antonie van Leeuwenhoek who significantly improved the microscope over the following decades, using a single lens design. He used his microscopes for biological imaging and some of the samples he observed include red blood cells, bacteria and human sperm.

The microscopes used by Antonie van Leeuwenhoek generated contrast based on differences in the absorption of light in the sample. It took until the twentieth century for microscopy techniques based on other contrast mechanisms to be developed. One of the first new optical microscopy techniques, developed around 1930, was fluorescence microscopy, in which the contrast is based on the presence of fluorescent molecules. Fluorescence had already been observed in the nineteenth 
century and was described by Sir George Stokes in 1852 [4], but it took until around 1930 for fluorescent molecules to be used for staining of biological samples.

To generate fluorescence, a photon is absorbed by a molecule, exciting the molecule to an electronic excited state. Some of the energy is lost non-radiatively as the molecule relaxes to the lowest level electronic excited state, after which a longer wavelength photon is emitted as the molecule decays back to the ground state. Fluorescent molecules can be engineered to bind to certain types of molecules, which can subsequently be imaged selectively. The ability to obtain selectivity based on the chemical properties of a substance is a valuable tool and one of the main reasons why fluorescence microscopy has become a widely used microscopy technique $[5,6,7]$. However, fluorescent marker molecules are often toxic and may influence the sample. Furthermore, fluorescence from other compounds in the sample will diminish the contrast.

Spontaneous Raman microscopy overcomes some of the disadvantages of fluorescence microscopy. It was developed around the same time as fluorescence microscopy, after the Raman effect had been observed by Sir Chandrasekhara Raman in 1928 [8]. In spontaneous Raman scattering, a photon interacts with the molecule and a lower wavelength photon is scattered as the molecule transitions to a vibrational state. The energy difference between the incident photon and the scattered photon is converted into a vibrational motion of the molecule and corresponds to the energy of a vibrational level of the molecule. Since the energies of the vibrational levels of a molecule depend on its chemical structure, this method provides chemical contrast. Spontaneous Raman scattering thus provides chemically selective imaging without the need for fluorescent markers. Only a very small fraction of incident photons is Raman scattered, leading to long acquisition times.

A few years after fluorescence microscopy and spontaneous Raman microscopy had started to become established tools, Frits Zernike introduced phase contrast microscopy [9], in which the contrast is based on phase differences in the transmitted light caused by differences in refractive index in the sample. To visualize the phase differences, Zernike combined the transmitted beam with a reference beam, leading to interference due to the phase differences accumulated in the sample. 

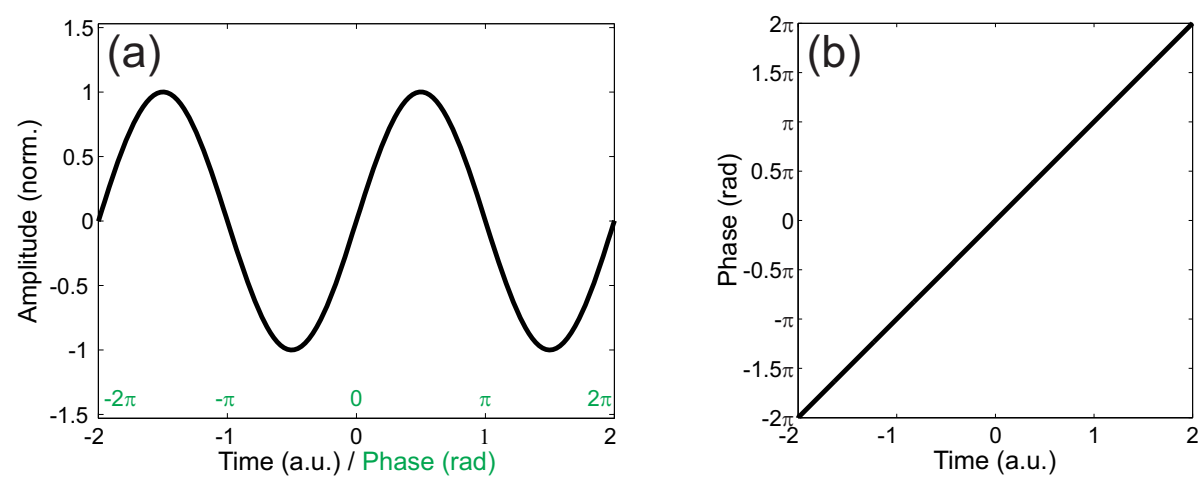

Figure 1.1: (a) Two periods of a sine wave, corresponding to $4 \pi$ phase. (b) The progression of the phase in (a) as a function of time.

\subsection{Phase and interference}

Phase and interference are two concepts which play a crucial role in Zernike's phase contrast microscopy, as well as the new microscopy techniques described in this thesis. They are best understood by considering light as an electromagnetic wave. We consider a simple sine wave, as shown in figure 1.1. Such a wave can be described with a number of parameters, such as the amplitude and wavelength (or frequency) of the wave. Another important parameter is the phase of the wave. The phase indicates the position in a cycle, going from 0 to $2 \pi$ over a full oscillation.

If we consider two waves of the same frequency, the phases will progress at the same rate. There can be a constant phase difference between the two waves, which can range from 0 to $2 \pi$. If these two waves are superimposed, the combination is equal to the algebraic sum of both waves [10]. The phase difference between the two waves determines whether there will be constructive interference ( 0 phase difference, figure 1.2(a)) or destructive interference ( $\pi$ phase difference, figure 1.2(b)) or anything in between. Hence, control over the phase difference between the two waves provides control over the amplitude of the combined wave.

Femtosecond modelocked laser pulses contain many different frequencies of light $\left( \pm 2 * 10^{5}\right.$ in our case). These different frequencies have a fixed frequency-phase relation, leading to a pulsed output. The phase of the different frequencies progresses at different rates. There can also be a phase offset with respect to the envelope of the laser pulse for the different frequencies, which determines when the different frequen- 

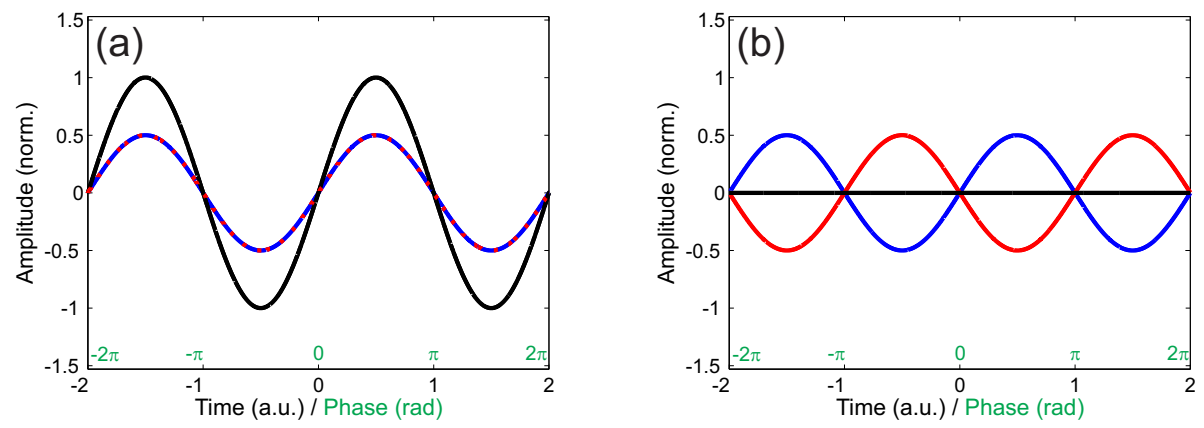

Figure 1.2: (a) Two sine waves (red and blue, dashed) with 0 phase difference undergo constructive interference. The result is a wave with double the amplitude (black). (b) Two sine waves (red and blue) with a $\pi$ phase difference undergo destructive interference. The two waves cancel each other out completely and the result is zero (black).

cies interfere constructively or destructively. If this phase difference between the different frequencies is zero, a very short pulse, also called a transform-limited or Fourier-limited pulse, is produced (figure 1.3(a,c)). By changing the phase differences between the different frequencies, the shape of the laser pulse can be changed, as shown in figure 1.3(b,d). Using the phase to control the laser pulse and the phase of generated light forms the basis of the microscopy techniques described in this thesis.

\subsection{Nonlinear microscopy}

The invention of the laser by Theodore Maiman in 1960 [11] provided high intensity, collimated, coherent light, opening the door for the development of nonlinear microscopy techniques, which are based on the simultaneous absorption of multiple photons. Many nonlinear microscopy techniques were developed to provide contrast based on various sample properties and/or to circumvent limitations of the linear microscopy techniques. An advantage of nonlinear microscopy techniques is that they are inherently confocal, due to their nonlinear intensity dependence. A confocal microscope has increased depth-resolution due to the reduction in contributions from regions of the sample that are (axially) out of focus.

The lowest order nonlinear microscopy techniques require two photons and include second harmonic generation and two-photon fluorescence. Second harmonic generation microscopy provides contrast based on the 

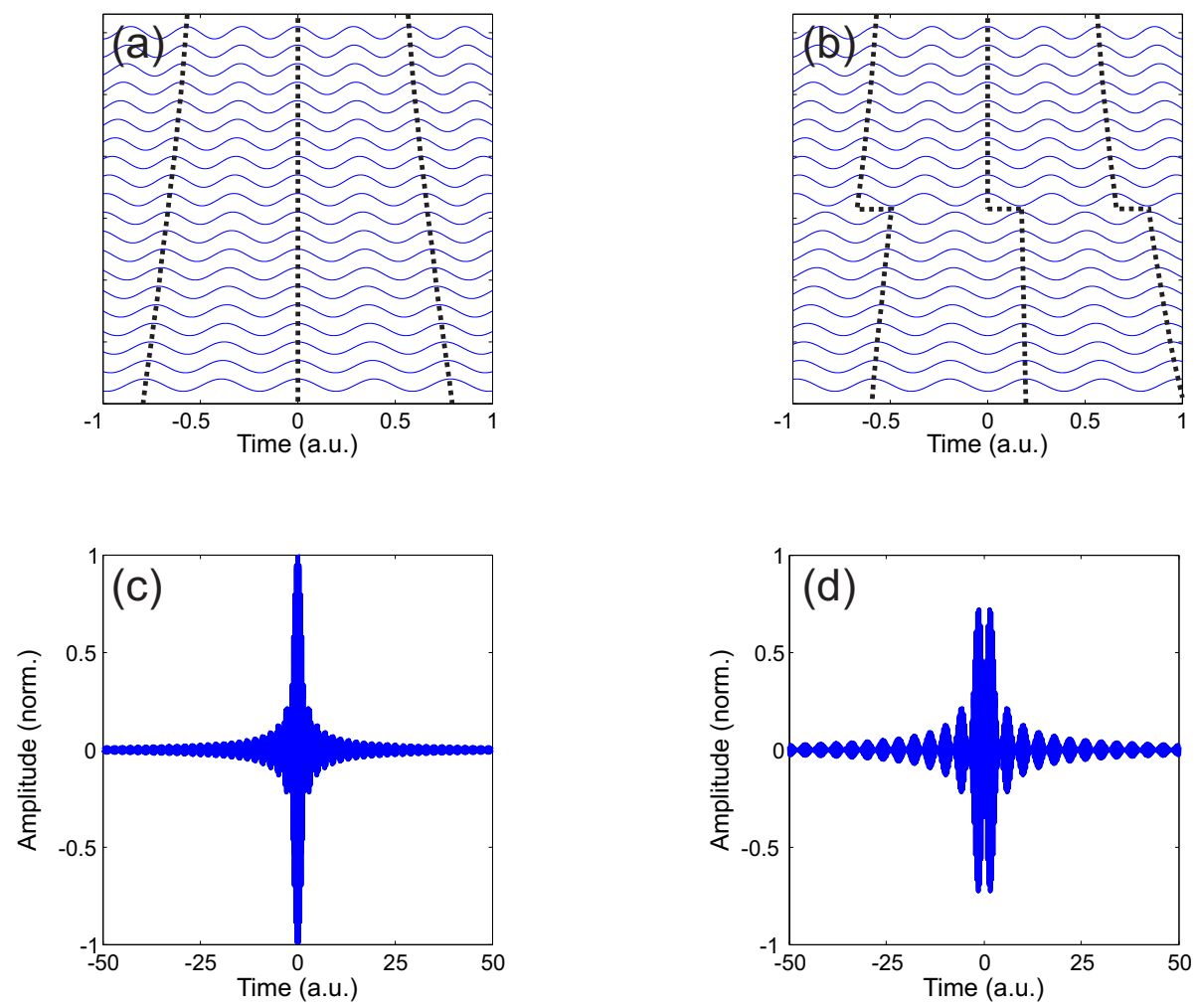

Figure 1.3: (a,b) Twenty waves with different (linearly spaced) frequencies, the relative phase is indicated by the dashed black lines. (c,d) The pulses resulting from the summation of two hundred waves around the same center frequency as the waves in $(a, b)$. (a,c) All the waves are in phase, producing a transform-limited pulse. $(b, d)$ The high frequencies have a $\pi$ phase offset compared to the low frequencies, producing a double pulse. 
second order nonlinear susceptibility of the sample and is often used for the imaging of collagen. The second order nonlinear susceptibility requires non-centrosymmetry in the molecular structure, which is effectively negligible in most materials.

In two-photon fluorescence [12], a fluorescent molecule is excited by the simultaneous absorption of two photons. The energy required to excite the fluorescent molecule is now provided by two photons instead of one photon, so typically lower frequency (near infrared) light is used.

Third order nonlinear microscopy techniques include third harmonic generation [13], which reveals interfaces and inhomogeneities in samples, and several Raman-based techniques. Nonlinear Raman techniques provide contrast based on the vibrational response of the sample, similar to spontaneous Raman scattering. In this thesis I will focus on two nonlinear Raman techniques, namely stimulated Raman scattering (SRS) and coherent anti-Stokes Raman scattering (CARS), which coherently stimulate the Raman process, leading to chemically selective detection with faster acquisition times. CARS and SRS have generated a lot of interest over the last decades for a variety of applications, including gas-phase thermometry $[14,15,16,17]$ and biomedical imaging $[18,19,20,21,22]$.

\subsection{Thesis overview}

This thesis describes new broadband techniques for coherent Raman scattering (CRS). These broadband CRS techniques are able to obtain chemically selective information from a sample by probing multiple vibrational resonances simultaneously. A broadband stimulated Raman scattering (SRS) approach, based on a common-path interferometer, is introduced as well as a broadband coherent anti-Stokes Raman scattering (CARS) approach. Our broadband CARS approach uses spectral phase shaping to influence the interference between different pathways that lead to the same generated CARS wavelength.

With our broadband CRS techniques we aim to create powerful imaging techniques for use in biomedical imaging, high throughput screening, and novel material characterization. Ideally, such a technique provides high contrast non-invasive imaging with low acquisition times and a high degree of sensitivity and chemical selectivity, whilst being free of inter- 
fering background signals. Preferably, such a technique is low cost and easy to use to facilitate widespread use. The CRS techniques presented in this thesis resolve some of the drawbacks of conventional CRS implementations and represent a big step forward towards the goal of such an ideal imaging technique.

Chapter 2 contains the theory behind narrowband and broadband SRS and CARS. Furthermore, the advantages of spectral phase shaping for broadband CARS are explained and the possibility of non-resonant background suppression and chemically selective imaging are treated theoretically, using an intuitive shaping strategy based on $\pi$ and $2 \pi$ phase steps.

Chapter 3 presents a new broadband SRS approach, based on common-path interferometry. In this common-path interferometer, orthogonal polarization states are used to generate SRS in one polarization, whilst the other polarization is used as a reference. Our broadband SRS approach provides modulation free, spectrally resolved detection of the SRS signal. The results of this technique are presented as well as suggestions on improving this technique.

Chapter 4 covers a numerical study on optimizing the spectral phase for broadband CARS to obtain high contrast chemically selective imaging. Numerical optimization of the phase is performed using an adaptive algorithm known as covariance matrix adaptation evolution strategy (CMA-ES). The robustness of this optimization approach to homodyne mixing and phase noise is treated. Furthermore, a study on improving the shape of the optimization landscape around the optimum, by modifying the basis set of parameters that describe the phase function, is presented.

Chapter 5 contains the results of phase shaped broadband CARS experiments. The results for the shaping strategies based on $\pi$ and $2 \pi$ phase steps, as outlined in chapter 2, are presented. Furthermore, chemically selective imaging based on experimental optimization of the phase profile using CMA-ES is shown, including selective imaging in samples containing up to five resonant compounds. 
Chapter 1 


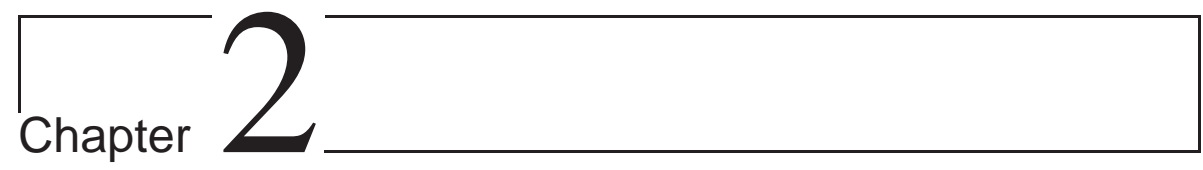

\section{Broadband coherent Raman scattering}

Raman interactions occur between light and the vibrational resonances within a molecule. This chapter introduces the Raman effect and covers two coherently stimulated Raman processes, namely stimulated Raman scattering (SRS) and coherent anti-Stokes Raman scattering (CARS). The more conventional narrowband implementations of CARS and SRS are covered, as well as various extensions of these techniques using broadband excitation. Our broadband CARS approach is explained in detail, as well as the influence that spectral phase shaping of the excitation pulses has on the CARS signal and how it can be used to suppress the non-resonant background and obtain chemically selective imaging. 


\subsection{Interaction of light and matter}

\subsubsection{Introduction}

Light can be described as an electromagnetic wave, that interacts with the electrons surrounding atoms. In molecules, we can describe this interaction by the polarizability. In linear optics the polarizability is linearly related to the field strength and can be described as shown in equation $2.1[23]$.

$$
\tilde{P}(t)=\chi^{(1)} \tilde{E}(t)
$$

where $\tilde{P}(t)$ is the linear polarizability, $\tilde{E}(t)$ is the time dependent electric field of the incident light wave, and $\chi^{(1)}$ is a material dependent property, known as the linear susceptibility. $\chi^{(1)}$ is a tensor of rank 2, but in this case we limit the discussion to the $\chi_{11}^{(1)}$ tensor element and describe $\chi^{(1)}$ as a constant.

There are also higher order contributions to the polarizability however, that depend nonlinearly on the incident field strength. These higher order contributions can be included by expanding the polarizability as a power series with respect to the field strength $\tilde{E}(t)$, as shown in equation 2.2 .

$$
\begin{array}{r}
\tilde{P}(t)=\chi^{(1)} \tilde{E}(t)+\chi^{(2)} \tilde{E}^{2}(t)+\chi^{(3)} \tilde{E}^{3}(t)+\ldots \\
\equiv \tilde{P}^{(1)}(t)+\tilde{P}^{(2)}(t)+\tilde{P}^{(3)}(t)+\ldots
\end{array}
$$

where $\tilde{P}(t)$ is the total polarizability, $\chi^{(1)}$ is the linear susceptibility of the molecule, $\chi^{(2)}$ and $\chi^{(3)}$ are the second and third order nonlinear susceptibility respectively, and $\tilde{E}(t)$ is the field strength of the incident lightwave.

The polarizability can subsequently be separated into the different orders of contributing terms, as shown in equation 2.3. The nonlinear susceptibilities $\left(\chi^{(2)}, \chi^{(3)}, \cdots\right)$ decrease rapidly with increasing order and only become relevant when a high intensity light source is used. Since the invention of the laser [11], which provides high intensity coherent light, these effects have become more readily observable.

The first term, $\tilde{P}^{(1)}$, is the linear polarizability, as in equation 2.1. The second term, $\tilde{P}^{(2)}$, is the second order nonlinear polarizability, which scales quadratically with the field strength. This term is zero in most 
cases, except in non-centrosymmetric environments, such as in certain crystals or at the interface between two different materials. Second order nonlinear optical effects, such as second harmonic generation and sum-frequency generation, occur only when $\tilde{P}^{(2)}$ is non-zero.

The third order nonlinear polarizability, $\tilde{P}^{(3)}$, is the next lowest order nonlinear contribution to the polarization. Contrary to $\tilde{P}^{(2)}, \tilde{P}^{(3)}$ is significant in almost all materials. The third order nonlinear polarizability gives rise to a large number of nonlinear effects, including third harmonic generation, the optical Kerr effect, cross-phase modulation, and four-wave mixing. An interesting subset of the third order nonlinear optical effects is the set of Raman-based effects. These effects rely on the interaction between photons and the vibrational motion of a molecule.

\subsubsection{Spontaneous Raman scattering}

The spontaneous Raman effect was discovered by C.V. Raman in 1928 [8] and can be observed by illuminating a sample with monochromatic light and collecting the scattered light. If the collected light is resolved spectrally, light of a lower frequency than the incident light can be detected. This lower frequency light is generated due to an interaction between the incident light and the molecules in the sample. If a photon is scattered by the molecule, its frequency will generally remain unaltered (Rayleigh scattering). A small amount of photons will scatter inelastically, in which case the remaining energy from the absorbed photon is converted into a vibrational motion of the molecule. This is called (spontaneous) Raman scattering.

We can describe the vibrational response of a molecule using a massspring system as a simplified model of the induced dipole. Each vibrational resonance has a specific frequency, that depends on the polarizability of the molecule. This polarizability is affected by the presence of nuclear normal modes [24]. Heavy atoms and weak bonds will generally result in a lower frequency of vibration, while light atoms and strong bonds will result in a higher frequency of vibration. The vibrational frequency can also be influenced by neighbouring (groups of) atoms or molecules [25].

In a time-invariant model, where there is no cross-coupling between 
resonances, we can model the vibrational response of a molecule as a sum of individual harmonic oscillator responses with different amplitudes $\left(A_{R}\right)$, resonance frequencies $\left(\omega_{R}\right)$ and linewidths $\left(\gamma_{R}\right)$. The spontaneous Raman scattering signal is proportional to the imaginary part of this vibrational response, as shown in equation 2.4.

$$
I_{\text {Raman }} \propto \Im\left(\chi^{(3)}\right)=\Im\left(\sum_{R} \frac{A_{R}}{\omega_{R}^{2}-\omega^{2}-i \gamma_{R} \omega}\right)=\sum_{R} \frac{A_{R} \gamma_{R} \omega}{\left(\omega_{R}^{2}-\omega^{2}\right)^{2}+\gamma_{R}^{2} \omega^{2}}
$$

The spontaneous Raman scattering signal is generated at Stokesshifted frequencies, $\omega_{s}$, which are determined by the frequencies of the vibrational resonances of the molecule $\left(\omega_{R}\right)$ and the excitation (pump) frequency $\left(\omega_{p}\right)$, as shown in equation 2.5 .

$$
\omega_{s}=\omega_{p}-\omega_{R}
$$

\subsection{Coherent Raman scattering}

Spontaneous Raman scattering (figure 2.1(a)) is very useful for collecting vibrational information from a sample, and the entire vibrational spectrum can be obtained in a single measurement. Due to the low scattering cross section, only a small fraction of the incident light is Raman scattered. Instead of collecting the spontaneous Raman scattering, the scattering process can be stimulated coherently. In sections 2.2.1 and 2.2.2 two coherently stimulated Raman processes, namely stimulated Raman scattering and coherent anti-Stokes Raman scattering, are described. These two stimulated Raman processes have generated a lot of interest over the last decades for a variety of applications, including biomedical imaging $[18,19,20,21,22]$.

\subsubsection{Stimulated Raman scattering}

In stimulated Raman scattering (SRS) [26] two coherent light sources of different wavelengths are used. The lower wavelength light is labeled the 'pump' and the higher wavelength light is labeled the 'Stokes'. If the frequency difference between the pump and the Stokes matches the 


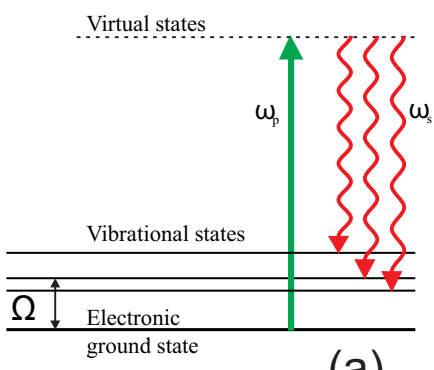

(a)

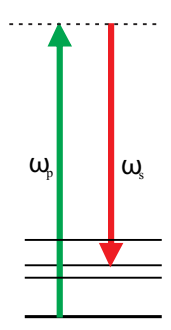

(b)

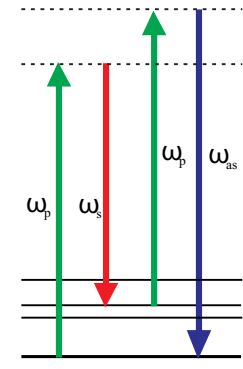

(c)



(d)

Figure 2.1: Energy diagrams for (a) spontaneous Raman scattering, (b) stimulated Raman scattering, (c) coherent anti-Stokes Raman scattering, and (d) non-resonant four-wave mixing.

frequency of a vibrational resonance in the molecule, there will be stimulated population transfer from the ground state to the vibrational state. This process occurs via the absorption of a pump photon, and a stimulated emission at the Stokes wavelength, as shown in figure 2.1(b).

The increase in Raman scattered light when the frequency difference between the pump and the Stokes matches with a vibrational frequency, manifests itself as a loss of pump photons and a gain of Stokes photons. Even though the Raman scattering is stimulated, the loss on the pump beam and the gain on the Stokes beam are still small $\left(<10^{-4}\right)$. Detecting this change in intensity is challenging, but can be accomplished by high frequency modulation of one of the beams and lock-in detection of the other beam $[20,27]$.

\subsubsection{Coherent anti-Stokes Raman scattering}

Another coherent Raman scheme that has found widespread use is coherent anti-Stokes Raman scattering (CARS) [18, 28], of which the energy diagram is shown in figure 2.1(c). In CARS, three input fields combine to form an anti-Stokes shifted output field, as shown in equation 2.6. 


$$
\begin{gathered}
\omega_{a s}=\omega_{\text {pump }}-\omega_{\text {Stokes }}+\omega_{\text {probe }} \\
k_{\text {as }}=k_{\text {pump }}-k_{\text {Stokes }}+k_{\text {probe }}
\end{gathered}
$$

The anti-Stokes signal is blue-shifted with respect to the input wavelengths and can be detected by spectral filtering, providing easier detection compared to SRS. Since the output field is blue-shifted from the input fields, CARS is inherently free from one-photon fluorescence. The anti-Stokes output is resonantly enhanced when the difference in frequency between the pump and the Stokes matches with a vibrational resonance of the molecule. Since CARS is a parametric process there is also conservation of momentum, as shown in equation 2.7. The conservation of momentum means the output field is directional and that it can be easily collected using a microscopy objective, facilitating easier detection compared to spontaneous Raman scattering. The pump and probe frequencies are generally chosen to be degenerate, since this reduces the required number of coherent light sources to two. Most CARS implementations use near-infrared excitation light to avoid electronic excitation in the sample and limit photothermal damage [18].

Alongside the CARS process, another four-wave mixing process occurs, as shown in figure 2.1(d), that also generates a signal at the antiStokes wavelength. This four-wave mixing process generates signal even in the absence of any vibrational resonances, which means that it constitutes an unwanted non-resonant background in the CARS signal. This non-resonant background has a flat phase response. Furthermore, there is homodyne mixing between the resonant and non-resonant contributions to the CARS signal, as shown in equation 2.8 .

$$
\begin{aligned}
I_{C A R S}(\omega) & \propto\left|\chi^{(3)}(\omega)\right|^{2} \\
& =\underbrace{\left|\chi_{R}^{(3)}(\omega)\right|^{2}}_{(\text {Resonant })}+\underbrace{\left|\chi_{N R}^{(3)}\right|^{2}}_{(\text {Non-Resonant })}+\underbrace{2 \chi_{N R}^{(3)} \operatorname{Re}\left[\chi_{R}^{(3)}(\omega)\right]}_{(\text {Mixing })}(2.8)
\end{aligned}
$$

Many techniques have been developed to suppress or remove this non-resonant background, including heterodyning [29, 30], spectral phase and polarization shaping [31, 32], time-delayed detection [33, 34], frequency modulation [35], and polarization-based detection [36, 37]. 


\subsubsection{CARS, SRS, and spontaneous Raman}

CARS and SRS provide faster detection compared to spontaneous Raman scattering, due to the increase in generated signal. SRS and CARS have a nonlinear intensity dependence, opposed to the linear intensity dependence of spontaneous Raman scattering. Spontaneous Raman scattering and stimulated Raman scattering have a linear concentration dependence (equations 2.9 and 2.10). The dependence of the CARS signal on the concentration is not trivial however, due to the homodyne mixing with the non-resonant background (equation 2.8). In the case of strong resonant scattering, the $\left|\chi_{R}^{(3)}\right|^{2}$ term dominates and the CARS signal depends quadratically on the concentration. In the case of weak scattering and/or a strong non-resonant background, the homodyne mixing term, $2 \chi_{N R}^{(3)} \operatorname{Re}\left[\chi_{R}^{(3)}(\omega)\right]$, dominates and the CARS signal will depend linearly on the concentration (equation 2.11) [38].

$$
\begin{array}{r}
I_{\text {Raman }} \propto N * I_{\text {pump }} \\
I_{S R S} \propto N * I_{\text {pump }} * I_{\text {Stokes }} \\
I_{C A R S} \propto N^{\alpha} * I_{\text {pump }} * I_{\text {Stokes }} * I_{\text {probe }}, 1 \leq \alpha \leq 2
\end{array}
$$

where $\mathrm{N}$ is the number of oscillators, $\alpha$ is a constant, and I denotes the intensities of the various input and output beams.

The signal from SRS and CARS is generally much stronger than the spontaneous Raman scattering signal, which is critical for fast (realtime) imaging. The main disadvantage however is that the CARS and SRS techniques we have considered here only provide information based on a single vibrational resonance at a time, where spontaneous Raman scattering yields information on the full vibrational spectrum. One would like to combine the speed of SRS and CARS with the ability to obtain information based on multiple resonances such as in spontaneous Raman scattering. Sections 2.3 and 2.4 focus on broadband SRS and broadband CARS respectively, where multiple resonances are excited simultaneously using femtosecond laser pulses.

\section{$2.3 \quad$ Broadband SRS}

To increase the selectivity and specificity, one would like to obtain information based on multiple resonances, which can be excited simul- 
taneously using broadband excitation. With a broadband pump and a narrowband Stokes, or a broadband Stokes and a narrowband pump, each frequency in the broadband pulse generates a unique difference frequency in combination with the Stokes. The narrowband pulse effectively projects the complete bandwidth of the broadband pulse onto the frequency region of the vibrational resonances. Every resonance frequency thus corresponds to a single frequency in the broadband pulse and therefore every resonance shows up as a loss or a gain at a specific frequency in the broadband pulse.

To detect the frequencies of the different vibrational resonances, the loss or gain in the broadband pulse has to be detected in a spectrally resolved manner. Spectrally resolved detection is possible but requires careful consideration of the choice of detector [39]. Similar to narrowband SRS, lock-in based detection could be used to improve the signalto-noise ratio of the detected signal. Spectrally resolved lock-in amplified detection is not trivial however and has to our knowledge not been reported for SRS to this date. A broadband SRS technique based on a single phase shaped broadband pulse has been reported by Silberberg et al. [40].

In chapter 3 a new broadband SRS approach based on a polarization based common-path interferometer is presented. Our SRS approach relies on a common-path interferometer, using polarization selection to reject most of the excitation background, and does not require modulation or lock-in amplified detection.

\subsection{Broadband CARS}

Instead of using narrowband pulses for CARS to excite a single resonance, as described in section 2.2.2, it is possible to use one or more broadband pulses instead. By using one or more broadband excitation pulses, multiple vibrational resonances can be accessed simultaneously. Several broadband CARS schemes have been developed, using different combinations of narrowband and broadband pulses.

In multiplex CARS [41, 42, 43, 44], a broadband pulse is used as Stokes, while the pump and probe are narrowband, as shown in figure 2.2(a). The combination of a narrowband pump and broadband 


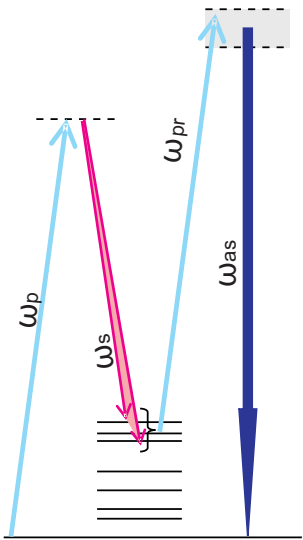

(a)

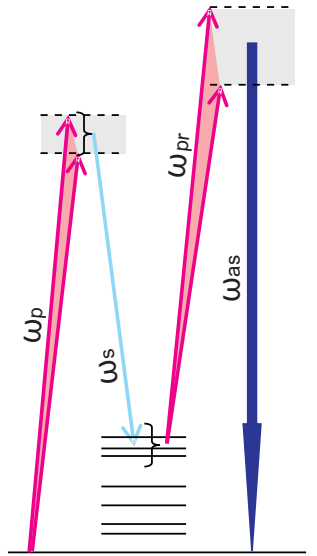

(b)

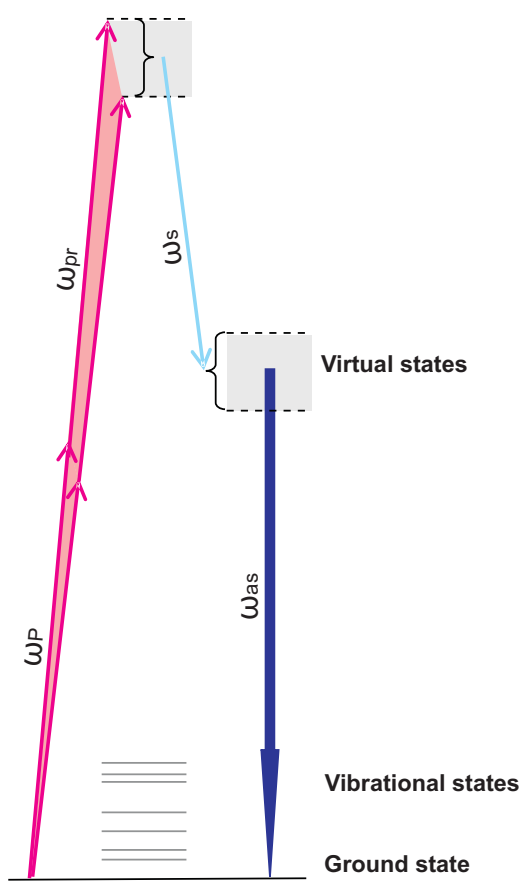

(c)

Figure 2.2: Energy diagrams for (a) multiplex CARS, (b) broadband pump and probe CARS, and (c) non-resonant four-wave mixing with a degenerate broadband pump and probe.

Stokes excites a broad band of vibrational resonances. The narrowband probe ensures a narrowband anti-Stokes shifted CARS signal from every resonance that is probed. These resonances show up on a nonresonant background signal that is generated over the full bandwidth of the broadband pulse. Several techniques have been reported to retrieve the resonant signal and remove the non-resonant background from such a spectrum $[45,46,47]$.

In single pulse CARS [31], the pump, Stokes, and probe are all obtained from a single broadband pulse. Different frequency pairs in the broadband pulse can combine to form the same difference frequency. By adjusting the relative phase between these frequency pairs, their interference at the difference frequency can be influenced. For higher difference frequencies, fewer and fewer frequency pairs contribute and the resolution progressively degrades. 
In this thesis a broadband CARS approach based on a degenerate broadband pump and probe pulse in combination with a narrowband Stokes pulse is presented (figure 2.2(b)). In our approach, the combination of a broadband pump and a narrowband Stokes excites a broad band of vibrational resonances, which are probed by the (degenerate) broadband probe pulse, providing a near constant resolution. There are different combinations of pump and probe frequencies, which result in the same anti-Stokes frequency, but have a different intermediate state near or on the vibrational states. This leads to interference in the CARS signal caused by the different pathways. The signal from a vibrational resonance will be distributed over a broad bandwidth by the broadband probe pulse, meaning that the resonances are not directly apparent in the detected CARS signal. We apply spectral phase shaping of our broadband pump and probe pulse to influence the interferences in the CARS signal, facilitating chemically selective imaging based on multiple resonances. Our phase shaping approach simultaneously removes the purely non-resonant background contributions (figure 2.2(c)). A mixing term between the resonant and non-resonant term remains (see equation 2.8).

\subsection{Phase shaped broadband CARS}

\subsubsection{Introduction}

Since CARS is a coherent process, the generated CARS field is influenced by the spectral phase of the input fields. By controlling the spectral phase of the input fields, the CARS process can be influenced. Using phase shaped broadband pulses, selective excitation of Raman levels [48, 49, 50], improved resolution [51], and nonresonant background rejection $[32,52]$ have been reported.

If we consider a time-invariant model, we can describe the CARS signal that is generated by convolutions of our input fields, multiplied by the vibrational response of the molecule, as shown in equation 2.12 .

$$
I_{C A R S}=\left|E_{a s}\right|^{2} \propto\left|\left(\left(E_{p} \otimes E_{S}\right) \cdot \chi^{(3)}\right) \otimes E_{p r}\right|^{2},
$$

In the case of a narrowband excitation pulse, the convolution can be approximated by a multiplication in the frequency domain, which amounts to a frequency shift. For a narrowband Stokes and a broadband 
degenerate pump and probe, equation 2.12 can be simplified to equation 2.13 .

$$
\begin{array}{r}
I_{C A R S} \propto\left|\left(\left|E_{p}\left(\omega+\omega_{s}\right)\right| e^{i \phi_{p}\left(\omega+\omega_{s}\right)} \cdot \chi^{(3)}(\omega)\right) \otimes\right| E_{p}(\omega)\left|e^{i \phi_{p}(\omega)}\right|^{2}, \\
\chi^{(3)}=\chi_{N R}^{(3)}+\chi_{R}^{(3)}=\chi_{N R}^{(3)}+\sum_{R} \frac{A_{R}}{\omega_{R}^{2}-\omega^{2}-i \gamma_{R} \omega}
\end{array}
$$

In section 2.5.2 phase shaping strategies for our CARS approach, using a narrowband Stokes and a degenerate pump and probe, are introduced. These shaping strategies are based around $\pi$ and $2 \pi$ phase steps and are used for spectroscopy, chemically selective imaging, and suppression of the non-resonant background contribution. Phase shaping strategies using more complex phase profiles are presented in chapters 4 and 5 .

\subsection{2 $\pi$ phase step}

A vibrational resonance is accompanied by a $\pi$ phase shift over the resonance. This phase shift will influence the broadband CARS response that is generated. By applying a spectral phase to the broadband pump and probe pulse, the CARS process can be influenced even further. We use an intuitive shaping strategy based on the $\pi$ phase step introduced by a vibrational resonance. By applying a similar, but inverted step to the broadband pulse, the phase step introduced by the vibrational resonance will be compensated. We label the applied $\pi$ phase step as a 'positive' $\pi$ phase step. As an effect of this positive $\pi$ phase step, destructive interference in the resonant CARS signal is minimized, which causes an increase in overall amplitude.

To visualize the effect of applying a $\pi$ phase step to the pump and probe pulse we simulate the CARS signal generated by a single resonance. We choose a broadband pump and probe pulse with a center wavelength of $806.7 \mathrm{~nm}\left(12396 \mathrm{~cm}^{-1}\right)$ and a full width at half maximum (FWHM) bandwidth of $16.3 \mathrm{~nm}\left(250 \mathrm{~cm}^{-1}\right)$. The Stokes pulse is approximated by a Dirac delta function at $1064.3 \mathrm{~nm}\left(9396 \mathrm{~cm}^{-1}\right)$. A single resonance is simulated with a center frequency of $3000 \mathrm{~cm}^{-1}$ $(89.94 \mathrm{THz})$, and a FWHM bandwidth of $10 \mathrm{~cm}^{-1}(0.3 \mathrm{THz})$. In figure 2.3 the effect of applying a compensating phase step to the pump and probe pulse is shown. The resonance frequency corresponds to a pump frequency that is close to the center frequency of the pump pulse (see 

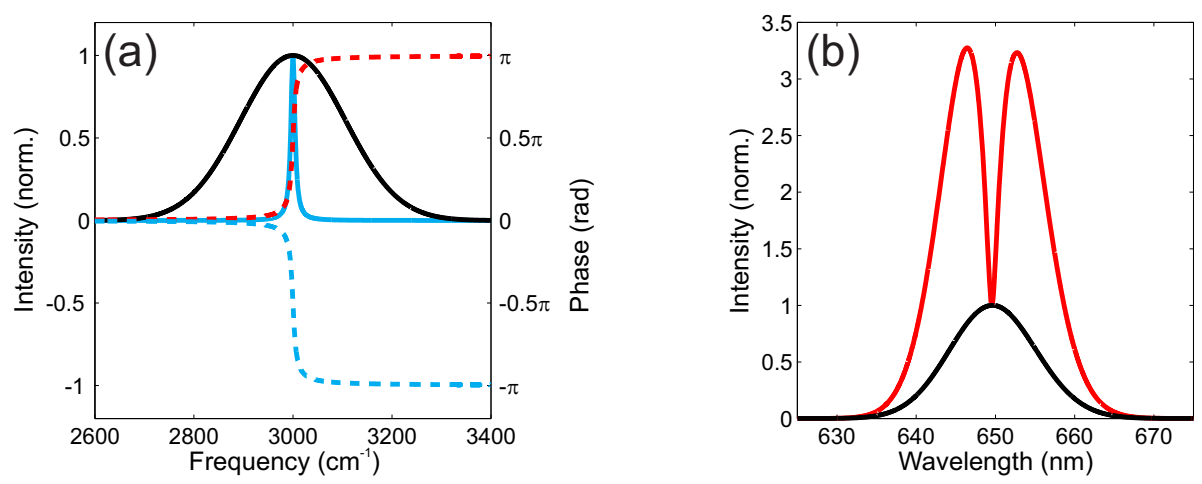

Figure 2.3: (a) $-\Im\left(\chi^{(3)}\right)$ (cyan solid) and phase (cyan dashed) of the vibrational resonance. Furthermore, the pump and probe pulse intensity (black) and phase (red), shifted by the Stokes frequency, are shown. (b) CARS spectrum for an unshaped pump and probe pulse (black) and for a pump and probe pulse with a positive $\pi$ phase step (red).

figure 2.3(a)). Figure 2.3 shows a large increase in CARS signal due to the applied phase step.

In figure 2.3 only the resonant contribution to the CARS signal is shown. There is also a non-resonant contribution, that interferes with the resonant contribution, as shown in figure 2.4. The total amount of signal depends on the amount of non-resonant background. Here, we choose a non-resonant background of $20 \%$ (peak to baseline).

Figure 2.4(b) shows a dip in the resonant contribution at the location of the phase step. The non-resonant contribution has a peak at the location of the phase step. Even though the resonant signal is enhanced by the phase shaping, there is still a substantial non-resonant contribution to the CARS signal as well.

It should be noted that the non-resonant contribution is unaffected by the vibrational resonance, and has a flat spectral phase response. The non-resonant contribution is therefore insensitive to the sign of the phase profile that is applied to the pump and probe pulse. Applying the inverse phase profile will thus generate the same non-resonant signal. By subtracting two spectra obtained with inverse phase profiles from each other, a difference signal which is free of the purely non-resonant background can be obtained. The homodyne mixing between the resonant and non-resonant contribution will still be present in this difference 

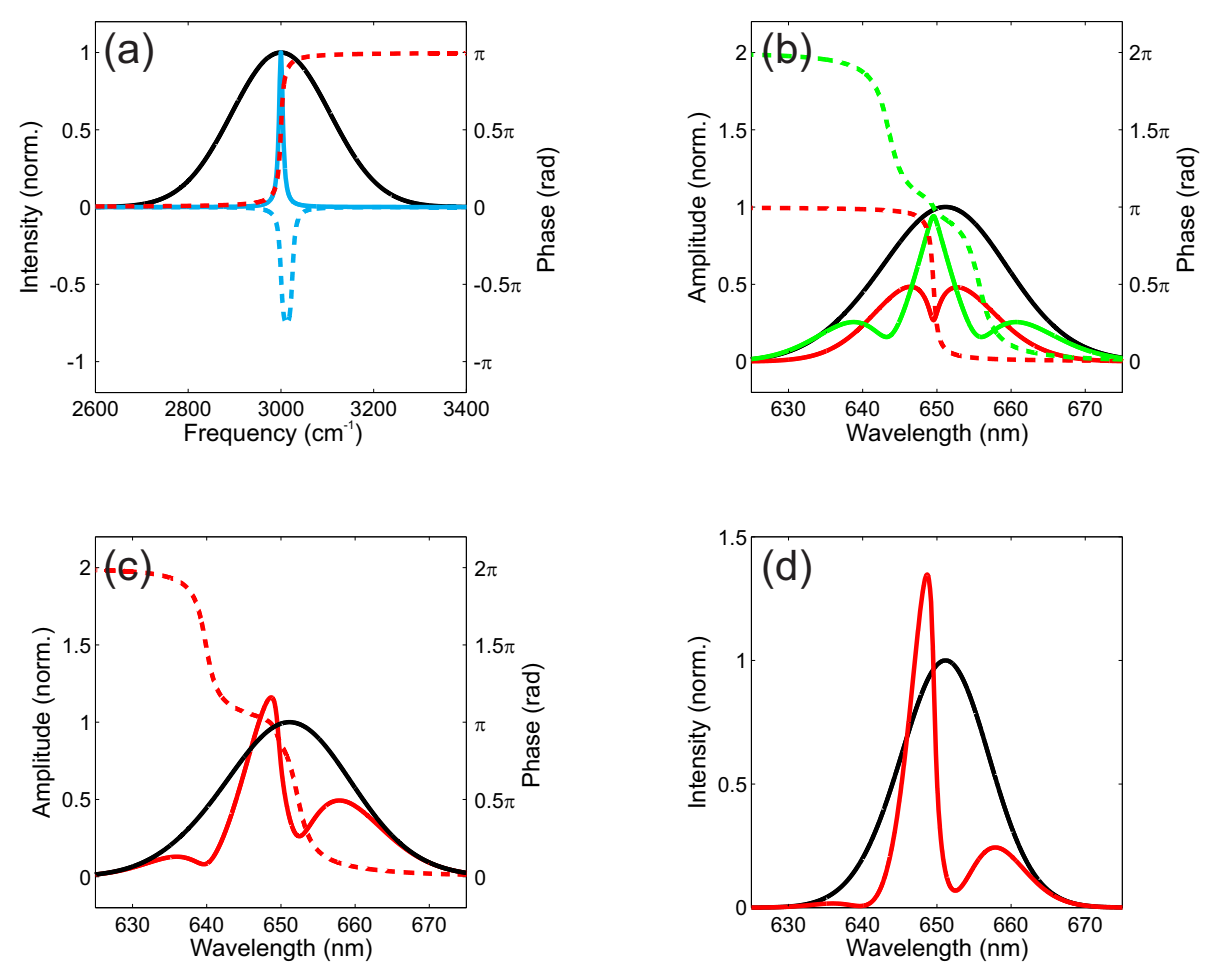

Figure 2.4: CARS response for a positive $\pi$ phase step. (a) $-\Im\left(\chi^{(3)}\right)$ (cyan solid) and phase (cyan dashed) of the vibrational resonance. Furthermore, the pump and probe pulse intensity (black) and phase (red), shifted by the Stokes frequency, are shown. (b) Resonant (red) and nonresonant (green) amplitude (solid) and phase (dashed) of the CARS signal for a pump and probe pulse with a positive $\pi$ phase step. The CARS amplitude for an unshaped pump and probe pulse (black) is shown for comparison. (c) Total CARS amplitude (red solid) and phase (red dashed) for a pump and probe pulse with a positive $\pi$ phase step. The CARS amplitude for an unshaped pump and probe pulse (black) is shown for comparison. (d) Total CARS intensity for a pump and probe pulse with a positive $\pi$ phase step (red) and for an unshaped pump and probe pulse (black). 
signal however. Because the mixing term also depends on the resonant contribution (equation 2.8), it does not cancel out in the subtraction.

By reversing the direction of the phase step, a phase step identical to the phase step of the molecular resonance is applied to the laser pulse. We label this step as a 'negative' $\pi$ phase step. In the case of this negative $\pi$ phase step, when the phase step of the pump pulse overlaps with the phase step associated with the molecular resonance, a combined phase step of $2 \pi$ is produced. This combined phase profile is similar to a flat phase profile, except for a region equal in size to the linewidth of the molecular resonance, where there is a slope in the phase profile. The $2 \pi$ slope acts as a differentiator in the convolution with the probe pulse. So there will be a sharply peaked response when the phase step of the probe pulse passes this $2 \pi$ phase step during the convolution. The CARS signal for this inverted phase step, when centered on the vibrational resonance, can be found in figure 2.5.

Comparing the results for a negative phase step (figure 2.5) to the results for a positive phase step (figure 2.4) reveals that the non-resonant contribution remains unchanged, because the sign of the phase step is irrelevant for the non-resonant contribution as it unaffected by the molecular resonance. Hence, we can remove the non-resonant background by subtracting the signals obtained for the positive and negative phase step from each other, as shown in figure 2.6. Figure 2.6(b) shows the purely non-resonant background free spectrum, revealing a dispersive lineshape caused by the homodyne mixing term (equation 2.8).

In the case of a negative phase step applied at the position of the resonance, the resonant and non-resonant contributions have a peak in the spectrum at the frequency that corresponds to the molecular resonance (figure 2.5(b)). By moving the phase step off the center of the resonance, a displacement of these features caused by the phase step is expected.

The non-resonant peak feature is caused by the convolution of the pump pulse and the probe pulse, which both have the same displaced phase step. In the convolution of these two pulses, the point where these phase steps cross is thus displaced twice as far. For a scanning $\pi$ phase step this creates a feature that moves at twice the speed of this scanning phase step. The effect of a displaced phase step on the non-resonant CARS signal is shown in figure 2.7(c,f). In this case the small deviation 

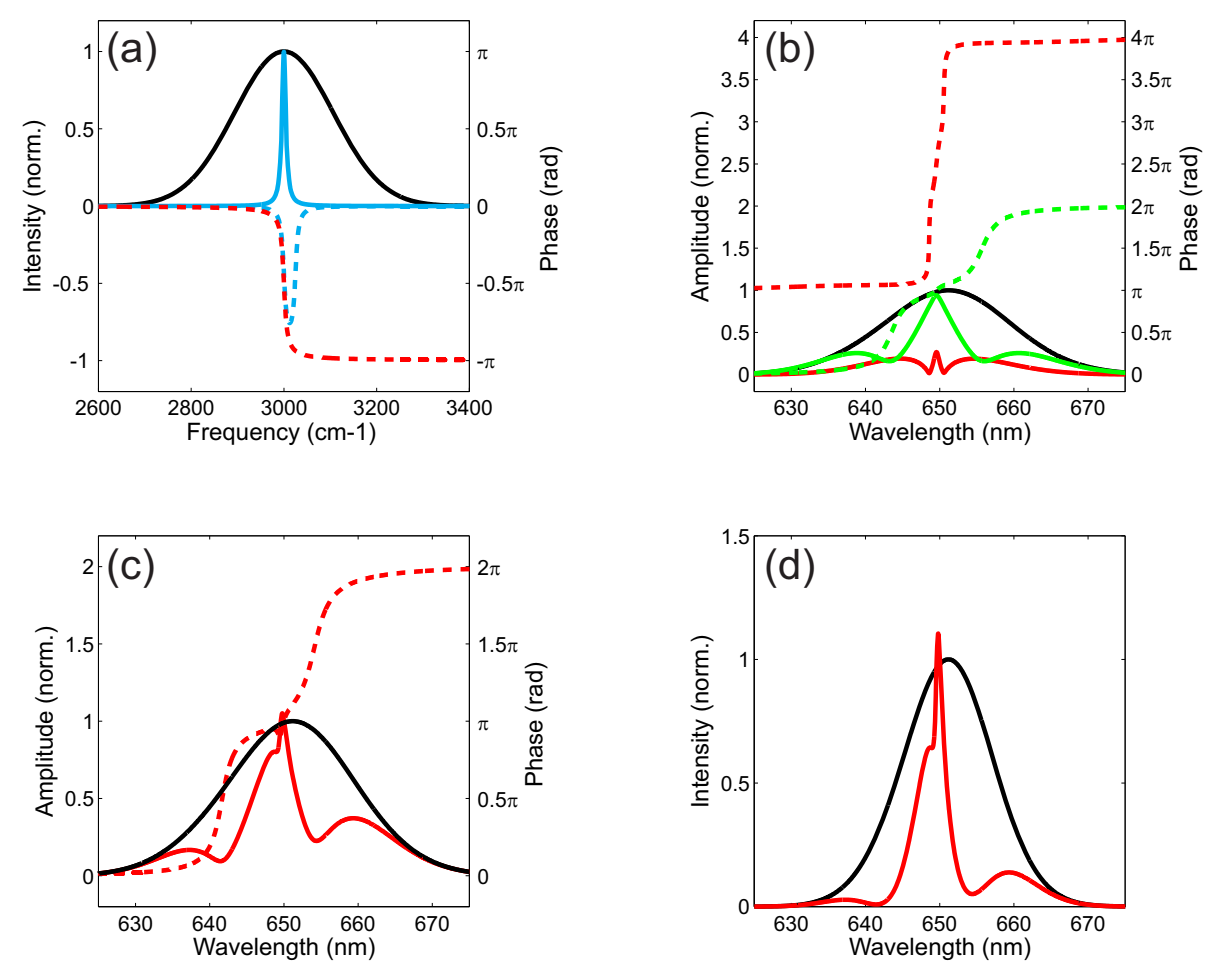

Figure 2.5: CARS response for a negative $\pi$ phase step. (a) $-\Im\left(\chi^{(3)}\right)$ (cyan solid) and phase (cyan dashed) of the vibrational resonance. Furthermore, the pump and probe pulse intensity (black) and phase (red), shifted by the Stokes frequency, are shown. (b) Resonant (red) and nonresonant (green) amplitude (solid) and phase (dashed) of the CARS signal for a pump and probe pulse with a negative $\pi$ phase step. The CARS amplitude for an unshaped pump and probe pulse (black) is shown for comparison. (c) Total CARS amplitude (red solid) and phase (red dashed) for a pump and probe pulse with a negative $\pi$ phase step. The CARS amplitude for an unshaped pump and probe pulse (black) is shown for comparison. (d) Total CARS intensity for a pump and probe pulse with a negative $\pi$ phase step (red) and for an unshaped pump and probe pulse (black). 

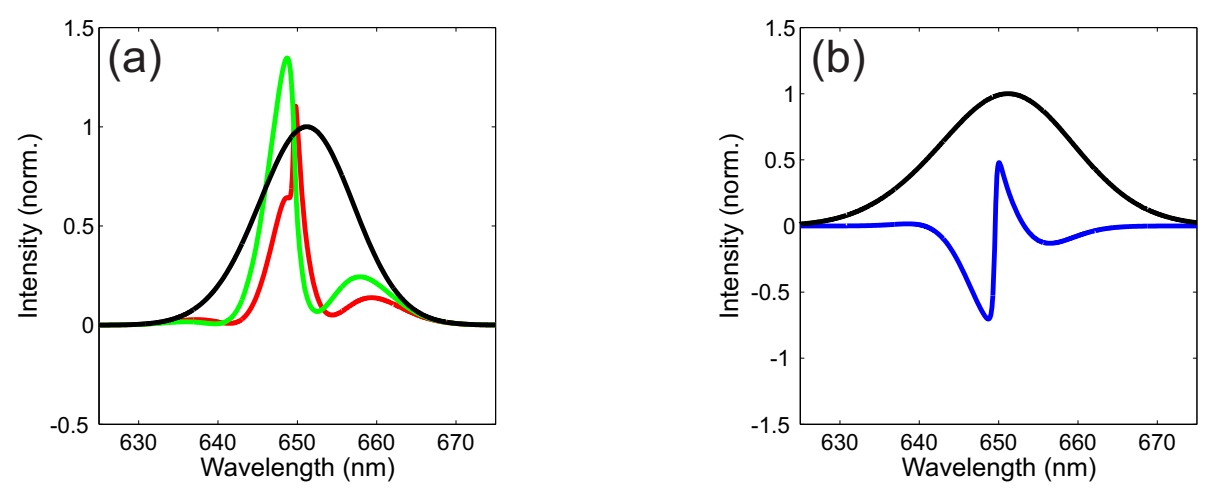

Figure 2.6: (a) CARS spectrum for a positive (green) and negative (red) $\pi$ phase step centered at the resonance. (b) Difference spectrum (blue) obtained by subtracting the positive step spectrum from the negative step spectrum. In $(a, b)$ the CARS spectrum for an unshaped pulse is shown for comparison (black).
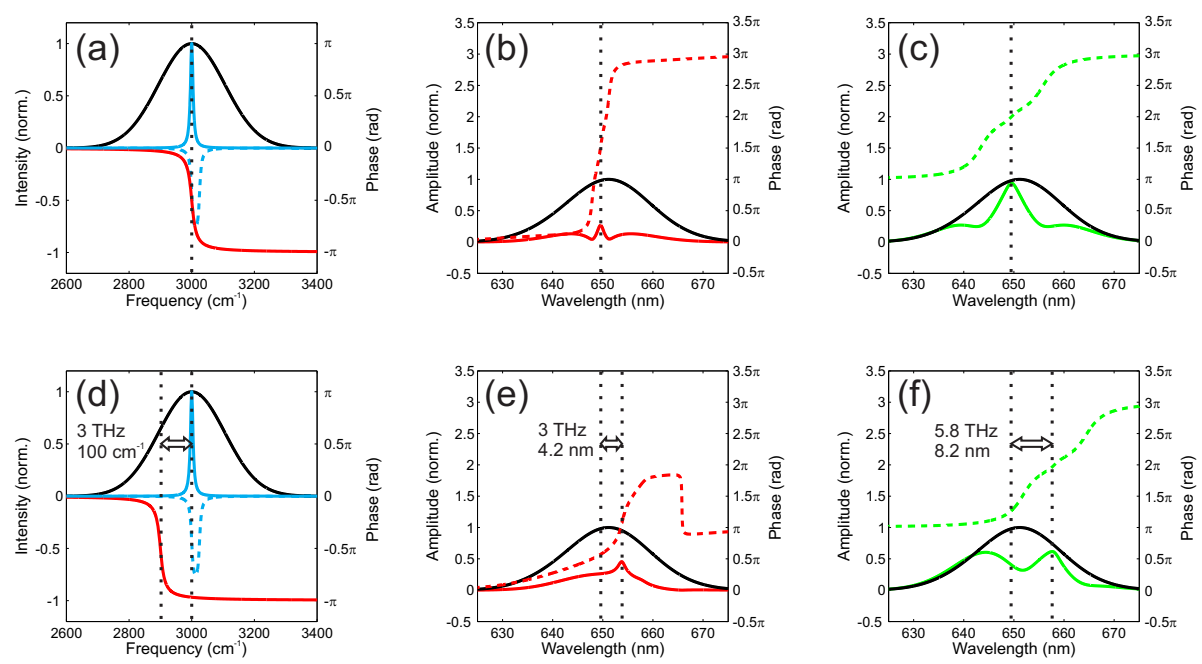

Figure 2.7: CARS fields for a negative $\pi$ phase step on resonance (a-c) and off resonance (d-f). (a,d) $-\Im\left(\chi^{(3)}\right)$ (cyan solid) and phase (cyan dashed) of the vibrational resonance. Furthermore, the pump and probe pulse intensity (black) and phase (red), shifted by the Stokes frequency, are shown. (b,e) Resonant amplitude (red solid) and phase (red dashed) of the CARS signal for a pump and probe pulse with a negative $\pi$ phase step. (c,f) Non-resonant amplitude (green solid) and phase (green dashed) of the CARS signal for a pump and probe pulse with a negative $\pi$ phase step. In $(b, c, e, f)$ the CARS amplitude for an unshaped pump and probe pulse (black) is shown for comparison. 
in the distance over which the peak in the non-resonant contribution is shifted is caused by the finite slope of the phase step. For an infinitely sharp phase step, the displacement of the peak in the non-resonant contribution is equal to twice the displacement of the phase step.

The peak in the resonant signal will be displaced by the same amount as the phase step, because the moving phase step in the probe pulse is convoluted with the stationary phase step induced by the vibrational resonance. For the resonant contribution, the pump pulse is multiplied by the vibrational response of the molecule before the convolution with the probe pulse, so if the phase step in the pump pulse is located offresonance, it will have no effect, since the amplitude of the molecular response is zero in that region. The resonances of a molecule have an intrinsic $\pi$ phase step in their response however, which is at a fixed location. The $\pi$ phase step in the probe is displaced, while the $\pi$ phase step of the molecular response is fixed. The crossing point of both phase steps in the convolution is thus displaced by the same amount as the applied phase step. For a scanning $\pi$ phase step this gives rise to the resonant feature that is displaced by the same amount as the phase step in the pump and probe pulse. The effect of a displaced phase step on the resonant CARS signal is shown in figure 2.7(b,e).

The shape and strength of the features caused by the phase step in the pump and probe spectrum changes as a function of the location of the phase step. These changes are also visible in the spectrally integrated CARS intensity. By scanning the phase step through the pump and probe spectrum, we obtain a graph of CARS intensity as a function of phase step location, as shown in figure 2.8(a).

It can be seen from figure 2.8(a) that the integrated CARS intensity as a function of $\pi$ phase step location yields information on the location of vibrational resonances. A sharp increase in CARS signal strength is observed at the location of the resonance. The difference in signal strength at both sides of the resonance for the negative phase step is caused by changes in interference caused by the phase shift associated with the molecular resonance.

As discussed earlier in this section (figure 2.6), we can remove the non-resonant background by subtracting the signals obtained for the positive and negative phase step from each other. This procedure can be done for the integrated intensity as a function of phase step location 

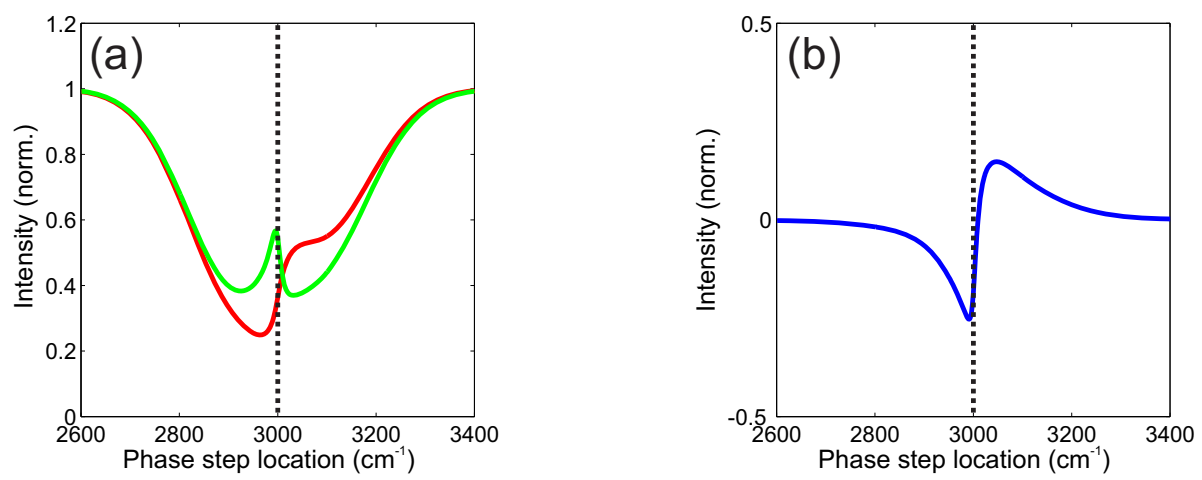

Figure 2.8: (a) Integrated CARS signal as a function of phase step location (shifted by the Stokes frequency) for a positive (green) and negative (red) $\pi$ phase step. (b) Integrated difference signal obtained by subtracting the intensities for the positive step scan from the negative step scan. The dashed black lines indicate the center frequency of the vibrational resonance.

as well, as shown in figure 2.8(b).

The removal of the non-resonant background contribution allows for detection of very weak resonances, which may not be apparent in the individual positive and negative step measurements. This effect is illustrated in figure 2.9, where the non-resonant background is increased to $200 \%$ (peak to baseline).

Figures 2.8(b) and 2.9(b) show that a resonance produces a visible jump in intensity in the integrated difference signal. Also, the broad dip in the individual graphs (figures 2.8(a) and 2.9(a)), caused by the interference in the non-resonant signal, is no longer visible, because the non-resonant contributions cancel.

In the case of multiple resonances, every resonance generates its own peak in the integrated signal. Strong resonances can hinder the detection of weaker resonances in the integrated signal, as illustrated in figure 2.10. In this case, three resonances are simulated, with center frequencies of $2980 \mathrm{~cm}^{-1}, 3000 \mathrm{~cm}^{-1}$, and $3050 \mathrm{~cm}^{-1}$, FWHM linewidths of 10 $\mathrm{cm}^{-1}, 10 \mathrm{~cm}^{-1}$, and $20 \mathrm{~cm}^{-1}$ and relative strengths of $0.3,1$, and 0.5 , respectively. 

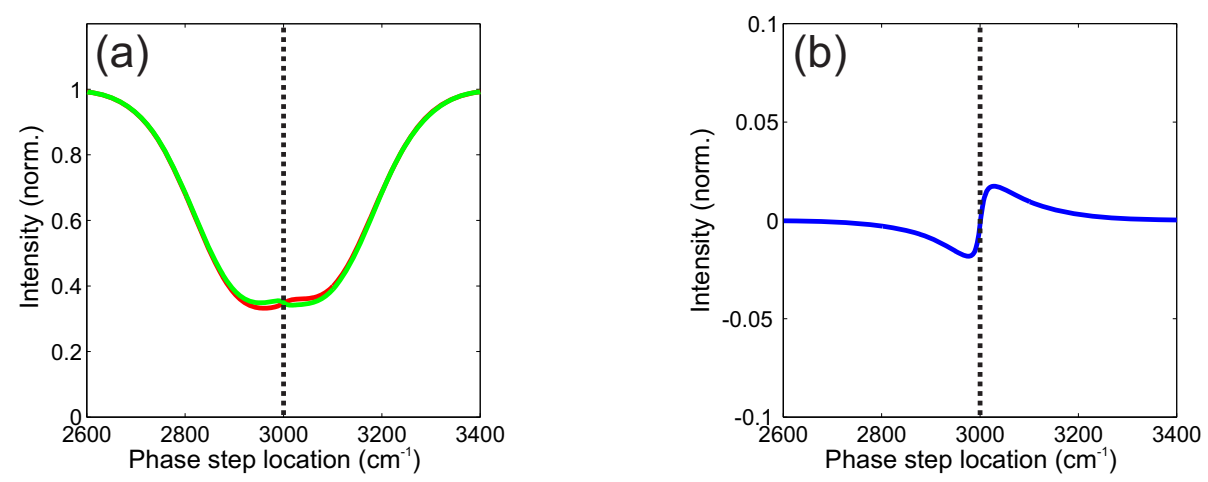

Figure 2.9: (a) Integrated CARS signal as a function of phase step location (shifted by the Stokes frequency) for a positive (green) and negative (red) $\pi$ phase step in the case of a weak resonance. (b) Integrated difference signal obtained by subtracting the intensities for the positive step scan from the negative step scan. The dashed black lines indicate the center frequency of the vibrational resonance.


Figure 2.10: (a) Integrated CARS signal as a function of phase step location (shifted by the Stokes frequency) for a positive (green) and negative (red) $\pi$ phase step in the case of three resonances. (b) Integrated difference signal obtained by subtracting the intensities for the positive step scan from the negative step scan. The dashed black lines indicate the center frequencies of the vibrational resonances. 


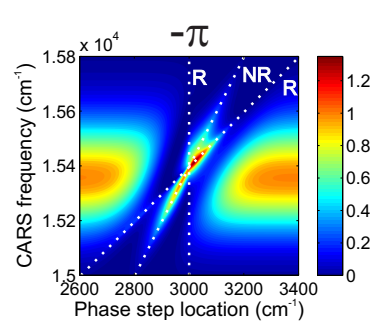

(a)

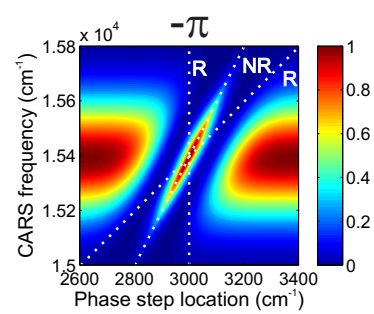

(d)

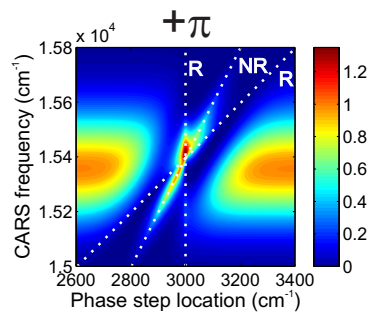

(b)



(e)

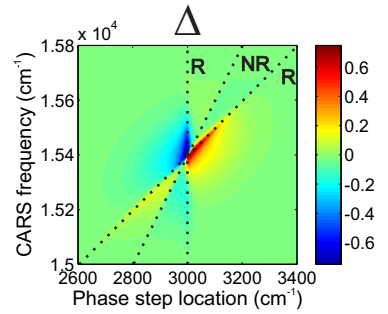

(c)

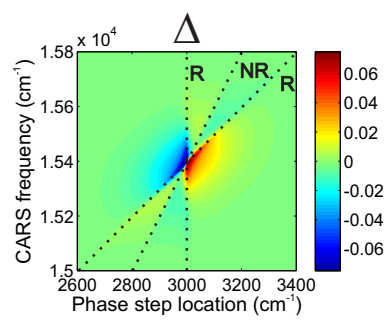

(f)

Figure 2.11: 2D spectra for a single strong (a-c) and weak (d-f) resonance. (a,d) $2 D$ spectrum for a negative $\pi$ phase step. (b,e) $2 D$ spectrum for a positive $\pi$ phase step. (c,f) $2 D$ difference spectrum obtained by subtracting the $2 D$ spectrum for the positive phase step from the $2 D$ spectrum for the negative phase step. The black and white dashed lines indicate the non-resonant contributions (NR, with slope 2) and the resonant contributions ( $R$, vertical and with slope 1$)$.

\subsubsection{2-Dimensional spectra}

Instead of looking at the integrated CARS signal as a function of phase step location, it is also possible to analyze the spectrally resolved CARS signal to find (weak) resonances more easily. This is done by plotting a 2-dimensional spectrum, with the CARS spectrum on the y-axis and the frequency of the location of the phase step, shifted by the Stokes frequency, on the x-axis. The intensity is plotted using a colormap. An example of such a 2D spectrum is presented in figure 2.11.

In figure 2.11(a,b) a negative and positive $\pi$ phase step scan are simulated for a single resonance with a center frequency of $3000 \mathrm{~cm}^{-1}$ and a FWHM linewidth of $20 \mathrm{~cm}^{-1}$. The resulting 2D spectra have nonresonant lobes at the edges and a broad non-resonant line with a slope of 2 in the center. In the $2 \mathrm{D}$ spectrum of the positive $\pi$ phase step scan 


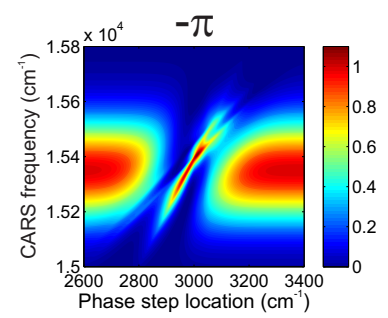

(a)

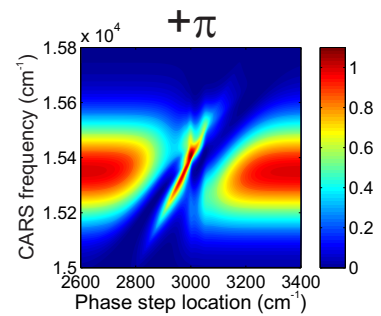

(b)

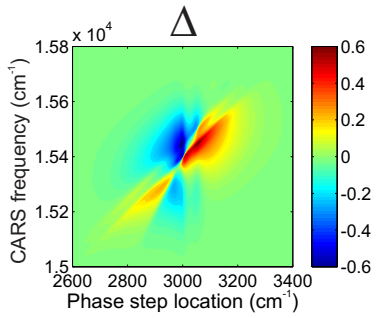

(c)

Figure 2.12: $2 D$ spectra in the case of three resonances. (a) $2 D$ spectrum for a negative $\pi$ phase step. (b) $2 D$ spectrum for a positive $\pi$ phase step. (c) 2D difference spectrum obtained by subtracting the $2 D$ spectrum for the positive phase step from the $2 D$ spectrum for the negative phase step.

there is a vertical distortion of the spectrum at the frequency of the vibrational resonance $(370 \mathrm{THz})$. Also a line with a slope of 1 is faintly visible. This line is due to the resonant contribution of the vibrational resonance and it crosses with the non-resonant line with a slope of 2 (taking the scaling of the axes into account) when the phase step in the pump and probe pulse overlaps with the vibrational resonance. The $2 \mathrm{D}$ spectrum for the negative phase step shows the same features, but in this case the sloped resonant line is more pronounced and the vertical distortion is less visible.

The resonance causes a vertical distortion and a line-shaped deformation with a slope of 1 in the $2 \mathrm{D}$ spectrum. The frequency of the resonance can be determined either by locating the position of the vertical distortion or by finding the frequency at which the resonant line (with a slope of 1 ) and the non-resonant center line (with a slope of 2) intersect.

The 2D spectra shown in figure 2.11(a,b) are still dominated by nonresonant background contributions. These non-resonant contributions can be removed by subtracting the $2 \mathrm{D}$ spectrum for the positive and negative phase step from each other. The resulting $2 \mathrm{D}$ difference spectrum is shown in figure 2.11(c).

In the case of multiple resonances, each resonance will have its own vertical and sloped line features (with a slope of 1 ), as can be seen in figure 2.12. Three resonances were simulated, with the same parameters as in section 2.5.2. 


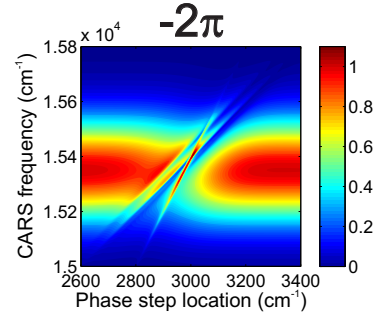

(a)

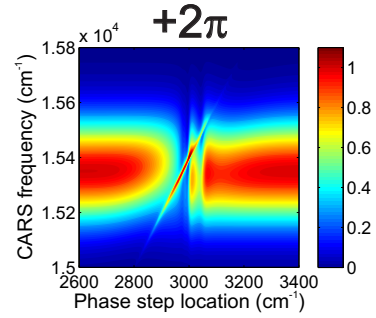

(b)

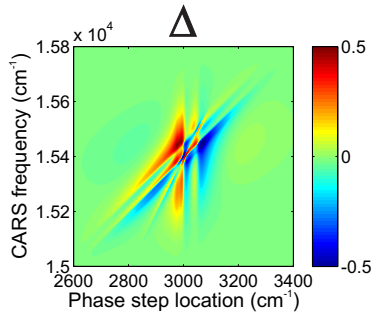

(c)

Figure 2.13: $2 D$ spectra in the case of three resonances, using a $2 \pi$ phase step. (a) 2D spectrum for a negative $2 \pi$ phase step. (b) $2 D$ spectrum for a positive $2 \pi$ phase step. (c) $2 D$ difference spectrum obtained by subtracting the $2 D$ spectrum for the positive phase step from the $2 D$ spectrum for the negative phase step.

\subsection{4 $2 \pi$ phase step}

Instead of using a $\pi$ phase step, it is also possible to use a $2 \pi$ phase step. Using the same techniques as for the $\pi$ phase step (described in section 2.5.2), resonances can be identified. However, the vertical and sloped lines caused by the resonant contributions to the CARS signal are much more pronounced. A $2 \mathrm{D}$ spectrum for a $2 \pi$ phase step scan on a molecule with three resonances is presented in figure 2.13. The resonances have the same parameters as in section 2.5.2.

For a $2 \pi$ phase step, there is a distinct difference compared to a $\pi$ phase step. Instead of having both vertical and sloped resonant features for both the negative step and the positive step, as is the case for a $\pi$ phase step, there are only vertical resonant features for the positive step and only sloped resonant features for the negative step. The $2 \pi$ phase step can be considered as a flat phase profile, except for a small region where it is sloped. The different wavelengths in the pump and probe pulse can therefore be considered to be in phase, except for the region where the phase step is located. This reduces the destructive interference in the generated CARS signal. 


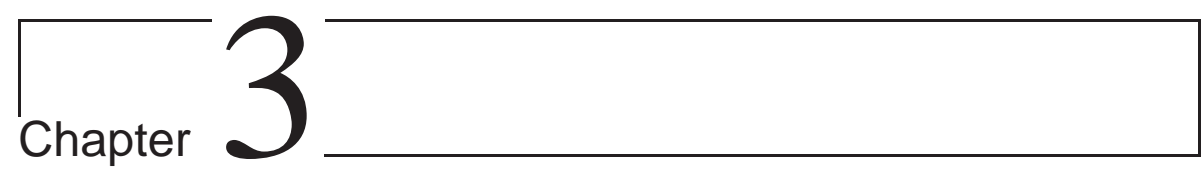

\section{Cross-polarized broadband SRS}

Stimulated Raman scattering (SRS) is a Raman-based process that has been gaining interest in recent years for use in spectroscopy and imaging. Most applications use a narrowband approach in which a single vibrational resonance is imaged at a time. In this chapter a broadband approach to SRS is described. Instead of using high-frequency optical modulators, as in most narrowband approaches, we apply a commonpath interferometer based on polarization rejection to detect the broadband SRS response. This chapter covers the experimental setup that was used, the obtained broadband SRS measurements, and suggestions on how to improve this technique. 


\subsection{Introduction}

Stimulated Raman scattering (SRS) is a Raman-based technique that relies on detection of stimulated loss in the pump beam or stimulated gain in the Stokes beam, as explained in section 2.3. Most SRS techniques to date work by applying a (high-frequency) modulation onto one of the input beams and detecting the resulting modulation on the other beam [20] or by spectral phase shaping [40]. Here, we present a different approach, which is based on common-path interferometry [53]. In this common-path interferometer, the two branches of the interferometer are spatially overlapped, which removes interferometric instabilities caused by for example air flow or temperature gradients.

This novel SRS technique does not require phase or amplitude modulation of the input beams and subsequently does not require lock-in amplified based detection. Our approach is broadband, providing spectrally resolved SRS data, without the need for a wavelength scannable excitation source.

\subsection{Setup}

\subsubsection{Working principle}

In our common-path interferometer the two different branches of the interferometer are formed by two orthogonal polarization states $\left(+45^{\circ}\right.$ and $-45^{\circ}$ linear polarization), which are time delayed with respect to each other. The two states are formed by letting the input beam propagate through a birefringent calcite crystal. When the incident polarization is at a $45^{\circ}$ angle with respect to the slow and fast axes of the crystal, $50 \%$ of the pulse is projected onto each polarization state (parallel to the fast and slow axes of the crystal). Furthermore, these pulses will be separated in time (as shown in figure 3.1), due to differences in group velocity for the two polarizations, where the amount of separation depends on the thickness of the birefringent crystal. A second calcite crystal, after the sample, is oriented such that the alignment of its fast and slow axes is exactly opposite to the first crystal. If the thickness of the second crystal matches the thickness of the first crystal, the two pulses generated in the first crystal are recombined into a single linearly polarized pulse. 


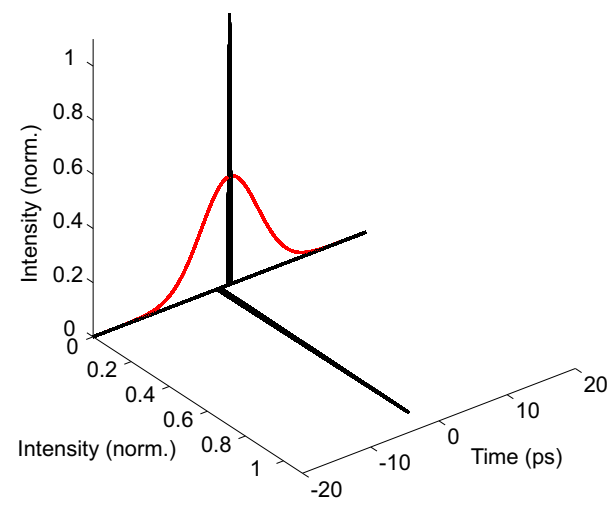

Figure 3.1: Generation of two orthogonally polarized pump pulses (black) by propagation through a calcite crystal. There is a 1.67 picosecond time delay between the two pulses. The 10 picosecond Stokes pulse, synchronized and polarization matched with one of the pump pulses, is shown in red for comparison.
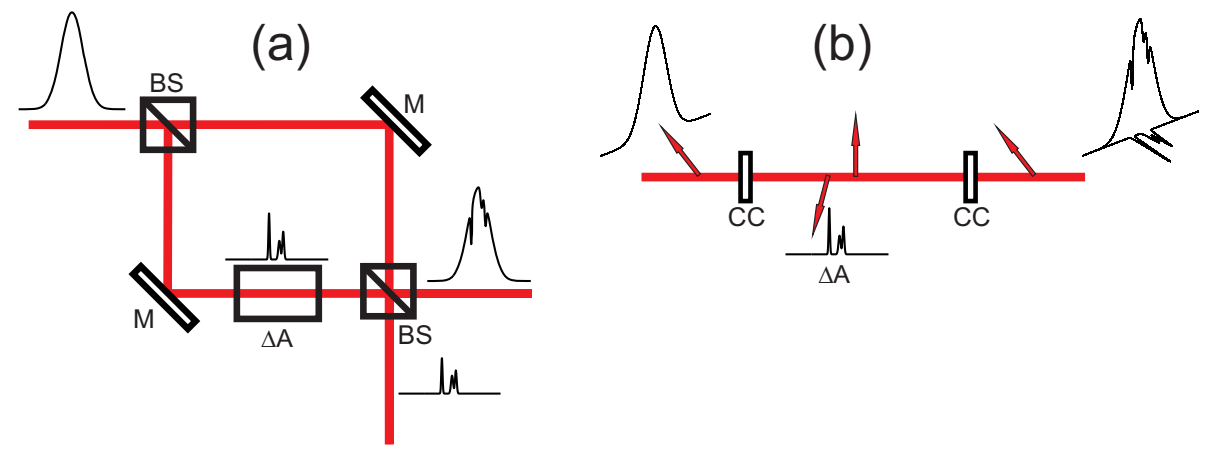

Figure 3.2: (a) Schematic drawing of a Mach-Zehnder interferometer. $B S=$ Beamsplitter, $M=$ Mirror, $\Delta A$ is an element that absorbs at certain wavelengths. (b) Schematic drawing of a polarization based common-path interferometer. $C C=$ Calcite crystal, $\Delta A$ is an element that absorbs at certain wavelengths and for only one of the polarizations in the interferometer. The arrows indicate polarization angles. 
The two orthogonal polarization states can be considered as two branches in a Mach-Zehnder interferometer, and the two calcite crystals can be considered as the beamsplitters in the Mach-Zehnder interferometer (figure 3.2(a)). If both branches in a Mach-Zehnder interferometer are identical, then the two branches will destructively interfere at one output of the recombining beamsplitter and constructively interfere at the other output, reconstructing the input state. If an absorption or optical path length difference is introduced in one of the branches, there will no longer be complete destructive interference and subsequently there will be light at both output ports.

In the case of our common-path interferometer, the same effect occurs with the different polarization states (figure 3.2(b)). If one of the polarizations in the interferometer is affected by an absorption, the two branches will no longer recombine to the exact input state at the exit crystal. The input polarization is defined by placing a polarizer in the pump beam. By placing an analyzer at $90^{\circ}$ degrees with respect to the input analyzer, the input polarization is rejected and the transmitted signal is the signal of the other output port of the interferometer, which contains only signal caused by the difference in absorption in the two branches.

In the SRS process, a pump photon is absorbed and a Stokes photon is emitted, leaving the molecule in a vibrational state. This means that there is an absorption in the pump beam, which can be detected using the common-path interferometer, provided that the absorption only occurs in one of the two polarization states. SRS is a polarization dependent process, and the SRS contribution is maximized when the pump and Stokes beams share the same polarization. The SRS signal is severely reduced for orthogonally polarized pump and Stokes beams [25]. We match the polarization of the Stokes beam with one of the polarization states of the pump beam in the interferometer (meaning it has a linear polarization that is offset by $45^{\circ}$ degrees with respect to the incoming pump beam). Because the two polarization states of the pump beam in the interferometer are orthogonal, SRS results for only one of the polarizations.

The SRS process occurs only for combinations of pump and Stokes photons that have an energy difference that corresponds to a vibrational resonance. Because the Stokes pulse is narrowband, it can only generate SRS with a single wavelength pump photon for each different 


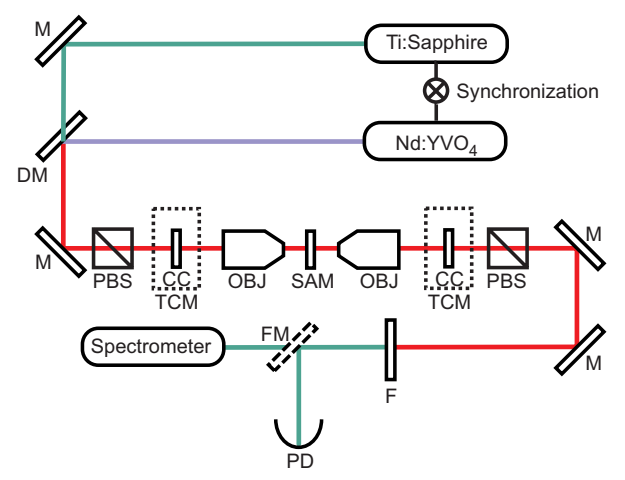

Figure 3.3: Schematic drawing of the broadband SRS setup. $M=$ Mirror, DM = Dichroic Mirror, PBS = Glan-Laser Polarizing Beamsplitter, $C C=$ Calcite Crystal, $T C M=$ Temperature Controlled Mount, OBJ = Microscope Objective, SAM = Sample, $F=$ Filter, FM = Flip Mirror, $P D=$ Photodiode.

vibrational resonance. These wavelengths will therefore experience an absorption due to the SRS process. This absorption will cause the light at this wavelength to be recombined into a slightly different polarization, which is not completely rejected by the analyzer. The different vibrational resonances can therefore be detected by looking at the spectrally resolved output signal after the analyzer.

\subsubsection{Overview}

The setup uses two oscillators. The first is a Ti:Sapphire oscillator (KMLabs) that acts as the pump beam, with a center wavelength around 800 $\mathrm{nm}$, a pulse length of 70 femtoseconds and an average output power of $400 \mathrm{~mW}$ at a $80 \mathrm{MHz}$ repetition rate. This oscillator is pumped by a continuous wave $532 \mathrm{~nm}$ laser (Spectra Physics Millennia VI). The second oscillator is a Nd: $\mathrm{YVO}_{4}$ oscillator (Spectra Physics Vanguard), which acts as the Stokes beam. This laser has a center wavelength of $1064.3 \mathrm{~nm}$ and a pulse length of 10 picoseconds at a $80 \mathrm{MHz}$ repetition rate and $900 \mathrm{~mW}$ average power. The repetition rate of both lasers is synchronized by a two-stage electronic and optical feedback system, which is described in section 3.2.3. A schematic representation of the common-path interferometer setup is shown in figure 3.3 .

The calcite crystals (Casix) are $20 \mathrm{~mm} \times 20 \mathrm{~mm}$ and $3 \mathrm{~mm}$ thick 
and are cut along the $\alpha$-axis. The crystals are coated with a $\mathrm{MgF}_{2}$ antireflection coating for 760-860 $\mathrm{nm}$. The tolerances on the dimensions of the crystals are specified to be $\pm 0.1 \mathrm{~mm}$. The calcite crystals are placed in home-built temperature controlled mounts to control the temperature within $0.01{ }^{\circ} \mathrm{C}$, which influences the applied optical retardance. The difference between the ordinary and extraordinary refractive index equates to a time delay between the two pump pulses of 1.67 picoseconds at room temperature.

The temperature controlled crystal mounts consist of aluminum encasings for the crystals, with two embedded temperature sensors. There are also eight resistors on the outside of the mount, four on each side, along the edges of the crystal, as shown in appendix B. These resistors heat the crystal mounts and the crystals inside them. The mounts are isolated from the optical table by a non heat conducting plastic layer, to minimize heat leakage into the optical table, and thereby reduce the current load on the resistors that is required to maintain a stable temperature. The temperature is stabilized using PID controllers (Omron E5EN-H) on the current supplies (Delta Elektronika ES030-5), and the signal from temperature sensors inside the mounts is used as feedback signal. A temperature stability of $\pm 0.01^{\circ} \mathrm{C}$ is obtained.

The Glan-Laser polarizers (Thorlabs GL10-B) are calcite-based airspaced polarizing beamsplitters, with a specified rejection ratio of $1: 10^{5}$. The excitation objective is a 0.65 numerical aperture (NA) infra-red objective (Nachet Pl.Fl.) and the collection objective is a $0.5 \mathrm{NA}$ extra long working distance objective (Nikon M-plan 40x). The spectrometer is a silicon based 3648 pixel spectrometer (Avantes AvaSpec-3648-USB2) with a resolution of $0.32 \mathrm{~nm} /$ pixel. An incident power of $100 \mathrm{~mW}$ for the pump beam and $600 \mathrm{~mW}$ for the Stokes beam was used.

Using a constant pump amplitude approximation, the expected stimulated Raman gain (and loss) can be calculated for a steady state system using equations 3.1 and 3.2 [23].

$$
\begin{array}{r}
m_{S}(z)=m_{S}(0) e^{g I_{p} z} \\
\frac{\Delta I_{p}}{I_{p}}=\frac{\Delta I_{S}}{I_{S}}=\frac{m_{S}(z)-m_{S}(0)}{m_{S}(0)}
\end{array}
$$

where $m_{S}(0)$ is the number of photons in the incident Stokes beam, $m_{S}(z)$ is the number of photons in the Stokes beam after an interaction length $z, g$ is the Raman gain, $I_{p}$ is the intensity of the pump beam, and 
$I_{s}$ is the intensity of the Stokes beam.

To estimate the stimulated Raman loss in our experiment we use a Raman gain of $0.1512 \mathrm{~cm} / \mathrm{GW}$. This value is based on the $1.17 \mathrm{~cm} / \mathrm{GW}$ Raman gain reported for the $2925 \mathrm{~cm}^{-1}$ resonance of acetone, using $530 \mathrm{~nm}$ excitation light [54]. The Raman gain is related linearly to the scattering cross section, which has a 4th power dependence on the frequency difference between the excited vibrational resonance and the pump light [55]. Therefore we scale the Raman gain by a factor of $(12500-2925)^{4} /(18868-2925)^{4}$ to compensate for the change in pump wavelength from $530 \mathrm{~nm}\left(18868 \mathrm{~cm}^{-1}\right)$ to $800 \mathrm{~nm}\left(12500 \mathrm{~cm}^{-1}\right)$. An interaction length of $1.9 \mu \mathrm{m}$ is chosen, which equals the length of the axial point spread function for a 0.65 NA objective, and a pump power of 100 $\mathrm{mW}$ is used. Using these parameters, the stimulated Raman loss is calculated to be $3^{*} 10^{-8}$. For our broadband pulsed system the stimulated Raman loss will most likely be larger due to the increased efficiency of the SRS process if both the pump and Stokes are pulsed (see equation 2.10). Stimulated Raman loss values of $<10^{-4}$ have been reported for a pulsed picosecond system by Xie et al. [20].

\subsubsection{Laser synchronization}

\section{Electronic synchronization}

To overlap the pulses in time in the sample, it is necessary to synchronize the repetition rate of both lasers. Our approach is based on a two-stage feedback system that actively synchronizes the repetition rate of the Ti:Sapphire oscillator to that of the $\mathrm{Nd}: \mathrm{YVO}_{4}$ oscillator. The first stage is an electronic feedback system, followed by the second stage optical feedback system.

In the electronic feedback stage, the pulse trains of both lasers are measured using ultrafast photodiodes. The Ti:Sapphire pulses are measured using a silicon photodiode and for the $\mathrm{Nd}: \mathrm{YVO}_{4}$ oscillator an InGaAs photodiode is used, due to the better sensitivity of InGaAs at $1064.3 \mathrm{~nm}$ when compared to silicon. The signals from both photodiodes are filtered by a low-pass filter with a $91 \mathrm{MHz}$ cut-off frequency, amplified, and filtered again with a $91 \mathrm{MHz}$ cut-off low-pass filter. The amplified and filtered signals from both photodiodes are then combined in a mixer, which extracts the difference between the two signals. The difference is regulated to zero to avoid sensitivity to amplitude fluctu- 


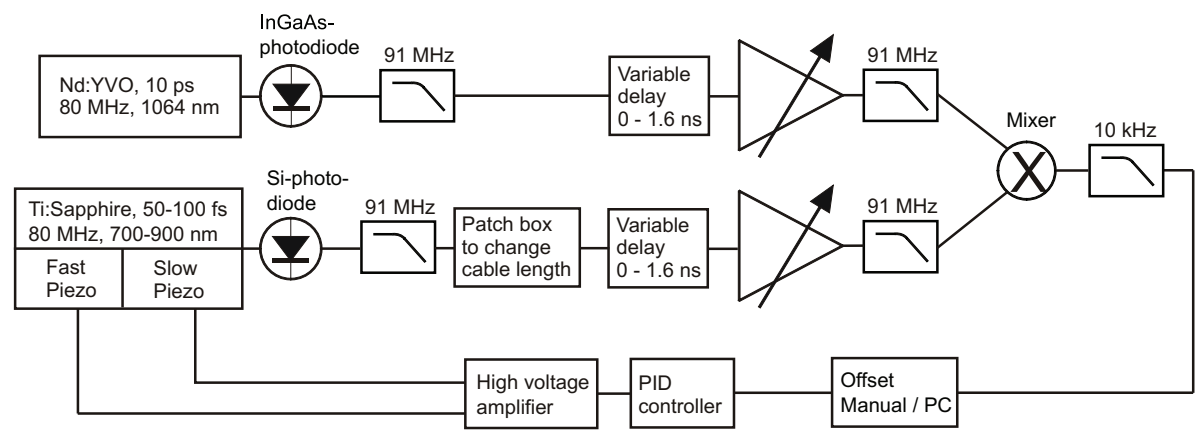

Figure 3.4: Schematic of the electronic feedback system. Image adapted from [56].

ations. A schematic of the electronic feedback system is shown in figure 3.4 .

First, the difference frequency is manually reduced to less than $20 \mathrm{~Hz}$ by changing the Ti:Sapphire cavity length with a motorized translation stage underneath the output coupler. Subsequently, the difference signal is used in a feedback loop that controls two piezo actuators. One of the piezo actuators is mounted on the output coupler of the Ti:Sapphire oscillator and is used for low frequency adjustments $(<3 \mathrm{~Hz})$, with a travel range of $30 \mu \mathrm{m}$. The other piezo actuator is mounted on the high reflector of the Ti:Sapphire oscillator and is used for fast adjustments $(>3 \mathrm{~Hz}$ ), with a travel range of $15 \mu \mathrm{m}$. The fast piezo actuator is driven by the high frequency part of the feedback signal and the slow piezo actuator is driven by the low frequency part of the feedback signal. With the repetition rates synchronized, there is a constant time delay between the two pulse trains. This delay can be adjusted by a patch cable box in the Ti:Sapphire signal branch, providing a tunable delay of up to 127.5 ns with a 0.5 ns stepsize. Fine adjustments can be made by adjustment of a tunable low-pass filter in either signal branch. By tuning the cut-off frequency of the low-pass filter, the phase shift that it applies to the transmitted signal will change, providing very precise control over the applied time delay.

With the electronic feedback, there is residual timing jitter between the pulses, in the order of a couple of picoseconds, and low frequency drift caused by changes in the pathlength through the coaxial cables [56]. Because the laser pulses are $70 \mathrm{fs}$ and $10 \mathrm{ps}$ in duration, this timing jitter will have a substantial effect on the temporal overlap between 
both pulses. Therefore, a second stage stage optical feedback circuit is used.

\section{Optical synchronization}

The optical synchronization is used as a more sensitive feedback mechanism once electronic locking has been obtained. A part of the Ti:Sapphire laser beam is split off using a pellicle beamsplitter and a part of the $\mathrm{Nd}: \mathrm{YVO}_{4}$ beam is split off using a half-wave plate and a polarizing beamsplitter cube. These beams are combined, overlapped on a dichroic mirror and focused into a BBO crystal, which is set at the optimal angle for sum-frequency generation between the two beams. The delay between the oscillators is adjusted such that the pulses overlap in the BBO crystal, causing sum-frequency light to be generated.

The sum-frequency light is detected on a silicon photodiode. The excitation light, as well as the second harmonic light that is generated from both input wavelengths, is rejected by a set of filters in front of the photodiode (Thorlabs, FEL0450 and FES0500). The photodiode signal is amplified using a transimpedance amplifier $\left(10^{8} \mathrm{~V} / \mathrm{A}, \pm 10 \mathrm{kHz}\right.$ bandwidth) and a controllable DC voltage offset is subtracted from this amplified signal. This DC offset has an amplitude equal to the average between the signal for maximum sum-frequency generation and the signal in case of no sum-frequency generation (zero). The result is a $0 \mathrm{~V}$ signal at the point where the Ti:Sa pulse is on the wing of the Nd:YVO pulse (see figure 3.5). Depending on the direction of timing drift, this will cause the amount of overlap between the pulses to either increase or decrease, which in turn causes the amount of sum-frequency that is generated to either increase or decrease. This sum-frequency signal is used in a feedback loop that provides additional timing stability between the pulses compared to the electronic feedback loop.

Using the optical synchronization, the timing jitter between the two oscillators is reduced. A reduction of the residual timing jitter to $60 \mathrm{fs}$ rms in a bandwidth of 0.04 to $100 \mathrm{~Hz}$ has been achieved [56].

\subsection{Results and discussion}

Broadband SRS measurements were performed on pure acetone $(\geq 99 \%$, Sigma-Aldrich). The acetone was placed in a glass cuvette with an inter- 


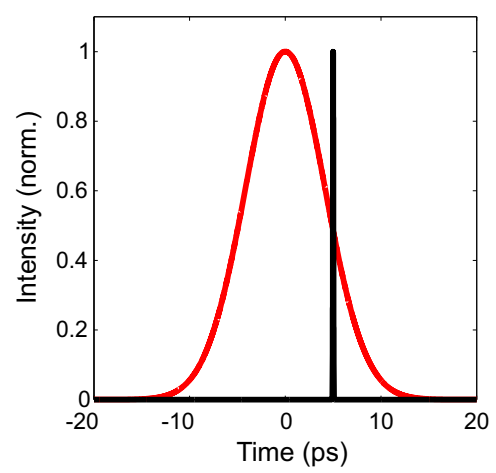

Figure 3.5: Pulse offset in the optical synchronization, where the Ti:Sa pulse (black) is on the wing of the $\mathrm{Nd:YVO}$ pulse (red).

action length of $1 \mathrm{~mm}$. The spectrum of the pump light that is transmitted through the second Glan-Laser polarizer (analyzer) was measured with and without the Stokes beam present in the sample. The results are shown in figure 3.6(b). Figure 3.6(a) shows the spectrum of the pump pulse from the Ti:Sa oscillator, as well as the spontaneous Raman spectrum from acetone, offset by the frequency of the Stokes pulse.

It can be seen from figure 3.6(b) that pump light is transmitted through the analyzer even when the Stokes beam is not present in the sample (black dashed line). The absence of the Stokes beam prevents stimulated Raman processes from occuring. The pump light that is still coming through the analyzer can be attributed to a number of causes.

First, the pump beam will be slightly depolarized by the excitation and collection objective in the interferometer. Without the objectives, the rejection ratio of the pump light in the interferometer was measured to be 355:1. With the objectives, the rejection ratio decreases to 130:1.

Another contribution is due to the imperfect recombination of polarization states over the complete spectrum. This imperfect recombination is caused by a difference in thickness between the two calcite crystals.

Because the calcite crystals are birefringent, they have different refractive indices for the ordinary axis $\left(n_{o}\right)$ and extraordinary axis $\left(n_{e}\right)$. Since the optical path length is equal to the physical path length times the refractive index, there is a difference in optical path length between the different polarizations (travelling along the two different axes) that 

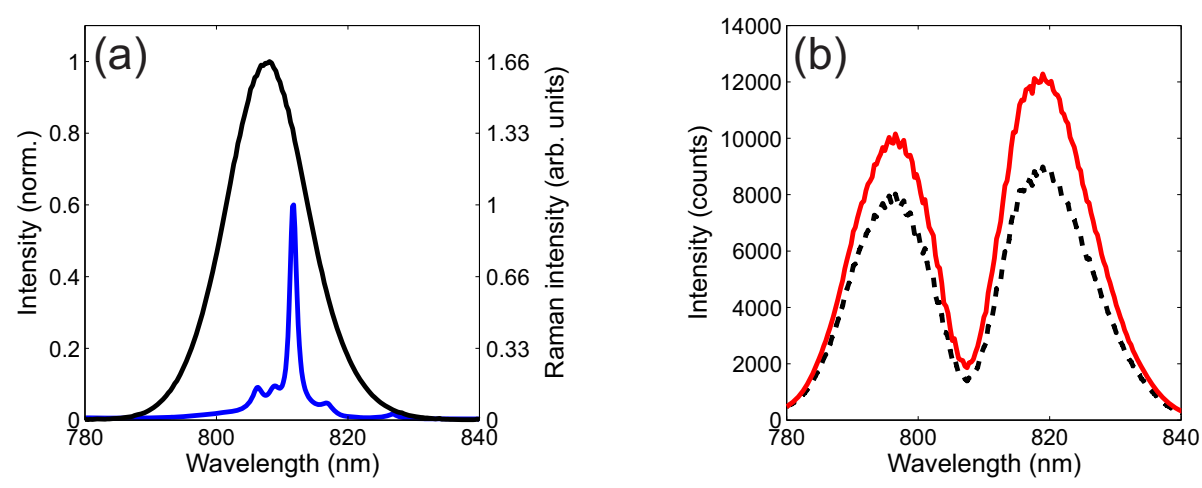

Figure 3.6: Broadband SRS measurement on acetone. (a) Normalized spectrum of the pump pulse (black) and the spontaneous Raman spectrum of acetone (blue), shifted by the Stokes frequency. (b) Transmission of the pump beam through the analyzer with (red, solid) and without (black, dashed) the Stokes beam present.

is linearly dependent on the length of the crystal. This difference in optical path length causes a time offset between the two pulses in the interferometer $\left(\Delta \tau=\left|n_{e}-n_{o}\right| L / c\right)$. If there is a difference in thickness between the two crystals, the second crystal will no longer compensate precisely the path length difference that is introduced by the first crystal, causing the polarization state of the recombined pulse to be misaligned with respect to the analyzer.

The extraordinary refractive index $\left(n_{e}\right)$ also varies with temperature [57], allowing one to compensate the mismatch in optical path length difference, induced by the difference in thickness, by applying a temperature difference between the crystals. Unfortunately, this dependence of the extraordinary refractive index with respect to temperature $\left(d n_{e} / d T\right)$ varies as a function of wavelength (see figure 3.7(a)). By changing the temperature, one therefore also changes the shape of the dispersion curve $d n_{e} / d \lambda$. Due to this change in the dispersion curve, tuning the temperature only compensates the path length mismatch for a single wavelength, as shown in figure 3.7(b). It can be seen that the residual path length difference increases for wavelengths that are further away from the wavelength that is being fully compensated. This effect is responsible for the dip and two side lobes in the transmitted spectrum.

It can be seen from figure 3.6(b) that there is an increase in tranmitted pump light when the Stokes beam is present in the sample. This 

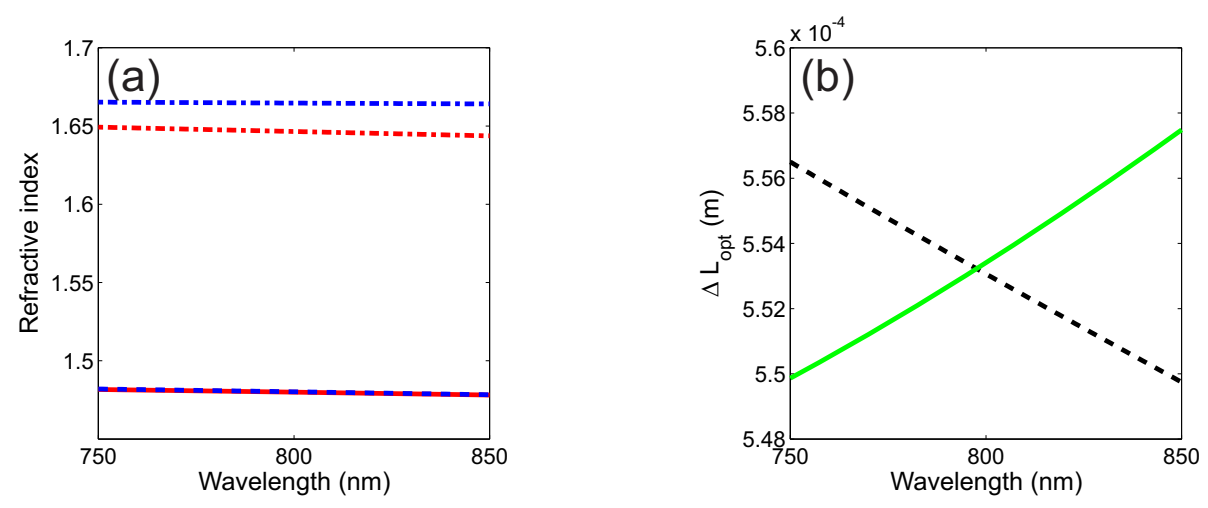

Figure 3.7: (a) Calculated refractive indices for calcite at two temperatures as a function of wavelength, using data from [57]. The extraordinary refractive indices are indicated by the dash-dot lines and the ordinary refractive indices are indicated by the dashed lines. The red lines represent calcite at $23{ }^{\circ} \mathrm{C}$ and the blue lines represent calcite at $60.7^{\circ} \mathrm{C}$. (b) Effect of temperature tuning to compensate a thickness difference between two calcite crystals. The black dashed line indicates the optical path length difference between the two crystal axes through a 3 $m m$ crystal at $23{ }^{\circ} \mathrm{C}$ and the the solid green line indicates the optical path length difference between the two crystal axes through a $3.32 \mathrm{~mm}$ crystal at $60.7^{\circ} \mathrm{C}$. 
increase extends over the full spectrum. However, SRS can only occur when the energy difference between a pump photon and a Stokes photon coincides with the energy of a vibrational mode in the sample. Since the bandwidth of the pump pulse is much larger than the the bandwidth necessary to cover all $\mathrm{C}-\mathrm{H}$ resonances in acetone (as shown in figure 3.6(a)), SRS can not be accountable for absorbing pump photons over the entire spectrum of the pump pulse.

There are other nonlinear processes that occur alongside the SRS process. Because this absorption of pump photons is detected only when both the pump and the Stokes beam are present in the sample, the process that is responsible for the absorption must be a nonlinear process that involves at least one pump photon and one Stokes photon. Acetone does not exhibit any second-order nonlinear responses, so this means the nonlinear process that causes this effect must be of at least third order. We attribute the absorption of pump photons over the full bandwidth of the pump pulse to non-resonant coherent anti-Stokes Raman scattering (CARS). Because CARS is a parametric process, it contains a non-resonant component, which is generated using the full bandwidth of the pump spectrum. This non-resonant CARS therefore shows up as a loss of pump photons over the full bandwidth of the spectrum.

Even though there is an absorption of pump photons over the entire spectrum, the absorption of pump photons due to SRS is localized to wavelengths that match with a vibrational resonance. To better visualize the absorption of pump photons, we look at the difference in intensity of the pump light transmitted by the analyzer with the Stokes beam present and with the Stokes beam not present in the sample and divide this by the spectrum of the case where there is no Stokes beam in the sample (equation 3.3). The result is shown in figure 3.8(b). The spectra obtained with and without the Stokes beam present, from which the normalized spectrum is obtained, are shown in figure 3.8(a).

$$
I_{\text {norm }}(\omega)=\frac{I_{S R S}(\omega)-I_{\text {ref }}(\omega)}{I_{\text {ref }}(\omega)}
$$

It can be seen from figure 3.8(b) that there is a small peak around $812 \mathrm{~nm}$, which corresponds to a Raman shift of $2918 \mathrm{~cm}^{-1}$. This peak is caused by the absorption of pump photons due to SRS at the very 

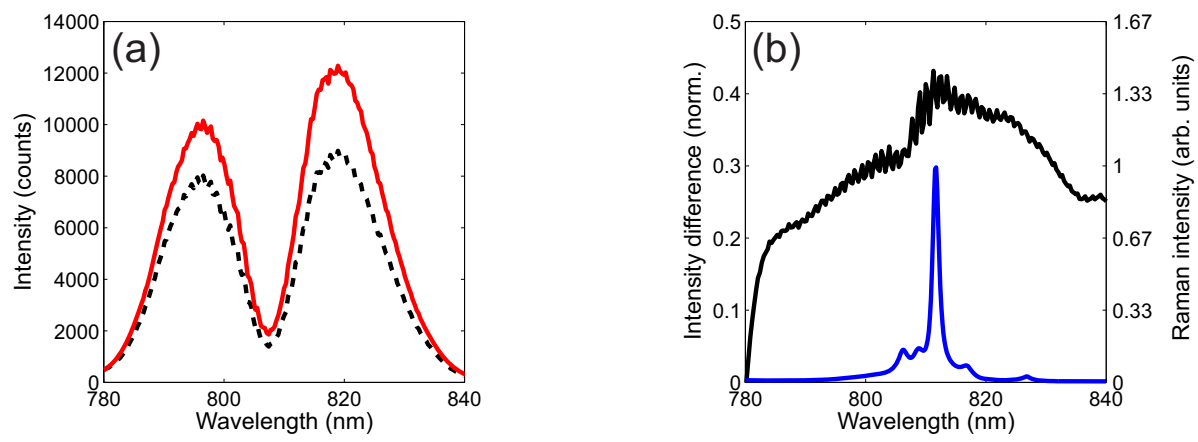

Figure 3.8: Broadband SRS measurement on acetone. (a) Transmission of the pump beam through the analyzer with (red, solid) and without (black, dashed) the Stokes beam present. (b) The normalized difference in transmitted pump light through the analyzer with and without the Stokes beam present. The normalized difference is obtained using equation 3.3. The spontaneous Raman scattering spectrum of acetone (blue) is shown for comparison.

strong C-H resonance of acetone located at $2923.8 \mathrm{~cm}^{-1}$ [58], showing the feasability to detect vibrational resonances using our common-path interferometer broadband SRS approach.

Unfortunately, the detection of vibrational resonances is limited by the depolarization due to the optics in the interferometer and the depletion of pump photons due to non-resonant CARS. The depolarization by the optics can be reduced by choosing lower NA microscope objectives at the cost of a reduction in signal.

To reduce the loss of pump photons by non-resonant CARS, one can use a narrowband pump pulse in combination with a broadband Stokes pulse. The non-resonant CARS contribution is governed by the frequency relation shown in equation 3.4.

$$
\omega_{C A R S}=\omega_{\text {pump }}-\omega_{\text {Stokes }}+\omega_{\text {pump }}
$$

The intensity dependence of this non-resonant CARS contribution is quadratic with respect to pump intensity and linear with respect to Stokes intensity (equation 3.5). 


$$
I_{C A R S} \propto I_{\text {pump }}^{2} I_{\text {Stokes }}
$$

While the SRS intensity is linearly dependent with respect to both the pump intensity and the Stokes intensity (equation 3.6).

$$
I_{S R S} \propto I_{\text {pump }} I_{\text {Stokes }}
$$

In our setup, the broadband pump pulse has a very short duration ( \pm $70 \mathrm{fs}$ ) compared to the Stokes pulse ( $\pm 10 \mathrm{ps}$ ), while the average power of both beams is of a similar order of magnitude. This means that the peak power of the pump pulse is much higher than that of the Stokes pulse. The CARS process scales quadratically with pump (peak) power, while the SRS process scales linearly with the pump (peak) power. Both the SRS process and the CARS process scale linearly with respect to the (peak) power of the Stokes pulse however. Hence it can be concluded that it is more advantageous to have a narrowband (low peak power) pump pulse and a broadband (high peak power) Stokes pulse instead.

For every CARS photon that is generated, there is an absorption of two pump photons as well as the generation of a Stokes photon. Therefore, the loss caused by non-resonant CARS in the pump beam is twice as large as the gain in the Stokes beam. It is therefore advantageous to measure the SRS on the Stokes beam, using a broadband Stokes beam and a narrowband pump beam.

It can be seen that there is a double advantage to using a narrowband pump pulse and a broadband Stokes pulse. It is expected that by using this combination of pulses, the detection sensitivity of this broadband SRS approach will be significantly enhanced. 
Chapter 3 


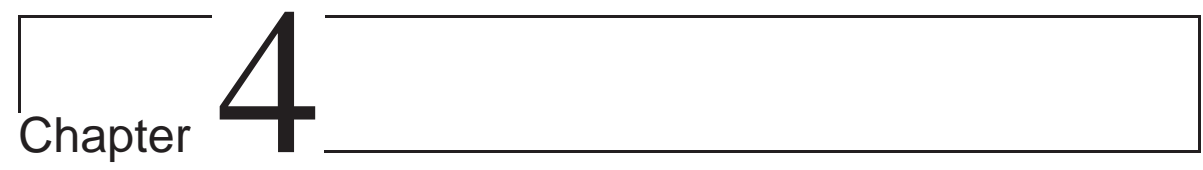

\section{Numerical optimization of CARS}

$C A R S$, a coherent nonlinear process, depends on the spectral phase of the applied light fields. Thus, precise control of the spectral phase enables us to influence the CARS process. In this chapter, an a-priori optimization approach for the spectral phase is described. The spectral phase can be optimized for different goals, for example obtaining maximum CARS signal from a single compound, or obtaining a better contrast for a single compound in a mixture of resonant compounds. To accurately model the CARS signal, the complex vibrational response of a molecule is required, which is extracted from spontaneous Raman scattering spectra. The model of the complex vibrational response is used to calculate and optimize the CARS signal for a given spectral phase of the input pulses. The CARS signal in our system is described by a convolution of the broadband pulse, multiplied by the vibrational response, and the broadband degenerate probe pulse. Direct calculation of the optimal excitation phase involves a deconvolution, which is not unambiguous. Therefore, we apply a numerical optimization to find the optimal excitation phase. At the basis of this optimization is a powerful evolutionary algorithm known as covariance matrix adaptation evolution strategy. 


\subsection{Introduction}

The CARS process can be influenced by controlling the spectral phase of one or more of the incident light fields. The challenge is to find the spectral phase profile that has the desired effect on the CARS signal generated by a certain (set of) molecule(s). Some of the more useful goals are maximizing the signal from a certain compound, enhancing the contrast of a certain compound with respect to other compounds present in a sample, or suppressing background signals. A number of approaches can be taken in order to find the optimal spectral phase that accomplishes the chosen goal.

Perhaps the most obvious approach is to calculate analytically the optimal phase function. Unfortunately, calculating the CARS response involves complex valued convolutions. Therefore an analytical solution requires a deconvolution, which is not unambiguous. One can construct phase profiles based on an intuitive understanding of the CARS response, considering isolated resonances. Examples of such phase shapes include sinusoidal phase [31], which generates a pulse train, linear chirps [59] or $\pi$ phase steps (see section 2.5).

It is also possible to use a fully experimental approach, for example to measure the CARS signal and use it as a feedback parameter in a closed-loop learning scheme. Such an approach is not guaranteed to find the global optimum, since the solution landscape can be quite complex and is especially ill-behaved when starting at low fitness values (far away from the optimum) [60] (see also section 4.6).

Our method combines the main ideas behind both of these approaches. We use a learning loop to calculate the CARS response of a molecule for a given set of input fields. This learning loop approach allows us to numerically pre-optimize the phase shape of our excitation pulse. The numerically optimized phase may not exactly match the experimental optimal pulse in the lab but should nonetheless be close to the global optimum. The solution landscape is ill-behaved far from the optimum, but it is smooth close to the optimum [60]. A second stage optimization in the lab is likely to be more succesful in finding the global optimum when starting out closer to its vicinity. In this chapter we describe our method for constructing a model of the complex vibrational response of a molecule based on spontaneous Raman scattering data and our method of numerical pre-optimization of the spectral phase of our excitation pulses 
for broadband CARS.

\subsection{The complex vibrational response}

\subsubsection{Modelling the vibrational response}

To model the CARS response of a molecule, the complex vibrational response, $\chi^{(3)}(\omega)$, is required. The complex vibrational response can be measured using heterodyne CARS [30], in which case a non-resonant reference signal is required to calibrate the measured phase. A more direct method of measuring the complex vibrational response is vibrational phase contrast CARS (VPC-CARS) [61], in which the reference signal is created using two competing pathways that interfere on the vibrational level. Other methods for retrieving the vibrational phase have been reported as well. These methods extract the complex vibrational response from a measured (broadband) CARS spectrum [45, 46, 47, 62, 63].

In our case, we use spontaneous Raman scattering spectra to determine the complex vibrational response. At first glance, one might think that spontaneous Raman scattering only yields information about the amplitude and frequency of vibrational resonances of a molecule and does not contain any phase information. However, the spontaneous Raman signal is proportional to the imaginary part of the complex response (see equation 4.1), and therefore contains both amplitude and phase information [64]. The vibrational response $\left(\chi^{(3)}\right)$ is described by a fourth rank tensor. Since the off-diagonal elements are very small and the depolarization ratio is low, we consider only contributions from the $\chi_{1111}^{(3)}$ tensor element in this work.

$$
I_{\text {Raman }} \propto \operatorname{Im}\left(\chi^{(3)}\right)=A\left(\chi^{(3)}\right) \sin \left(\phi\left(\chi^{(3)}\right)\right)
$$

The complex vibrational response of a molecule is a sum of different vibrational resonances. Since we are considering sample materials that are either in the solid phase or the liquid phase at room temperature, we do not have to be concerned about the different rotational levels in each vibrational resonance, since these rotational states will be homogeneously broadened by a large factor and are not individually resolvable at room temperature. As explained in chapter 2.1 we can consider each vibrational resonance as a damped driven harmonic 
oscillator. The complete vibrational response therefore becomes a sum of damped driven harmonic oscillator functions, with different oscillator strengths, damping factors and resonance frequencies, combined with a non-resonant term, as shown in equation 4.2 [23].

$$
\chi^{(3)}(\omega)=\chi_{N R}^{(3)}+\chi_{R}^{(3)}=\chi_{N R}^{(3)}+\sum \frac{A_{R}}{\omega_{R}^{2}-\omega^{2}-2 i \omega \gamma_{R}}
$$

One can not easily fit the parameters $\left(A_{R}, \omega_{R}, \gamma_{R}\right)$ of equation 4.2 , because they are not independent of each other. Often, resonances are overlapping and changing one or more parameters of a single resonance will modify an extended section of the vibrational response.

A second challenge is the number of unknown parameters that need to be fitted. Every resonance is described by three parameters and in order to accurately model the vibrational response over a region of several hundred wavenumbers, often up to 20 or more resonances are required. Therefore the parameter space that needs to be searched has a very high number of dimensions. In order to deal with these problem we employ an evolutionary algorithm to find the resonance parameters. The specific type of evolutionary algorithm that we employ is covariance matrix adaptation evolution strategy (CMA-ES) [65].

\subsubsection{Covariance matrix adaptation evolution strategy}

Covariance matrix adaptation evolution strategy (CMA-ES) is an optimization strategy that performs a stochastic search, while updating the covariance matrix of the optimization parameters. The covariance matrix contains both the necessary information for a principle component analysis, as well as the information on all the pairwise dependencies between variables. From the covariance matrix, the gradient in all directions of the search space is determined, which is used to adapt the direction of the search. CMA-ES also employs step-size control, which increases or decreases the step size based on the evolution of the searchpath, leading to a fast convergence and preventing the algorithm from getting stuck in a local optimum early on in the optimization. CMA-ES is especially useful in the case of non-separability and high dimensionality, where a deterministic approach is not feasible, and is robust with respect to ill-conditioned or noisy functions [66]. 


\subsubsection{Optimization of the vibrational response model}

We consider a sum of harmonic oscillators (equation 4.2) and fit the imaginary part of this function to the spontaneous Raman spectrum using CMA-ES. We define the fitness of our solution (which is a measure for the success of a solution) as the negative of the mean squared error between the imaginary part of our solution and the normalized spontaneous Raman intensity (equation 4.3).

$$
F(X)=-\sum_{n=\omega_{\min }}^{\omega_{\max }} \frac{\left(\frac{I_{\operatorname{Raman}}(n)}{I_{\operatorname{Raman}, \max }}-\operatorname{Im}(X(n))^{2}\right.}{N}
$$

where $\mathrm{F}(\mathrm{X})$ is the fitness of the complex solution function $\mathrm{X}$ that is being tested and $\mathrm{N}$ is the total number of points between $\omega_{\min }$ and $\omega_{\max }$ over which the spontaneous Raman spectrum is defined.

We focus on the region of C-H vibrations, located between 2500 and 3200 wavenumbers. Below 2500 wavenumbers there is a region where only a select few bonds are resonant, this region is also called the 'silent region'. At frequencies above 3200 wavenumbers, there are also very few resonant bonds, the most notable being the $\mathrm{O}-\mathrm{H}$ water bond [25]. Therefore the C-H vibrations can be considered to be isolated, since in most materials there are no nearby resonances which influence the vibrational response in the $\mathrm{C}-\mathrm{H}$ region, and we can fit this subsection of the vibrational response independently.

As an initial guess we start off with a number of resonances, typically 30 to 50 , which are spaced equally over the $\mathrm{C}-\mathrm{H}$ vibrational region. CMA-ES is subsequently used to find the optimal values for the linewidth, damping factor and center frequency of each resonance. The linewidth $\gamma$ is constrained between 3 and 50 wavenumbers and the center frequency of each resonance is allowed to be shifted up or down by a maximum of 4 times the spacing between adjacent resonances. In the case of 35 resonances spread over a 700 wavenumber region, this would correspond to a maximum center frequency shift of 80 wavenumbers for each resonance. For the optimization we use 20 parents and a population size of 40. An example of measured spontaneous Raman spectra and their corresponding fits is shown in figure 4.1. It can be seen that there is a good agreement between the spontaneous Raman scattering spectra and the obtained fits. The phase that corresponds to the fits is also shown in figure 4.1, as well as the relative strengths of the reso- 

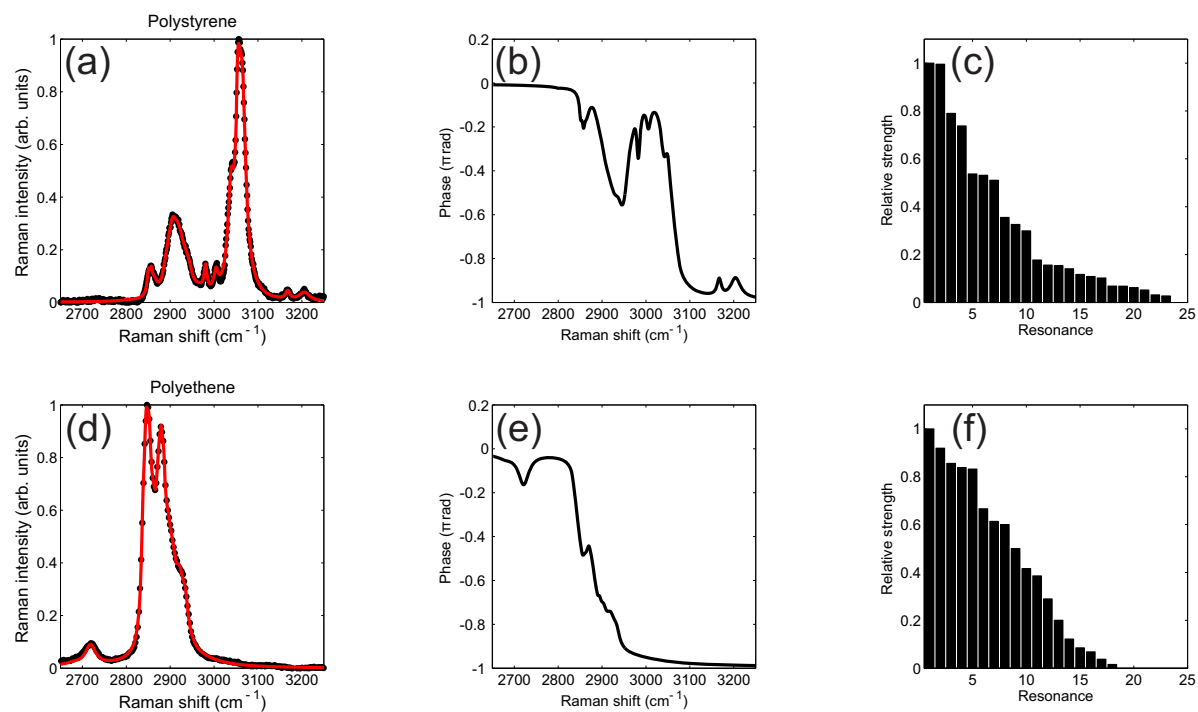

Figure 4.1: Spontaneous Raman spectra (black) and the imaginary part of the corresponding fitted function (red) for (a) polystyrene and (d) polyethene, and the retrieved phase response for (b) polystyrene and (e) polyethene. In $(c, f)$ the relative strengths of the different resonances used in the fits are shown.

nances used in the fit.

Since we use a mean squared error function as a fitness value, large discrepancies between the imaginary part of the fit and the spontaneous Raman spectrum are heavily penalized. The consequence is that the fitting of the strongest resonances is prioritized, as this constitutes the biggest increase in fitness value. If the center frequency of the fitted resonances is unconstrained, this will shift all these resonances to the strongest peaks in the Raman spectrum very early on in the optimization, leading to a slow convergence when fitting the weaker resonances later on. To avoid this effect, one would like to constrain the shift of the center frequency to be no more than the resonance spacing that the fit starts off with. This constraint requires a very small resonance spacing to accurately model complex Raman spectra however, corresponding to a large number of fitting parameters, which also slows down the convergence. The constraints on the center frequency shift and the number of resonances that are reported earlier in this chapter were empirically found to produce reasonable results. Other strategies, such as enforcing a minimum spacing between adjacent resonances, might solve this prob- 
lem as well.

The optimization typically converges in about 2000 generations, depending on the complexity of the vibrational spectrum that is being fitted. The parameters that are found by the CMA-ES also include resonances with very low amplitude, as typically not all initial resonances are required for an optimal fit. Any resonances with an amplitude smaller than $1 \%$ of the strongest resonance are considered to be of minimal influence and are discarded. Such weak resonances are almost indistinguishable from the noise in the spontaneous Raman spectra that we use, and as such one might be fitting noise instead of actual resonances by including them.

\subsubsection{Non-resonant background}

Using the spontaneous Raman spectra, we are able to fit the imaginary part of the complex vibrational response to the Raman data. Because the spontaneous Raman spectrum is proportional to the imaginary part of the vibrational response, any purely real contributions to the vibrational response will not show up in the spontaneous Raman spectrum. In CARS, the purely real contribution to the vibrational phase response shows up as a non-resonant background signal (see section 2.2.2). Since this non-resonant contribution cannot be obtained from the spontaneous Raman spectrum, it has to be estimated. It is possible to either add a non-resonant background that is constant with respect to frequency or a variable non-resonant background function to the obtained vibrational response model. We use a non-resonant background that has an amplitude of $20 \%$ of the maximum amplitude of the peak of the imaginary part of the resonant vibrational response and we consider the non-resonant background to be constant with respect to frequency. The complex vibrational response is subsequently constructed from the fitted resonances and the chosen non-resonant background function, as shown in figure 4.2. The vibrational response is found to be in good agreement with experimentally obtained values [30].

\subsection{Simulating the CARS response}

We use a time-invariant model, as described in section 2.5, where the CARS signal can be described by a series of convolutions between the input fields and a multiplication with the complex vibrational response (equation 4.4). 

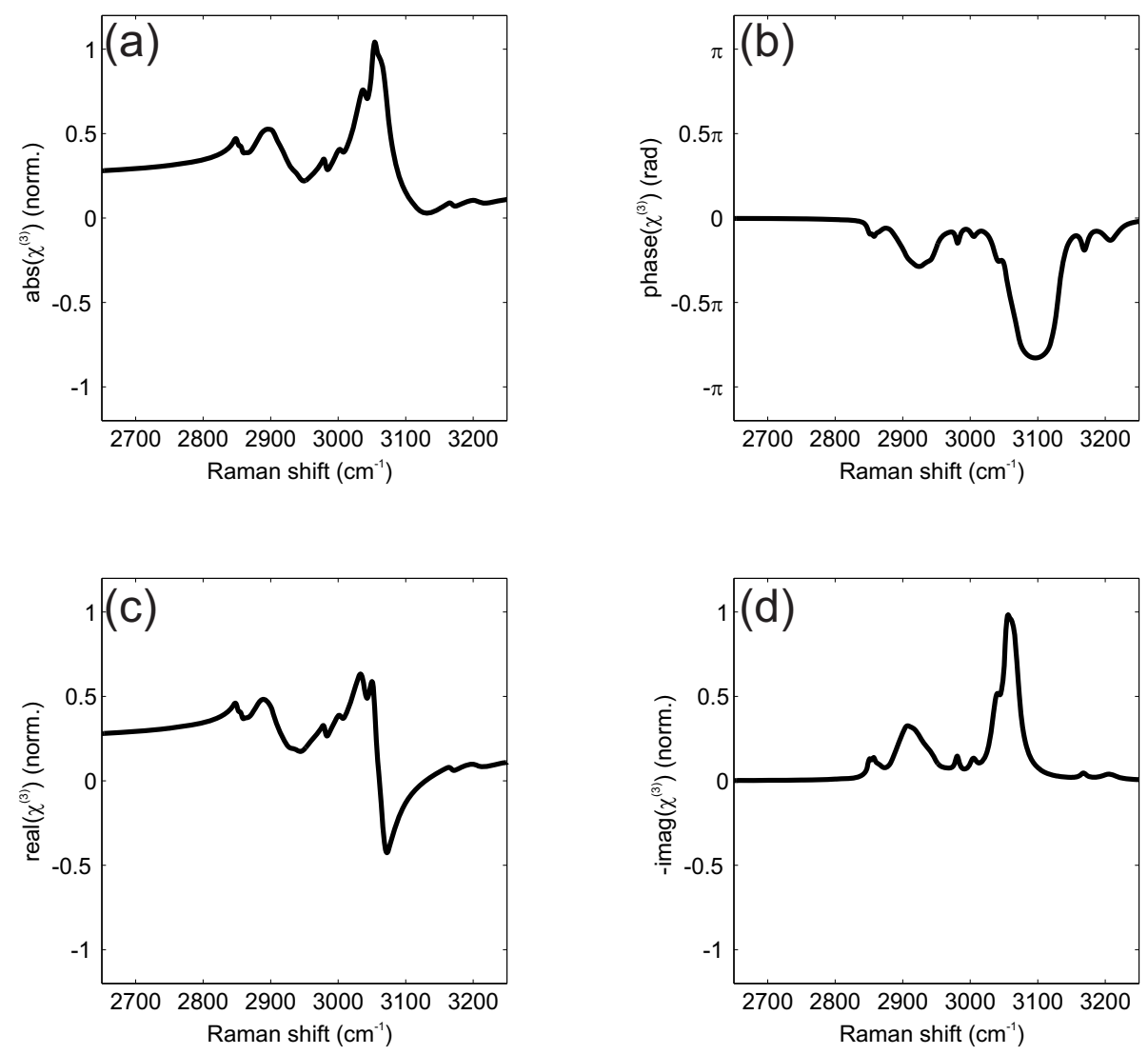

Figure 4.2: Molecular response of polystyrene with non-resonant background. (a) Absolute part of $\chi^{(3)}$, (b) phase of $\chi^{(3)}$, (c) real part of $\chi^{(3)}$, and (d) minus imaginary part of $\chi^{(3)}$. 


$$
\begin{array}{r}
I_{C A R S}(\omega) \propto \mid\left[\left(\left|E_{\text {pump }}(\omega)\right| e^{i \phi_{\text {pump }}(\omega)} \otimes\left|E_{\text {Stokes }}^{*}(\omega)\right| e^{-i \phi_{\text {Stokes }}(\omega)}\right) .\right. \\
\left.\chi^{(3)}(\omega)\right]\left.\otimes\left|E_{\text {probe }}(\omega)\right| e^{i \phi_{\text {probe }}(\omega)}\right|^{2}
\end{array}
$$

In the case of a CARS process with a broadband pump and probe pulse and a narrowband Stokes pulse, this equation can be simplified. Due to the very narrow bandwidth of the Stokes pulse, it is approximated by a delta function at the center frequency of the pulse. As a result, the only effect of the convolution with the Stokes pulse is a convolution with a delta function, which equates to a frequency shift of the pump pulse, equal in magnitude to the center frequency of the Stokes pulse. Equation 4.5 shows the resulting simplified equation for the generated CARS signal.

$$
\begin{array}{r}
I_{C A R S}(\omega) \propto \mid\left[\left|E_{\text {pump }}\left(\omega+\omega_{\text {Stokes }}\right)\right| e^{i \phi_{\text {pump }}\left(\omega+\omega_{\text {Stokes }}\right)} \cdot \chi^{(3)}(\omega)\right] \otimes \\
\left.\left|E_{\text {probe }}(\omega)\right| e^{i \phi_{\text {probe }}(\omega)}\right|^{2}
\end{array}
$$

Calculating the CARS signal is a computationally heavy process due to the convolution in equation 4.5. Because every point of the spectrum interacts with every other point, the complexity of a standard convolution is $O\left(N^{2}\right)$. Using the convolution theorem, this can be reduced with the help of fast Fourier transforms. By using a fast Fourier transform, multiplication, and inverse fast Fourier transform, the convolution can be calculated with $O\left(N \log _{2}(N)\right)$ complexity, which is a lot more efficient [67]. To use fast Fourier transforms, the spectra need to be defined over a number of points that equals $2^{N}$, where $\mathrm{N}$ is a natural number. Therefore the spectrum of the pump and probe pulse is defined over 4096 points and the corresponding phase profile is extended to the same 4096 points by cubic spline interpolation. The complex molecular response is also defined over 4096 points, with a $1 \mathrm{~cm}^{-1}$ resolution.

\subsection{Single compound optimization}

We can use the calculated CARS signal to determine the fitness (equation 4.6) in a closed loop optimization to find an optimal excitation 
phase. Our goal is to find an excitation phase that maximizes the amount of resonant CARS signal from a substance and simultaneously minimizes any purely non-resonant contributions.

To optimize only the resonant signal, the non-resonant contribution to the CARS signal needs to be discarded. Therefore we look at the difference in CARS signal generated by a phase profile $\phi(\omega)$ and the inverse phase profile $-\phi(\omega)$, which is free of non-resonant background, as shown in section 2.5.2. This CARS difference signal still contains some non-resonant contributions due to the homodyne mixing of the CARS process (see chapter 2.5), but since this term is dependent on the amount of resonant contribution to the CARS signal as well, it does not conflict with our optimization goal.

We apply CMA-ES to optimize the spectral excitation phase, where we use the aforementioned spectrally integrated CARS difference signal as fitness, as shown in equation 4.6.

$$
F(\phi(\omega))=\left|\sum_{\omega=\omega_{\min }}^{\omega_{\max }} I_{\phi}(\omega)-I_{-\phi}(\omega)\right|
$$

where $\mathrm{F}$ is the fitness, $I_{\phi}(\omega)$ is the CARS intensity resulting from the phase profile $\phi(\omega), I_{-\phi}(\omega)$ is the CARS intensity resulting from the inverse phase profile $-\phi(\omega)$, and $\omega_{\max }$ and $\omega_{\min }$ are the spectral limits between which the CARS spectrum is integrated.

We use a degenerate pump and probe pulse, centered at $12420 \mathrm{~cm}^{-1}$ $(805.2 \mathrm{~nm})$, with a bandwidth of $216 \mathrm{~cm}^{-1}(14 \mathrm{~nm})$. The Stokes pulse is assumed to be infinitely narrow with a center frequency of 9395.85 $\mathrm{cm}^{-1}(1064.3 \mathrm{~nm})$. The optimization of the phase profile is performed over 60 parameters. These parameters describe the phase and are evenly spaced along the frequency axis over a $574 \mathrm{~cm}^{-1}$ region centered around the center frequency of the pump and probe pulse. The phase profile is interpolated to a resolution of $1 \mathrm{~cm}^{-1}$ by cubic spline interpolation. The algorithm is run for 1000 generations with 20 parents and a population size of 40 per generation. The non-resonant background is assumed to be constant at $20 \%$ of the imaginary part of the main peak in the vibrational response. The results of an optimization for PMMA are shown in figure 4.3, along with the results of an optimization for polystyrene (PS). The fitness in figure 4.3 is normalized to the fitness of a pump pulse that matches the vibrational phase of the molecule. 
Figure 4.3 shows that the excitation phase profiles for maximum (difference) CARS signal for PMMA and polystyrene mainly follow the general shape of the molecular phase response. At high wavenumbers, the phase returns to 0 instead of $\pi$ due to the mixing between the resonant signal and the non-resonant background. Furthermore, it can be seen that the optimization converges in roughly 100 generations.

As a comparison for the amount of CARS signal generated, the optimal CARS difference signal is about an order of magnitude more intense than the CARS signal from a $15 \mathrm{ps}\left(1 \mathrm{~cm}^{-1}\right)$ pump and probe pulse, with the same pulse energy, focused on the main resonance of polystyrene. This comparison is based on a pure substance, with a constant non-resonant background of $20 \%$ of the maximum of the resonant vibrational response.

\subsection{Selective excitation}

\subsubsection{Multiple compound mixtures}

Besides optimizing the phase for maximum (difference) signal from a single compound, it is also possible to optimize for minimum (difference) signal. Combining these two ideas, we try to optimize the excitation phase for selective excitation of a single constituent in a mixture of compounds, by simultaneously optimizing for maximum signal from one compound and minimum signal from other compounds in the sample.

Here, we consider a mixture of five compounds, namely polystyrene, PMMA, polyethene, toluene, and ethanol. These five substances have strongly overlapping resonances in the region around $3000 \mathrm{~cm}^{-1}$, which is accessed by our combination of input pulses (pump-Stokes). The vibrational spectra of these compounds, which have been obtained from spontaneous Raman scattering spectra, as described in section 4.2.3, are shown in figure 4.4 .

Using CMA-ES, we find five different excitation phases, which selectively excite one of the resonant compounds, while minimizing the contributions from the other four compounds. As the fitness value, we use the difference signal of the compound that is being optimized minus two times the difference signal of the compounds that are being suppressed, as shown in equation 4.7. By subtracting two times the difference signal 

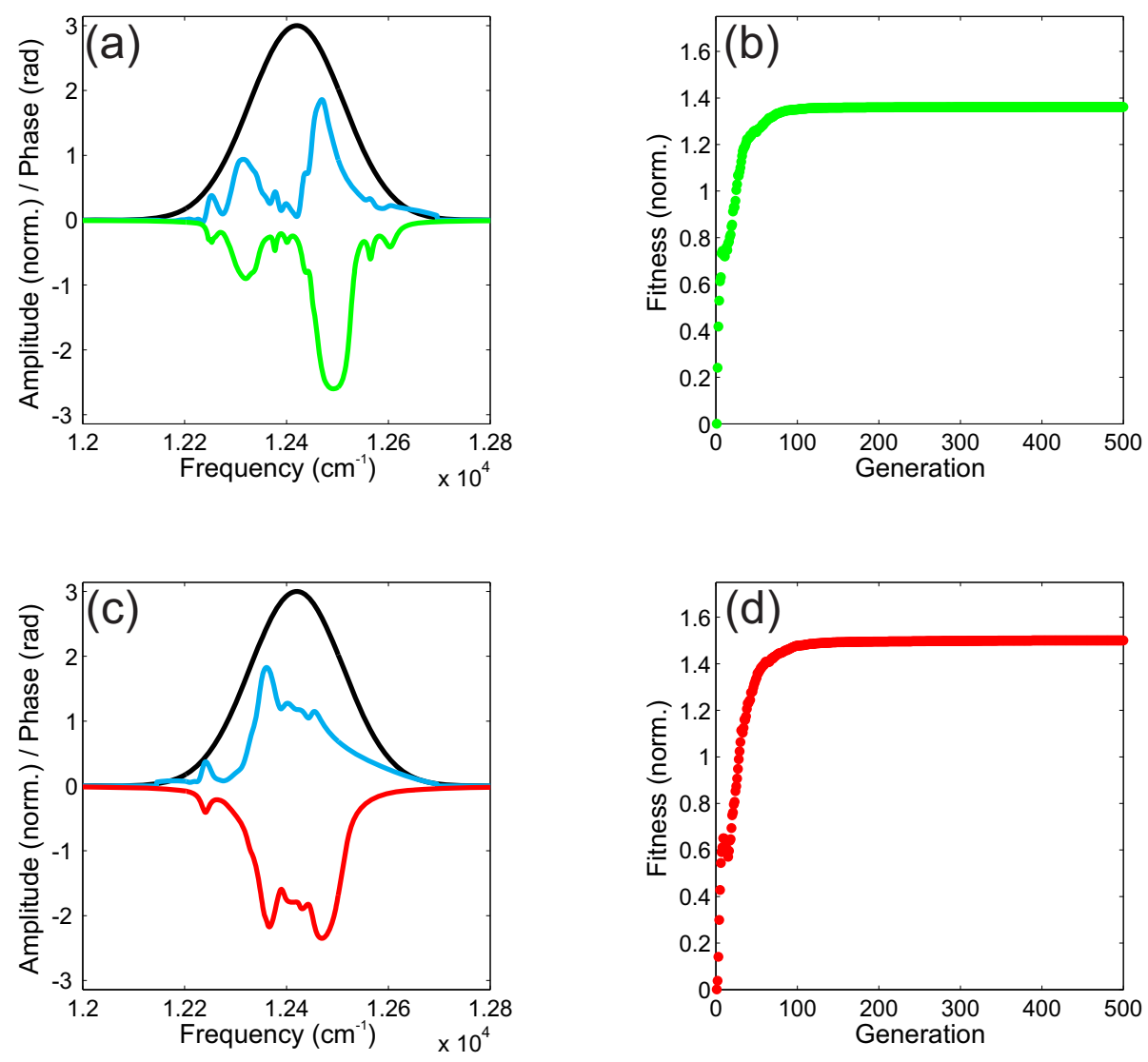

Figure 4.3: Numerical optimization of spectral excitation phase for PMMA and PS. (a,c) shows the intensity of the pump and probe pulse envelope (black) and the optimized spectral phase (cyan) for PS (a) and PMMA (c), the molecular phase (shifted by the Stokes frequency) is shown in green $(P S)$ or red (PMMA). (b,d) Mean fitness per generation for the optimization of PS (b) and PMMA (d) normalized to the fitness of a pump pulse with the vibrational phase of the molecule. 


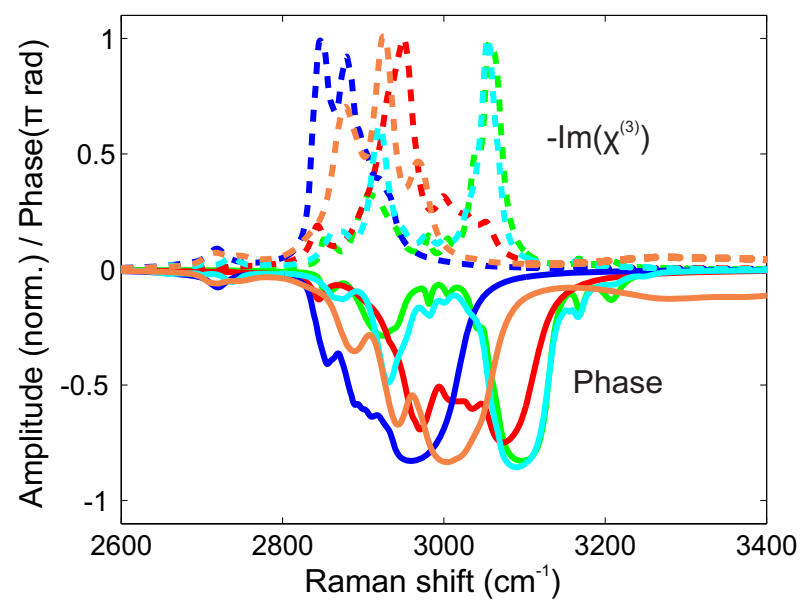

Figure 4.4: Vibrational spectra in the $\mathrm{C}-\mathrm{H}$ region for polystyrene (green), PMMA (red), polyethene (blue), toluene (cyan), and ethanol (orange), obtained from fits of spontaneous Raman scattering spectra. The dashed lines indicate the minus imaginary part of the $\chi^{(3)}$, which is proportional to the spontaneous Raman spectrum. The solid lines indicate the phase of the vibrational response of the molecules.

of the suppressed components, we penalize trivial solutions that equally increase the difference signal from both the component that is being optimized as well as a component that is being suppressed.

$$
\begin{array}{r}
F(\phi(\omega))=\Delta I_{\text {optim }}-2 \sum \Delta I_{\text {supp }}, \\
\Delta I=|I(\phi(\omega))-I(-\phi(\omega))|
\end{array}
$$

The pump (=probe) pulse is assumed to have a Gaussian spectral power distribution with a center frequency of $12400 \mathrm{~cm}^{-1}(806.5 \mathrm{~nm})$ and a full width at half-maximum (FWHM) of $400 \mathrm{~cm}^{-1}(26 \mathrm{~nm}, 37$ fs). The Stokes pulse is assumed to have a frequency of $9395.85 \mathrm{~cm}^{-1}$ $(1064.3 \mathrm{~nm})$ and an infinitesimally small bandwidth. For these simulations, the spectral phase profile is optimized by the CMA-ES on 40 points, divided evenly over $1021 \mathrm{~cm}^{-1}$. For each generation, 20 parents and a population size of 40 are used. The non-resonant background is assumed to be constant with a value of $20 \%$ of the main peak of the imaginary part of the vibrational response. 

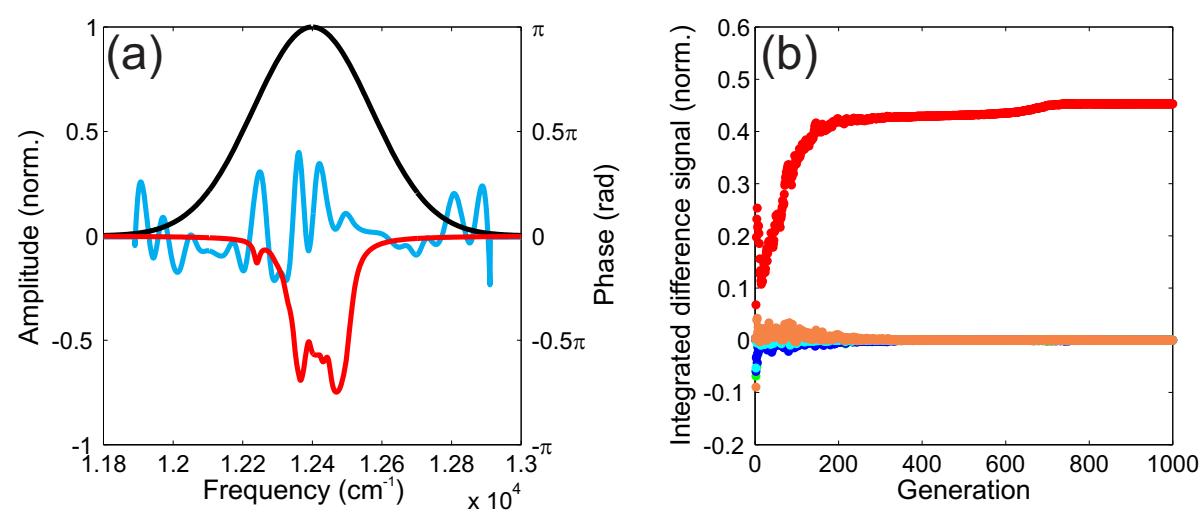

Figure 4.5: Selective excitation of PMMA in a sample of PMMA, polystyrene, polyethene, toluene, and ethanol. (a) shows the pump and probe intensity envelope (black), the molecular phase of PMMA (red) (shifted by the Stokes frequency), and the optimal excitation phase (cyan). (b) shows the difference intensity for PMMA (red), polystyrene (green), polyethene (blue), toluene (cyan), and ethanol (orange) as a function of generation of the evolutionary optimization, normalized to the difference intensity from PMMA for a pulse optimized for maximum signal from pure PMMA.

The results of the optimization for selective detection of PMMA in this mixture are shown in figure 4.5. Figure 4.5(b) shows the corresponding learning curve, showing how contributions from the other molecules are actively suppressed.

After 1000 generations, the CARS signal from PMMA in the mixture reaches a contrast ratio of 2200:1. The contrast ratio is defined by the ratio in CARS difference signal between the optimized compound and the other compounds in the sample, where the lowest ratio is used as the total contrast ratio. It can be seen from figure 4.5 that the optimal phase becomes more structured, to suppress the signal of the other resonant compounds. In contrast to figure 4.3, the optimal phase now deviates significantly from the molecular phase of PMMA. The phase profile in the wings of the pump and probe pulse varies significantly, because the optimization is influenced very little by these points and the phase at these wavelengths remains mostly random. Figure 4.5 also shows that the algorithm converges slower compared to a single compound optimization without suppression, which is to be expected due to the increased complexity of the optimization goal. The absolute amount of CARS 


\begin{tabular}{cccccc} 
& PS & PMMA & PE & Tol. & Eth. \\
\hline PS & 0.02 & $<3 * 10^{-4}$ & $<1 * 10^{-3}$ & $<5 * 10^{-4}$ & $<7 * 10^{-4}$ \\
\hline PMMA & $<1 * 10^{-4}$ & 0.45 & $<1 * 10^{-3}$ & $<2 * 10^{-4}$ & $<7 * 10^{-4}$ \\
\hline PE & $<1 * 10^{-4}$ & $<2 * 10^{-4}$ & 0.44 & $<2 * 10^{-4}$ & $<7 * 10^{-4}$ \\
\hline Tol. & $<1 * 10^{-4}$ & $<2 * 10^{-4}$ & $<1 * 10^{-3}$ & 0.08 & $<7 * 10^{-4}$ \\
\hline Eth. & $<2 * 10^{-4}$ & $<3 * 10^{-4}$ & $<1 * 10^{-3}$ & $<3 * 10^{-4}$ & 0.27 \\
\hline Ratio & $132: 1$ & $2200: 1$ & $470: 1$ & $168: 1$ & $415: 1$
\end{tabular}

Table 4.1: CARS signals and contrast ratios for selective excitation of a single compound in a mixture of 5 resonant compounds. The highlighted cells indicate the optimized compound and the cells of that column show the amount of integrated difference CARS signal normalized to that obtained from a pure sample optimization.

difference signal from the PMMA has decreased by $55 \%$ compared to the optimization of a pure PMMA sample (without suppression of other compounds). The results and contrast ratios for all five substances can be found in table 4.1.

In table 4.1 we can see that selective imaging is possible for all five resonant compounds in the sample, with high contrast ratios, ranging from 100:1 to 2000:1. Furthermore, it can be seen that the contrast and total signal is lowest for polystyrene and toluene. These two molecules have very similar chemical structures, and hence the vibrational response is very similar, making it more difficult to differentiate between the two.

It should be noted that the contrast ratios in table 4.1 refer to signal levels of pure substances. The contrast ratio might be lower if multiple resonant compounds are present in the focal volume simultaneously. This potential decrease in contrast ratio is due to homodyne mixing of the CARS signals, and will be described in detail in section 4.5.2.

\subsubsection{Influence of homodyne mixing}

In many samples, there are multiple components present in the focal volume simultaneously. The CARS signals from these different components are mixed and interfere, which influences the collected CARS signal in a way that deviates from linear addition. Therefore, it is important not only to look at the response for a pure substance, but also to consider this homodyne mixing of the CARS fields from different components. To study the effect of homodyne mixing on our optimization approach we 
use a mixture of three resonant components: polystyrene (PS), PMMA, and polyethene $(\mathrm{PE})$.

Using the optimization approach described in section 4.5.1, three different excitation phases are calculated for selective excitation of each of the three compounds. For the optimization, the same parameters as in section 4.5.1 are used. Subsequently, we calculate the effect of these phase shapes on different combinations of concentrations of the resonant compounds in the focal volume. The CARS signal for different mixtures within the focal volume is shown in figure $4.6(\mathrm{a}-\mathrm{c})$ in the form of a ternary plot. Each of these three subfigures shows the results for one of the optimal phase profiles.

It can be seen from figure 4.6 that there is a close-to-linear dependence between the CARS difference signal and the concentration for most mixtures, except near the region where there is less than a few percent of the substance that was optimized in the focal volume. For the optimized profiles for PMMA and polystyrene in figures 4.6(d) and 4.6(e), there is a (green) band of mixing combinations, where the signal from small amounts of the substance of interest is suppressed due to mixing with the signal of the suppressed substances (figure 4.6(d,e)).

Furthermore, mixing between the signals from both suppressed components can produce a stronger signal than both components would produce by themselves, leading to an overestimation of the concentration. It can be seen in figure 4.6(d) that in the case of the PMMA-optimized profiles, mixing between the two suppressed components generates a negative difference signal (causing the dip for PMMA in figure 4.6(h)), which can be easily distinguished from the positive difference signal that the component of interest generates. In the case of the polystyrene and polyethene optimized profiles (figure 4.6(e,f)), there is a positive difference signal for mixing between PMMA and polystyrene however. Both these effects limit the obtainable selectivity.

\subsubsection{Robustness to noise}

The effect of (phase) noise on the optimization is calculated to test the robustness of the optimization approach to phase fluctuations in the spatial light modulator. We simulate the effect of noise by adding random noise to each trial phase profile in the optimization, such that 
PMMA

(a)


(b)

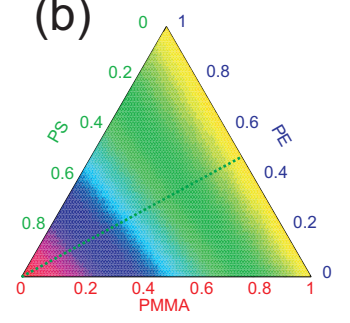

(e)

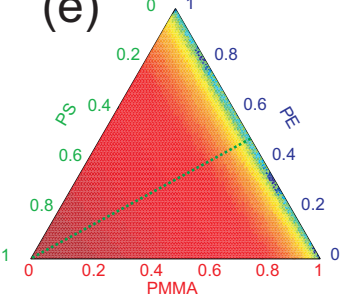

Polyethene
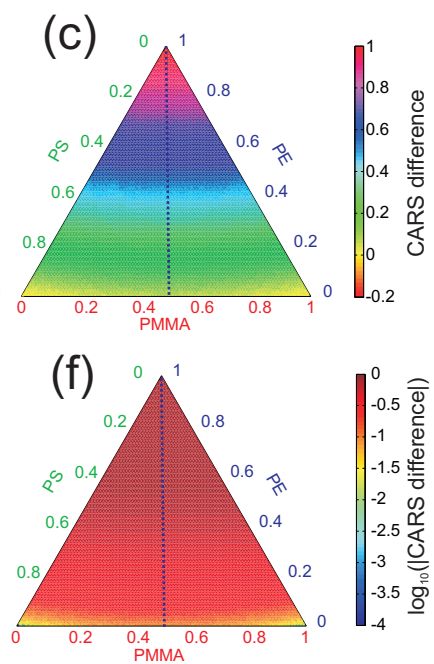



Figure 4.6: $C A R S$ difference signal for mixtures of PMMA, polystyrene and polyethene in the focal volume for the selective excitation phase of (a) PMMA, (b) polystyrene, and (c) polyethene. Panels (d-f) show the $\log _{10}$ of the absolute value of $(a-c)$. In $(g)$, the signal dependence is shown along slices in the ternary plot (dashed lines in $(a-c)$ ), where the relative concentration of the optimized component (red for PMMA, green for polystyrene, blue for polyethene) goes from 1 to 0, with a 50\%/50\% ratio between the other two components. The black dashed line in $(g, h)$ indicates an ideal linear dependence. (h) The dependence of the signal as in $(g)$, on a $\log _{10} / \log _{10}$ scale over 4 orders of magnitude. 


\begin{tabular}{ccc} 
Noise level $(\sigma)$ & Contrast ratio & Norm. CARS signal \\
\hline 0 & $2200: 1$ & 1 \\
\hline $0.01 \pi$ & $160: 1$ & 0.94 \\
\hline $0.02 \pi$ & $120: 1$ & 0.82 \\
\hline $0.05 \pi$ & $47: 1$ & 0.80 \\
\hline $0.1 \pi$ & $19: 1$ & 0.75 \\
\hline $0.2 \pi$ & $10: 1$ & 0.39 \\
\hline $0.3 \pi$ & $5: 1$ & 0.03 \\
\hline
\end{tabular}

Table 4.2: $C A R S$ signal and contrast ratios for selective excitation of PMMA in a sample containing PMMA, polystyrene, polyethene, toluene, and ethanol, in the presence of various levels of noise. The normalized signal is relative to the signal obtained in the case of no noise.

$\phi_{\text {total }}=\phi_{\text {orig }}+\phi_{\text {noise }}(\mu, \sigma)$, where $\phi_{\text {total }}$ is the phase profile with noise, $\phi_{\text {orig }}$ is the original noiseless phase profile and $\phi_{\text {noise }}$ is a random noise function with a normal distribution with mean $\mu=0$ and variance $\sigma$. The results for selective excitation of PMMA in a mixture of PMMA, polystyrene, polyethene, toluene, and ethanol are presented in table 4.2.

Table 4.2 shows that the presence of a few percent of (phase) noise has a large influence on the optimization. At low levels of noise it is primarily the contrast ratio that is reduced, as the noise makes it increasingly difficult to suppress the signal from unwanted compounds. At higher noise levels $(\sigma \geq 0.1 \pi)$ the absolute signal from the optimized compound also starts to decrease significantly. It should be noted however that even at large amounts of noise, the evolutionary algorithm still converges to a solution, providing contrast between the optimized compound and the suppressed compounds.

The optimized phase profiles for various levels of phase noise are shown in figure 4.7. From figure 4.7 it can be seen that the general shape of the optimal phase profile remains largely unchanged in the center of the pump pulse. In the wings of the pump pulse the phase profile deviates due to the noise.

\subsubsection{Optimization dimensionality}

To estimate the required resolution of phase shaping, which determines the required dimensionality of the optimization, we investigate the effect 

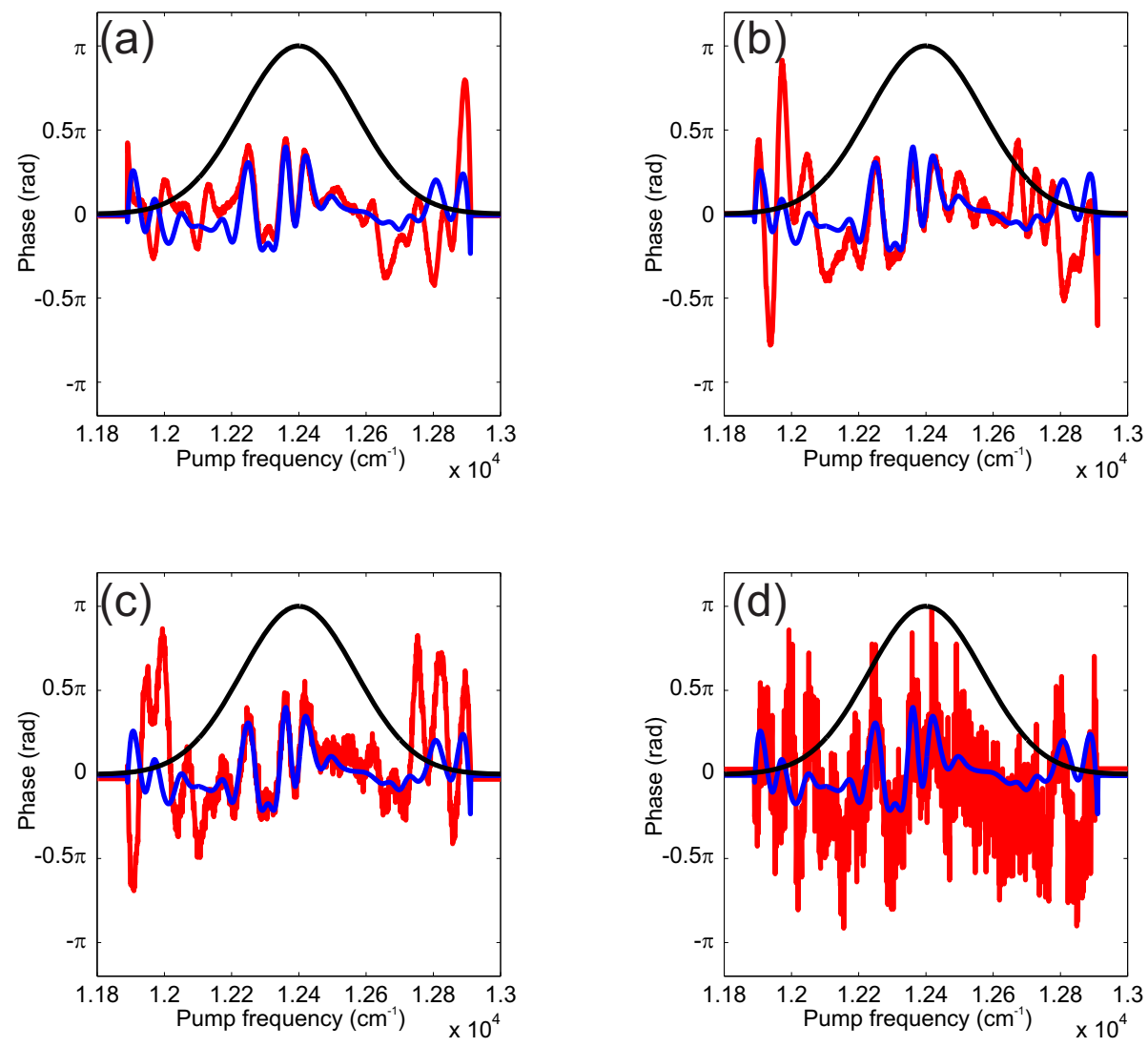

Figure 4.7: Phase profiles (red) for selective excitation of PMMA in a

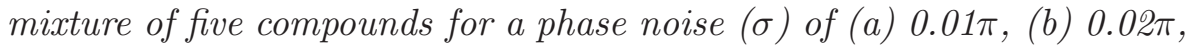
(c) $0.05 \pi$, and (d) $0.2 \pi$ radians. The intensity spectrum of the pump pulse is shown in black, and the optimal phase in the case of no phase noise is shown in blue. 


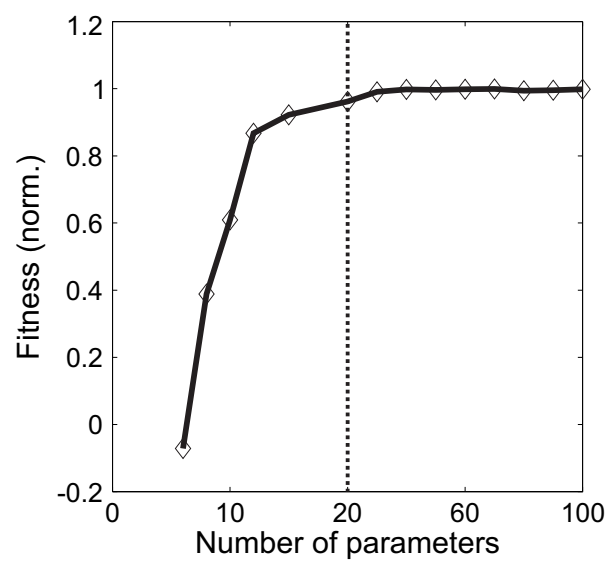

Figure 4.8: Fitness for selective excitation of PMMA (solid) as a function of number of parameters that the pulse is optimized with, normalized to the maximum obtained fitness. Note the different scaling of the horizontal axis on both sides of the dashed line.

of changing the number of parameters used to describe the phase profile of the pump and probe pulse. In this case, we look at the optimization of selective excitation of PMMA in a mixture of PMMA, polystyrene, and polyethene. The number of parameters used to describe the phase function of the excitation pulse is varied between 6 and 100, the other parameters of the CMA-ES are equal to those used in section 4.5.1. The fitness of the optimized phase profile, as defined in equation 4.7 and normalized to the maximum obtained fitness, is plotted in figure 4.8.

It can be seen from figure 4.8 that the minimum number of parameters required to accurately model the optimal phase function $(\geq 99 \%$ of maximum fitness) is 40 . This corresponds to a resolution in the shaping of about $25 \mathrm{~cm}^{-1}( \pm 1.5 \mathrm{~nm})$. A higher number of parameters does not significantly increase the amount of obtained signal or the contrast ratio. The number of elements on the phase shaper used in an experimental optimization has to be higher, so that the applied phase function matches the smooth interpolation between the parameters of the numerically optimized phase function. If we consider zero crosstalk between elements, at least 75 elements are required to obtain a fitness value higher than $90 \%$ of the fitness value for the cubic spline interpolated phase pattern, as shown in figure 4.9 .

Further improvements in terms of number of parameters and optim- 


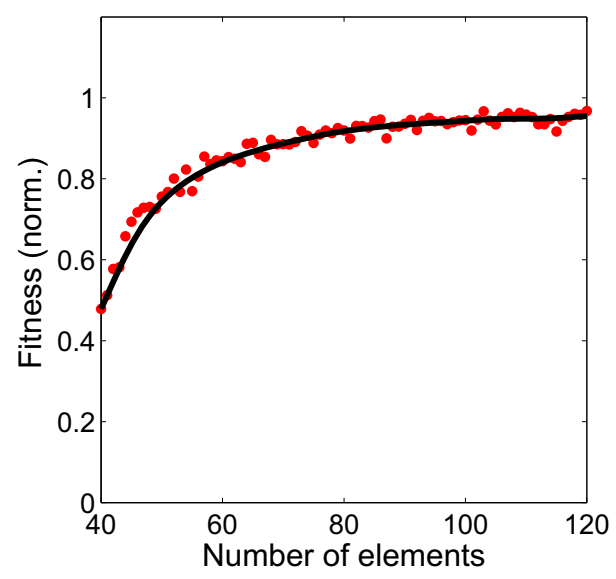

Figure 4.9: Fitness (red) in the case of no crosstalk between elements as a function of number of elements, normalized to the maximum fitness for a cubic spline interpolated phase pattern. The black line is a 10-point moving window average over the data points.

ization speed might be obtained by tailoring the basis set of parameters that define the phase function. As will be shown in section 4.6, the solution landscape around the optimal point can be optimized using Kautz functions as a basis set.

\subsection{Solution landscapes}

\subsubsection{Introduction}

A key question regarding shaped CARS experiments in general, and for optimizations in particular, is the shape and the topology of the solution space in which each point represents a phase function and its associated fitness. Ideally, the contour lines are convex, connected (no local optima), and without nonlinear shear (relative rotation) [68].

Theoretically, a shaped CARS problem can have an infinite dimensional solution landscape or functional space. We refer to this space as the global solution landscape. By modeling the spectral phase with a specific basis set, such as polynomials, a specific subset of the landscape is selected. We refer to such a subset using the term solution landscape. These landscapes can have similar or different properties compared to each other and compared to the global landscape. Selecting a suitable basis set with a well-behaved solution landscape can improve optimiza- 
tion speed and obtained fitness.

The work presented in section 4.6 was performed in close collaboration with Dr. Aliakbar Jafarpour.

\subsubsection{Optimization parameters}

To study the effect of different basis sets on the solution landscape, we consider a CARS process in bulk polystyrene. A constant non-resonant background with an amplitude equal to $12 \%$ of the main resonance of polystyrene (at $3052 \mathrm{~cm}^{-1}$ ) is used in our model. The spectra of the pump and the Stokes fields are centered at $12500 \mathrm{~cm}^{-1}$ and $9396 \mathrm{~cm}^{-1}$ (800 $\mathrm{nm}$ and $1064 \mathrm{~nm}$ ), respectively. The pump spectrum has a fullwidth at half maximum (FWHM) bandwidth of $400 \mathrm{~cm}^{-1}(25.6 \mathrm{~nm})$, and the Stokes pulse has a negligible bandwidth and is modeled by a Dirac delta function in the frequency domain.

CMA-ES is used to optimize the spectral phase of the pump (=probe) pulse for maximum difference signal from polystyrene, using a population size of 40 and 20 parents per generation. The phase of the pump and probe pulse is modeled on 80 points divided evenly over a $1021 \mathrm{~cm}^{-1}$ region. The values of the pump phase function at these 80 points are calculated by the optimization algorithm, and subsequently extended to 1021 points $\left(1 \mathrm{~cm}^{-1}\right.$ resolution) by cubic spline interpolation. The simulations in section 4.6 are performed using a model of the polystyrene vibrational response that uses three resonances, as shown in figure 4.10.

The fitness for a phase profile is defined as the difference signal generated by a pulse with that phase profile, divided by the difference signal generated by a pulse with a phase profile that matches the vibrational response of the molecule, as shown in equation 4.8. The highest fitness that was obtained is 2.048 .

$$
\begin{aligned}
F(\phi) & =\frac{\Delta I_{\phi}}{\Delta I_{\phi_{\text {mol }}}}, \\
\Delta I_{\phi} & =I_{\phi}-I_{-\phi}
\end{aligned}
$$




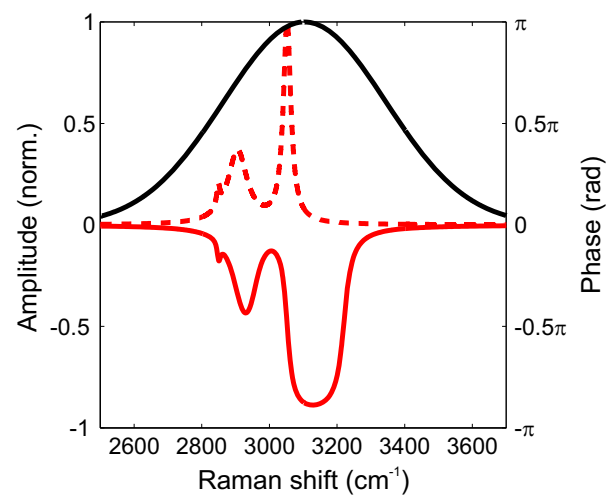

Figure 4.10: Pump pulse amplitude spectrum (black), shifted by the Stokes frequency, and the vibrational model of polystyrene (red) used in section 4.6, showing the minus imaginary part of the $\chi^{(3)}$ (dashed) and the vibrational phase (solid).

\subsubsection{Optimization landscapes}

Once the solution to the optimization problem is known, the landscapes around this point can be calculated by considering the phase function $\phi=\phi_{\text {opt }}+\phi_{\text {excess }}$, where $\phi_{\text {opt }}$ denotes the optimal phase profile and $\phi_{\text {excess }}$ is an excess phase profile superimposed on $\phi_{\text {opt }}$, the parameters of which are scanned.

While polynomials are successfully used to model the phase shifts associated with the tail of a resonance (as in material dispersion), they are not expected to be that efficient in modeling the sharp features and the flat regions associated with a Lorentzian resonance, simultaneously. Hence, in addition to polynomials, we also consider the alternative basis sets of rational functions [69]. A rational function is one that can be written as the ratio of two polynomials, such as a sum of some Lorentzian functions.

As a truncated Laurent series, a rational function can be useful in modeling a function without a Taylor series, or one requiring many orders of Taylor series and thereby enhancing numerical errors. Here, we use a rational Kautz basis, which comprises alternatively even and odd functions in the Laplace (complex frequency) domain, as shown in equations 4.10 and 4.11 . 

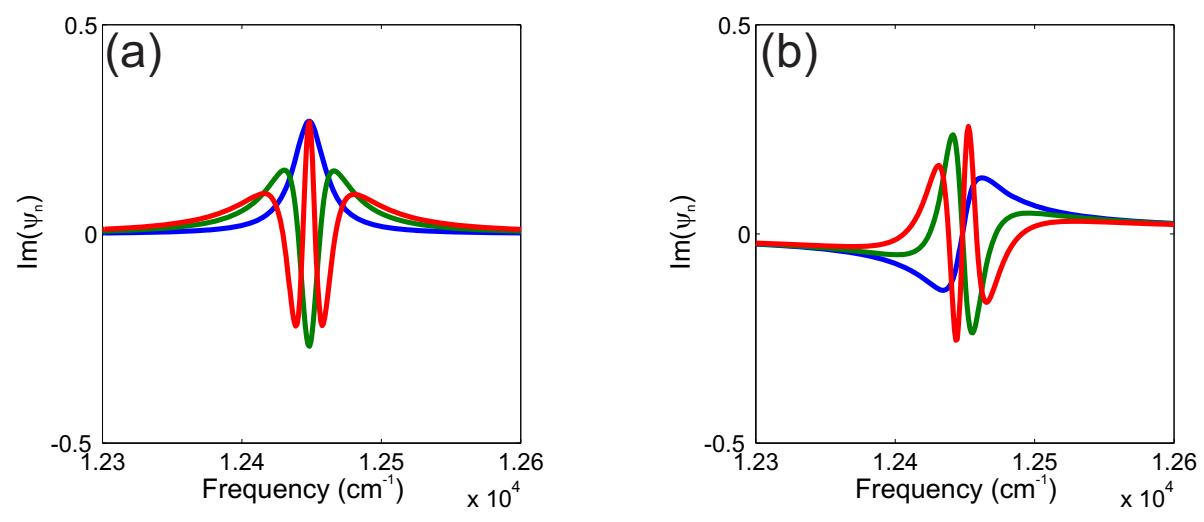

Figure 4.11: Imaginary part of the first six orders of the Kautz functions. (a) Zero (blue), second (green), and fourth (red) order. (b) First (blue), third (green), and fifth (red) order.

$$
\begin{aligned}
\psi_{2 n}(s) & =\frac{(2 b c)^{0.5}}{s^{2}+b s+c} \eta^{n} \\
\psi_{2 n+1}(s) & =\frac{(2 b)^{0.5} s}{s^{2}+b s+c} \eta^{n} \\
\eta & =\frac{s^{2}-b s+c}{s^{2}+b s+c}
\end{aligned}
$$

The time domain counterparts (inverse Laplace transforms) of the functions $\left\{\psi_{n}\right\}$ form a complete orthonormal set $\left\{\psi_{n}^{\prime}\right\}$ [70]. The complex radian frequency $s$ is related to the vibrational radian frequency $\omega$ as $s=i \omega$, where $i$ is the imaginary unit. The two real parameters $b$ and $c$ scale all orders of this basis set. They are chosen here as $b=27.51811$ and $c=9.3169 * 10^{6}$, to model the resonance frequency and the damping factor of the strongest resonance of polystyrene, centered at $3052 \mathrm{~cm}^{-1}$. There are different ways to derive a real function for the expansion of spectral phase from the complex Kautz basis functions. Here, we use the imaginary parts of the Kautz functions to obtain a real-valued function, as shown in figure 4.11. 

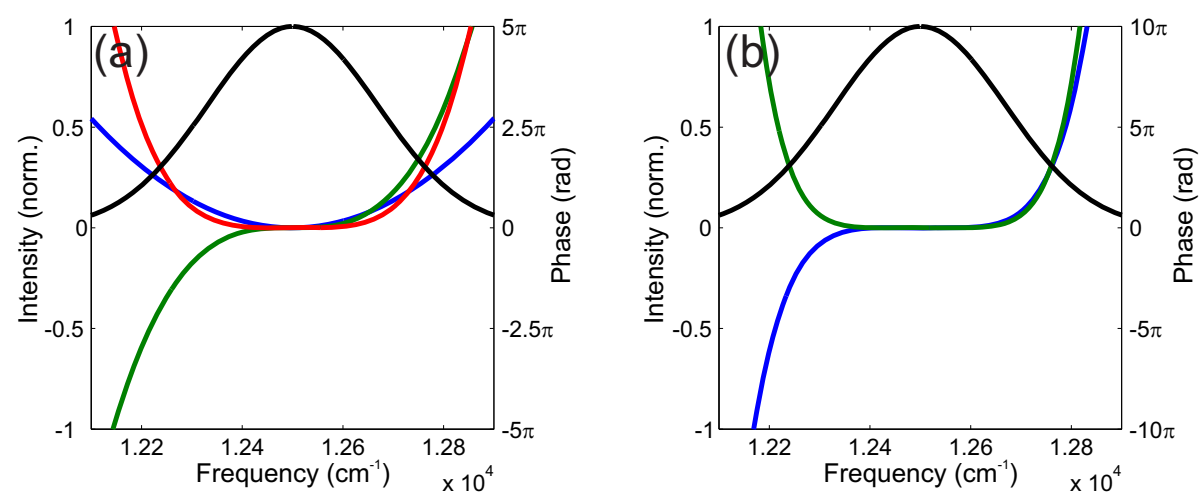

Figure 4.12: Pump pulse intensity spectrum (black) and second to sixth order polynomial functions. (a) Second (blue), third (green), and fourth (red) order. (b) Fifth (blue), and sixth (green) order.

\section{D Landscapes}

The effect of introducing a polynomial phase of $\phi^{(n)}(\omega)=\phi_{n}(\omega-$ $\left.\omega_{0}\right)^{n} / n$ !, where $\omega$ and $\omega_{0}$ denote the pump frequency and the pump center frequency respectively, and scanning the coefficient $\phi_{n}$ for different values of $2 \leq n \leq 6$ is shown in figure 4.13(a). As the phase order increases, the magnitudes of the slopes and curvatures increase at the origin and decrease at the tails of the curves, as can be seen in figure 4.13(a). The slight asymmetry of the $n=2$ case is partially attributed to a small interpolation error in the estimation of the optimal phase. The maximum values of individual polynomial phase terms over $1021 \mathrm{~cm}^{-1}$ are $\phi_{2, \max }=3 * 10^{3} \mathrm{fs}^{2}, \phi_{3, \max }=3.1 * 10^{5} \mathrm{fs}^{3}, \phi_{4, \max }=1.9 * 10^{7} \mathrm{fs}^{4}$, $\phi_{5, \max }=4 * 10^{9} f s^{5}$, and $\phi_{6, \max }=5 * 10^{11} f s^{6}$. These maximum values indicate the limits of the axes in figure 4.14 , which run from $-\phi_{n, \max }$ to $\phi_{n, \max }$. The phase functions corresponding to these maximum values are shown in figure 4.12 .

The 1D Kautz fitness landscapes corresponding to the first five orders $0 \leq n \leq 4$ are shown in figure 4.13(b). Contrary to the case of polynomials, these landscapes feature similar behaviors close to the origin and only start to deviate from each other, where the fitness drops to almost $25 \%$ of the maximum fitness. They also feature local optima for larger values of the coefficients $\phi_{n, \text { Kaut } z}$. 

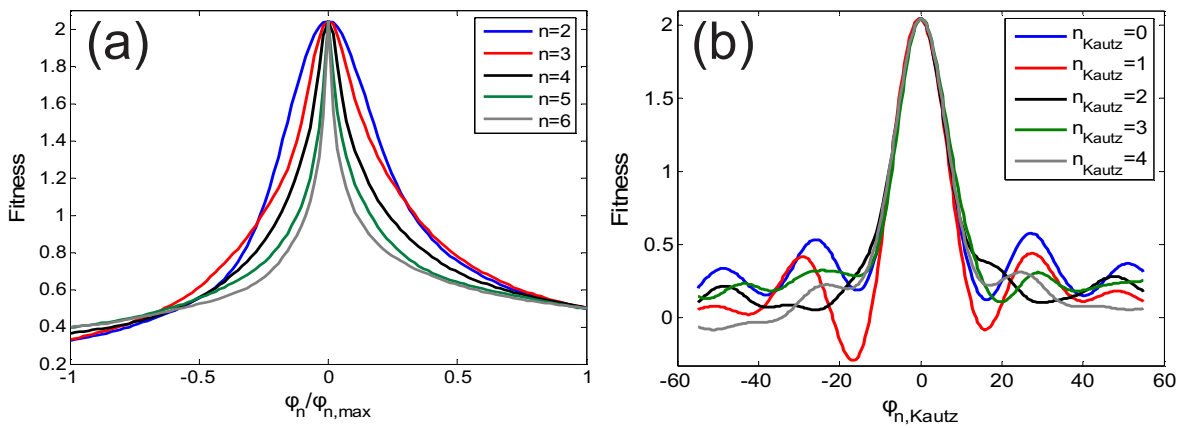

Figure 4.13: $1 D$ landscapes around the optimal point with (a) polynomials and (b) Kautz bases.

\section{D Landscapes}

Instead of scanning a single order, we can visualize the effect of adding two different orders simultaneously. The effect of the simultaneous presence of two polynomial orders in the spectral phase on the landscape is shown in figure 4.14. The two upper rows correspond to phase orders with opposite parities (one even, one odd), and the bottom row corresponds to phase orders with similar parities (both even or both odd). As the phase orders increase, the convexity of the contour lines decreases. The patterns associated with similar-parity orders feature a reduced symmetry, compared to quasi-symmetric patterns of oppositeparity orders. These symmetries are very similar to the $2 \mathrm{D}$ polynomial landscapes of second harmonic generation [68, 71].

The effect of the simultaneous presence of two Kautz functions in the spectral phase on the landscape is shown in figure 4.15. The two upper rows correspond to phase orders with opposite parities, and the bottom row corresponds to phase orders with similar parities. Similar to the case of polynomials, shown in figure 4.14, the Kautz landscapes with similar parities have different symmetries and their contours are extended along a line with a negative slope. Similar parities show that two orders with the same sign enhance each other, whereas the same orders with opposite signs partially cancel each other out.

Similar to the case of 1D Kautz landscapes, all 2D Kautz landscapes feature a bell-type pattern (red hot spot) at the origin. If the values of the coefficients are limited to this range, the landscapes have convex contour lines and no local optima, down to smaller values compared to 


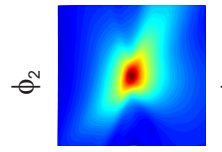

$\phi_{3}$

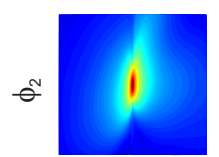

$\phi_{5}$

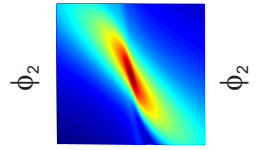

$\phi_{4}$

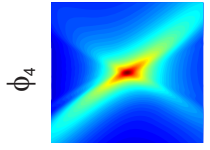

$\phi_{3}$

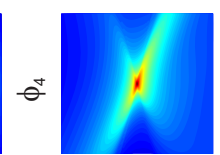

$\phi_{5}$

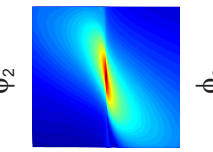

$\phi_{6}$

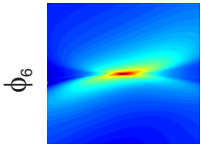

$\phi_{3}$

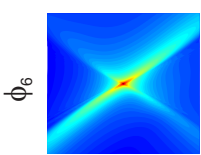

$\phi_{5}$

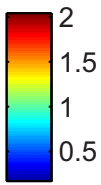

Figure 4.14: 2D landscapes around the optimal point with polynomials. (Top row) Even orders of 2, 4, and 6 with the third order. (Middle row) Even orders of 2, 4, and 6 with the fifth order. (Bottom row) Orders with similar parities: 2-4, 2-6, 3-5, and 4-6.

polynomial landscapes. Note that the considerable overlap of the 1D Kautz landscapes and the overlap of 1D polynomial landscapes at the peaks and also at the tails (figure 4.13) imply reasonably comparable ranges of different phase orders. As such, the elongation of a $2 \mathrm{D}$ contour line implies a property of the 2D landscape, and not incomparable ranges of phase terms.

The 2D landscapes corresponding to rational functions (figure 4.15) have the advantage of featuring convex and connected contours down to relatively small values of fitness ( $27 \%$ of the maximum fitness for the first two Kautz orders) in the vicinity of the optimal phase.

\section{Quantitative comparison of landscapes}

We consider two figures of merit that can be used for quantitative comparison of different basis sets, namely the isoperimetric coefficient (IPC) and the convex hull quotient (CHQ). In an ideal optimization landscape the contours are symmetric, isotropic, convex (see figure 4.16), connected, and without relative rotation. We can look at the isoperimetric coefficient by considering these contours as a closed string. A closed string will enclose the largest area when it is stretched uniformly to form 


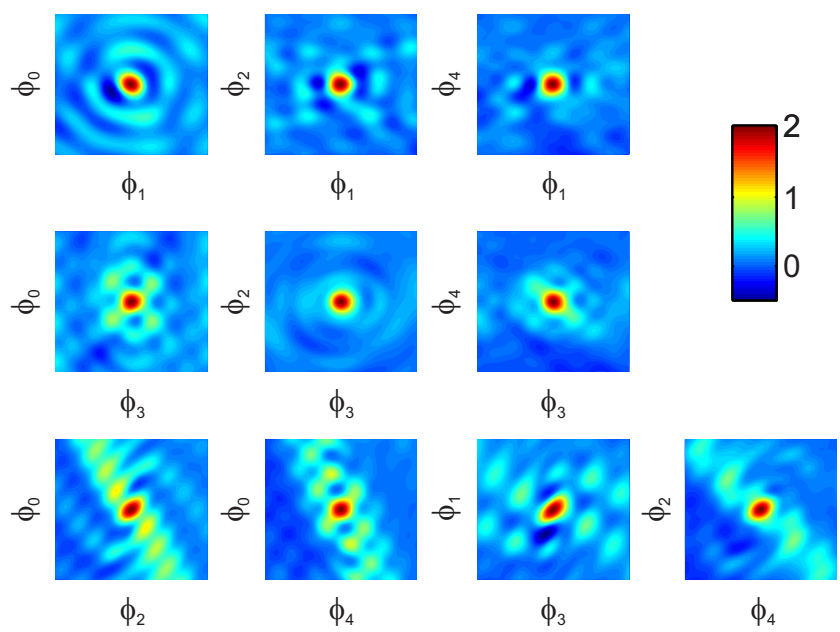

Figure 4.15: $2 D$ landscapes around the optimal point with Kautz functions. (Top row) Even orders of 0, 2, and 4 with the first order. (Middle row) Even orders of 0, 2, and 4 with the third order. (Bottom row) Orders with similar parities: 0-2, 0-4, 1-3, and 2-4. The landscapes are



a circle. This intuitive property, referred to as the isoperimetric inequality, is the basis of our approach. Also note that any concave shape can be modified towards a convex shape with the same circumference, but a larger area, by 'flipping the dents' (see figure 4.16(c)). If $\mathrm{P}$ and A denote the circumference and the area of a closed shape, the ranking factor $I P Q=4 \pi A / P^{2}$ (referred to as the isoperimetric quotient) ranks convex shapes on top of 'comparable' concave ones (see figure 4.16(c)) and a circle on top of all convex shapes; which is exactly what we are looking for. The IPQ can also be expressed in three- and higher-dimensional spaces, where visual inspection is difficult or even impossible. While the IPQ is a useful figure of merit to evaluate the convex shape and the apparent ellipticity of a contour, it can yield similar values for a concave contour and an elongated convex one. A complementary figure of merit is one that only differentiates between convex and concave contours with no sensitivity to the elongation of convex contours.

When a stretched elastic band encompassing an object is released, it is (partially) attached to a cross section of the object. The resulting shape of the released band is referred to as the convex hull (minimal convex set) of the cross section. If the cross section is convex, the convex 
(a)



(b)

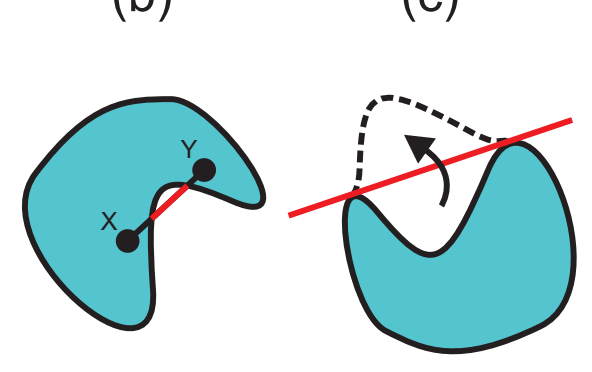

(c) (d)

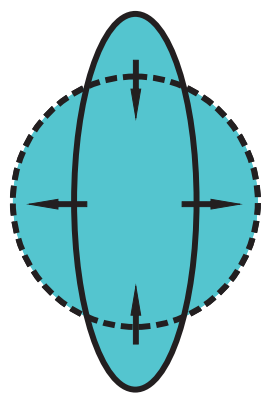

Figure 4.16: (a) A convex curve; the line segment connecting any two arbitrary points within the enclosed area will lie entirely within the enclosed area. (b) A concave curve; the line segment connecting at least one pair of points within the enclosed area will lie partially outside the enclosed area. (c) By flipping a dent, it is possible to convert a concave shape to a convex one with the same circumference, but a larger area. (d) Among convex patterns with the same circumference, a circle encloses the largest area.

hull is the cross section itself. The difference between the areas enclosed by a contour and its convex hull is a measure of deviation from a convex shape. We define a second figure of merit, referred to as Convex Hull Quotient (CHQ), to be the ratio of the areas enclosed by a contour and its convex hull. CHQ is a figure of merit complementary to the IPQ. For CHQ values (considerably) smaller than unity, the contours have (considerable) deviation from a convex shape. If the CHQ is (close to) one, then the IPQ is (mainly) a measure of elongation of a convex contour.

We compare the landscape for second- and third-order polynomials to the landscape of the 0th and 1st order Kautz functions using the IPQ and CHQ (see figure 4.17). The number of data points along each coordinate is chosen to be 200 in order to have a reasonably small numerical error in discretization of contours with small areas. The first analyzed contour corresponds to $88 \%$ of the maximum fitness, which has been discretized with 35 points along the periphery. The last analyzed contour corresponds to $39 \%$ of the maximum fitness, and has been discretized with 245 points. It corresponds to the lowest fitness, for which the contour in the polynomial landscape is completely accommodated in the chosen range of the landscape. From figure 4.17 it can be seen that the contours in the polynomial landscape deviate more from a convex 

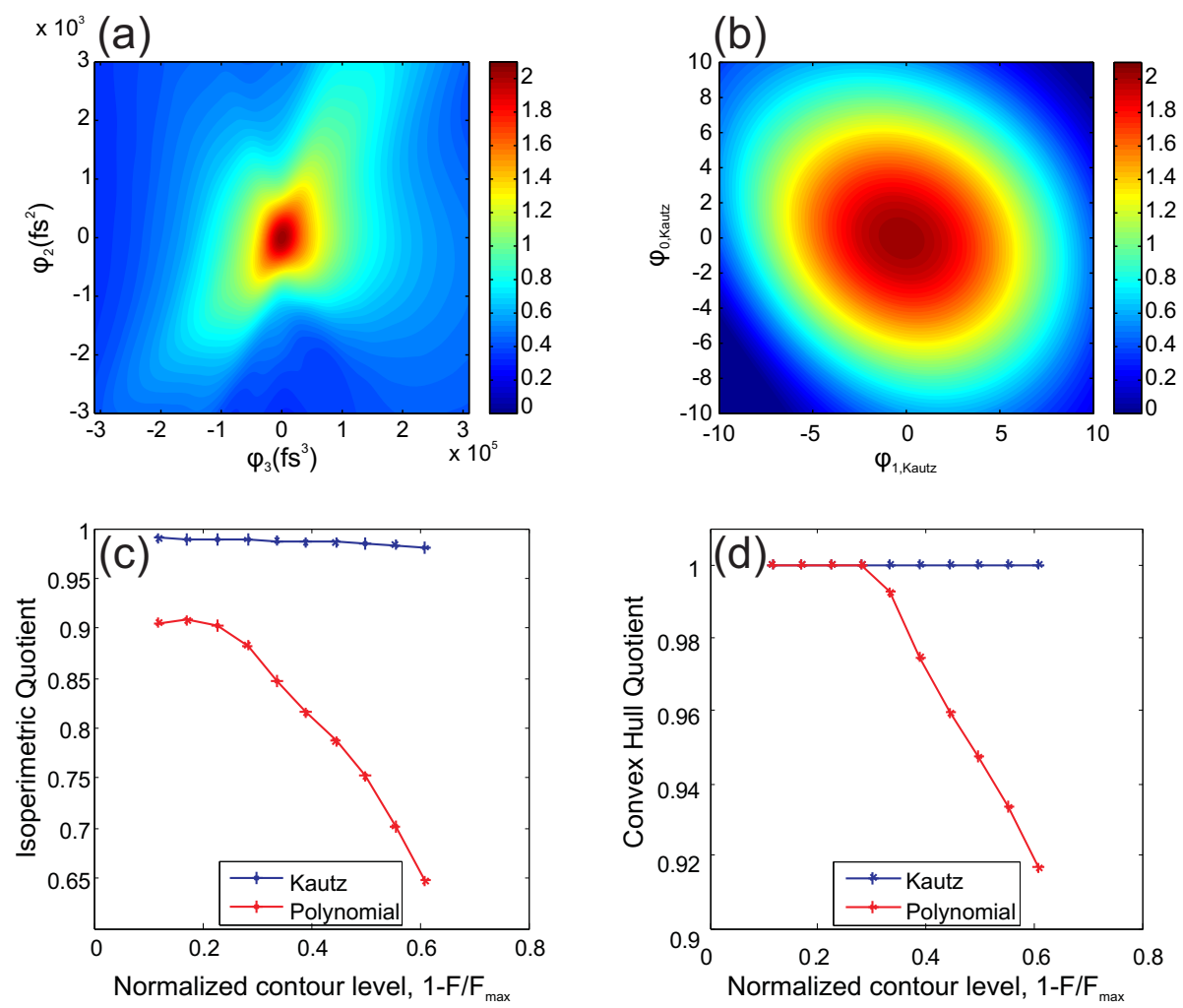

Figure 4.17: Two-dimensional landscapes of the CARS process with (a) polynomials and (b) Kautz functions, and (c) isoperimetric quotient and (d) convex hull quotient values, associated with the contours in both landscapes.

shape (lower IPQ), as the fitness drops (compared to the contours in the Kautz landscape). A higher value of IPQ for a given contour level implies more insensitivity to the direction approaching the contour.

\section{D Landscapes}

The effect of the simultaneous presence of three orders of Kautz and polynomial functions in the spectral phase (around the optimal phase) on the 3D level sets is shown in figure 4.18(a) and figure 4.18(b), respectively. The level sets shown correspond to $56 \%$ of the maximum fitness. It can be seen that individual level sets associated with polynomials feature more twisted surfaces compared to those of Kautz functions, corresponding to a more complicated optimization landscape. 

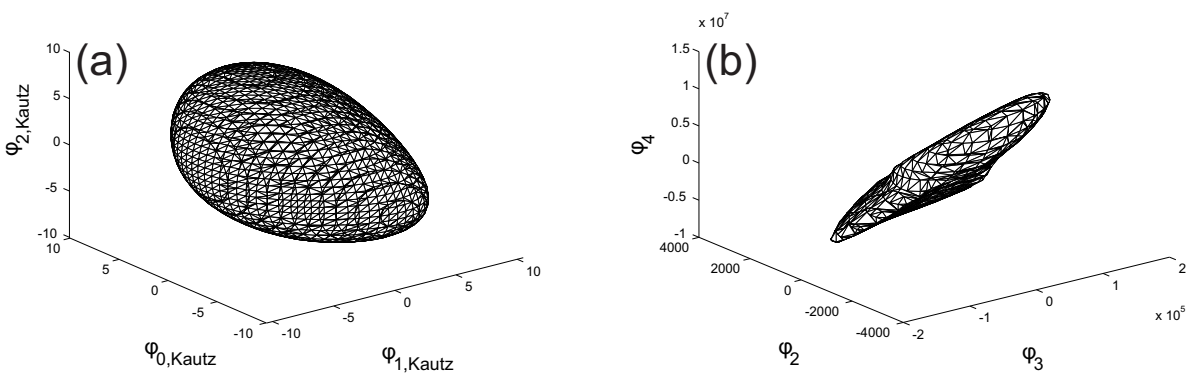

Figure 4.18: 3D level sets around the optimal point corresponding to similar fitness values (56\% of the maximum fitness) with (a) Kautz and (b) polynomial functions.

\subsubsection{Modified basis sets}

Replacing the spectral phase basis of a coherent control experiment with appropriate linear combinations of the basis functions can result in a new landscape with more convex contours, less shear, and even separable phase terms [68, 71]. This technique can also be used successfully in the case of a CARS experiment. Figure 4.19 shows two 2D landscapes (from figure 4.14) and their modified forms, when the third- and the fourth-order polynomials are modified by adding a weighted secondorder polynomial to them as shown in equations 4.13 and 4.14. The maximum values of individual polynomial phase terms over $1021 \mathrm{~cm}^{-1}$, as shown in figure 4.19 , are $\phi_{2, \max }=5.3 * 10^{2} \mathrm{fs}^{2}, \phi_{3, \max }=4.2 * 10^{4} \mathrm{fs}^{3}$, $\phi_{4, \max }=1.4 * 10^{6} \mathrm{fs}{ }^{4}$. The phase functions corresponding to these maximum values are shown in figure 4.20.

$$
\begin{array}{r}
\phi_{\text {new }}^{(4)}(\omega)=\phi^{(4)}(\omega)+\left[-0.91 \omega_{F W H M}^{2}\right] \phi^{(2)}(\omega) \\
\phi_{\text {new }}^{(3)}(\omega)=\phi^{(3)}(\omega)+\left[0.17 \omega_{F W H M}\right] \phi^{(2)}(\omega)
\end{array}
$$

with $\phi^{(2)}(\omega)$ defined as in section 4.6.3 and $\omega_{F W H M}$ denoting the spectral bandwidth. The scaling factor of $\omega_{F W H M}^{\Delta n}$, where $\Delta n$ is the difference in order between the polynomial that is added and the polynomial that is being modified, is added to scale the different polynomial orders with respect to each other. 


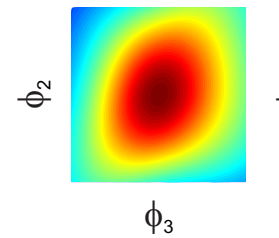

(a)

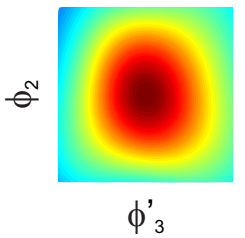

(b)

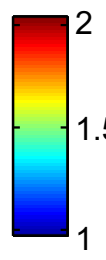

$1.5 \stackrel{2}{2}$

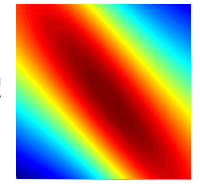

$\phi_{4}$

(c)

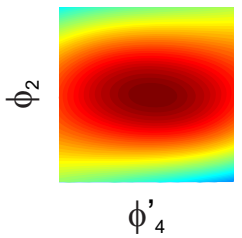

(d)

Figure 4.19: Modified 2D landscapes obtained by adding a scaled second order polynomial to (left) the third order and (right) the fourth order polynomials. $(a, c)$ show the original landscapes and $(b, d)$ show the modified landscapes.
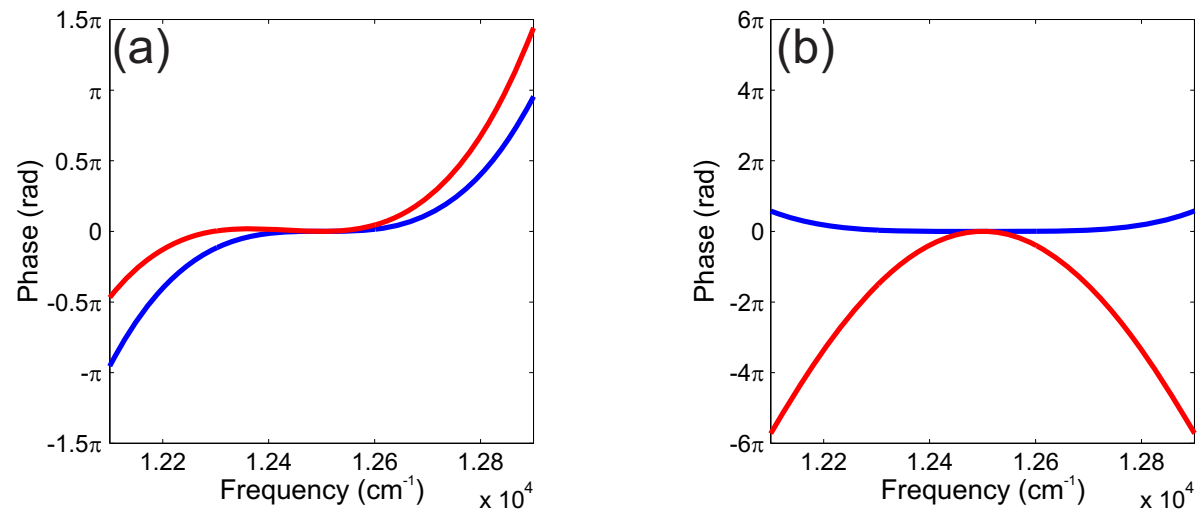

Figure 4.20: Original (blue) and modified (red) polynomial functions. (a) $\phi^{(3)}$ (blue) and $\phi_{\text {new }}^{(3)}$ (red) (b) $\phi^{(4)}$ (blue) and $\phi_{\text {new }}^{(4)}$ (red). 
Starting from one of such modified contour lines in the vicinity of the maximum point, one can find the projections of the maximum point along each phase coordinate independently. This representation reduces the required number of measurements for finding the optimal phase in the vicinity of the optimal point from $N^{2}$ to $2 N$ measurements for a given $N$ points/coordinate resolution.

In each case, the weight factor used in the modified polynomial $(-0.91$ or 0.17$)$ can be considered as the tangent of the angle between the orientations of the original and modified contour lines. However, note that this transform is a shear and not a rotation of contour lines. This is why the apparent ellipticity of the contour lines has changed [68]. 


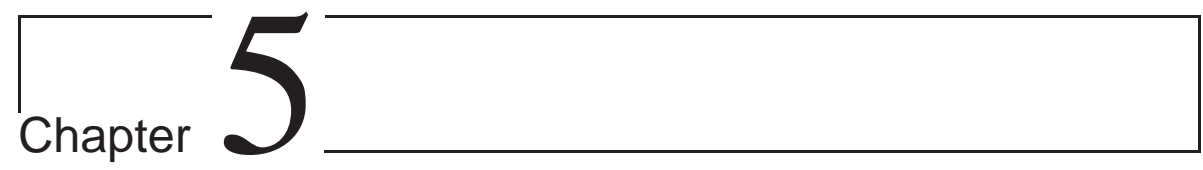

\section{Phase shaped CARS spectroscopy and imaging}

In this chapter the experimental results of phase shaped CARS are presented. This chapter covers spectroscopy and microscopy using intuitive phase shaping strategies, based on compensating the intrinsic phase profile of vibrational resonances, as well as more complex phase profiles. These complex phase profiles are obtained by applying a learning loop approach in the experimental setup and optimizing on objectives that contain multiple goals. 


\subsection{Introduction}

In conventional narrowband CARS, chemically selective imaging of a single compound in a mixture of compounds is challenging if the resonances of the different compounds overlap significantly, since the contrast is based on a single vibrational frequency. In this chapter, our broadband CARS method is demonstrated, where the contrast between different substances is based on all the resonances that are covered with our combination of input pulses, providing improved selectivity. Furthermore, the contrast is obtained from the spectrally integrated CARS intensity, facilitating easier detection.

In our approach the spectral phase of the input pulses is modified to influence the CARS response, which can be considered as a type of coherent control [72]. In coherent control, amplitude and/or phase shaping is used to influence the quantum interferences between multiple pathways, providing control over molecular processes [73, 74, 75, 76, 77]. The control of a system by using predetermined pulse shapes is known as open loop control and has been used in CARS for selective excitation of vibrational modes [31, 51, 78, 79, 80], non-resonant background suppression [32, 81], and improved detection of weak resonances [82]. In this chapter, CARS measurements based on the $\pi$ and $2 \pi$ shaping strategies presented in section 2.5 are shown. Using open loop control, the retrieval of spectral information from bulk polystyrene is shown, using both $\pi$ and $2 \pi$ phase steps. Furthermore, selective imaging in a sample of PMMA and polystyrene microspheres, using $\pi$ phase steps, is shown.

A common problem in coherent control is that solutions predicted from (optimal control) theory often do not produce good results under experimental conditions and open loop control is therefore often not optimal. In order to circumvent this problem, a closed learning loop approach is often used. In this approach, also known as adaptive feedback control [83], an algorithm is employed that measures the response of the system to evaluate and refine successive pulse shapes, until optimal control is obtained. Selective excitation of vibrational levels by using closed loop control in various CARS implementations has been reported $[48,49,84,85]$.

Chapter 4 introduced shaping strategies based on more complex phase shapes, where these complex phase shapes are obtained numer- 
ically using a learning loop approach. In this chapter a similar closed loop experimental approach is demonstrated. The phase profiles are optimized by implementing an evolutionary algorithm in the experimental setup. The optimization and background free imaging of single compounds is demonstrated using this method. Using the same approach, chemically selective imaging is shown in samples containing up to five resonant compounds. Furthermore, a comparison is made between the effectiveness of open loop control using the predicted phase shapes from theory (section 4.4) and closed loop control.

\subsection{Setup}

The broadband CARS setup uses two oscillators: a Ti:Sapphire oscillator that generates the (degenerate) pump and probe pulses, and an $\mathrm{Nd}: \mathrm{YVO}_{4}$ oscillator that is used for the Stokes pulse. These two oscillators are synchronized using an electronic and optical feedback system. Further details about the oscillators and the synchronization setup can be found in section 3.2. A schematic overview of the broadband CARS setup used to obtain the measurements in this chapter is shown in figure 5.1 .

The pump light that is not being used for the synchronization $( \pm 200$ $\mathrm{mW}$ ) is sent to the phase shaping setup. Two different phase shaping setups have been used for these experiments. Both are based on a reflective 4-f zero dispersion layout [86]. The incident light is dispersed by a ruled diffraction grating, after which the different wavelengths of the pulse propagate at different angles. The different wavelengths are subsequently focused by a cylindrical mirror, which is positioned at one focal length from the grating. This cylindrical mirror causes the wavelengths that are propagating at different angles to be focused at different spatial positions. The spatial light modulator (SLM) is placed in the focus of the cylindrical mirror, so that different pixels of the SLM coincide with the focus of different wavelength ranges, allowing wavelength specific phase modulation by the SLM. The pulse is reflected off the backside of the SLM and propagates back through the cylindrical mirror and the grating, which spatially recombines the different wavelengths into a single pulse. A slight vertical tilt $\left(1 \mathrm{~cm}\right.$ over $\left.2.73 \mathrm{~m}, 0.21^{\circ}\right)$ is used to separate the outgoing beam from the incoming beam. 


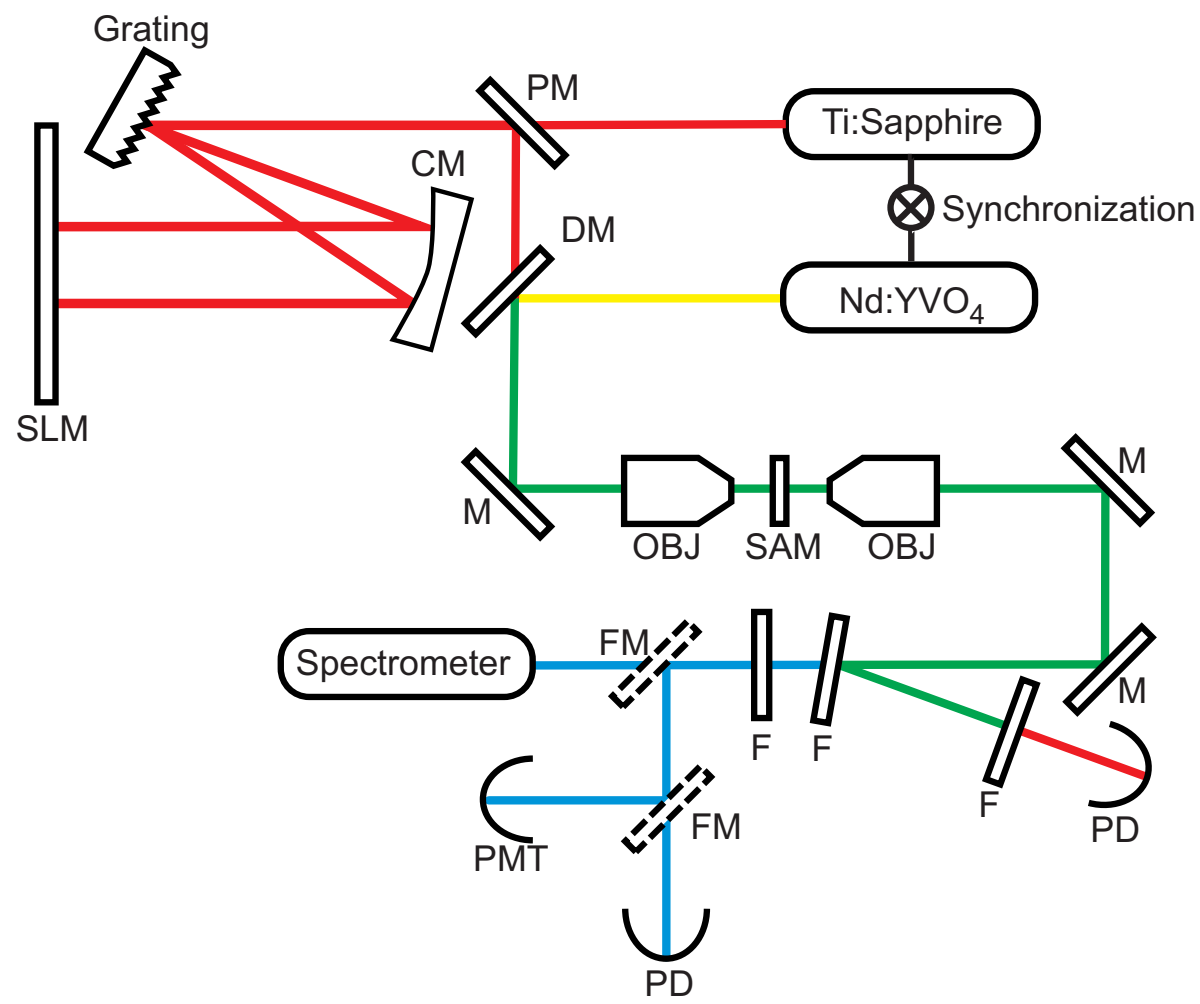

Figure 5.1: Schematic overview of the broadband CARS setup. $C M=$ Cylindrical Mirror, SLM = Spatial Light Modulator, PM = Pickoff Mirror, $D M=$ Dichroic Mirror, $M=$ Mirror, OBJ = Microscope Objective, $S A M=$ Sample, $P D=$ Photodiode, $F=$ Filter, $F M=$ Flip Mirror, $P M T$ $=$ Photomultiplier Tube. 
For the measurements with the $\pi$ and $2 \pi$ phase steps (section 5.4), a 4096 pixel liquid crystal phase-only modulator (Boulder Nonlinear Systems), with a pixel pitch of $1.8 \mu \mathrm{m}$, is used in combination with a 830 lines/mm grating and a cylindrical mirror with focal length $\mathrm{f}=50 \mathrm{~mm}$. This corresponds to a frequency width of $12 \mathrm{GHz} /$ pixel $( \pm 0.03 \mathrm{~nm})$. In this setup, six pixels share spectral information, so that the effective frequency resolution is $72 \mathrm{GHz}$. A 0.65 numerical aperture (NA) goldcoated reflective air objective (Newport $74 \mathrm{x}$ ) was used as the excitation objective and a $0.5 \mathrm{NA}$ extra long working distance air objective (Nikon M-plan 40x) was used as a collection objective.

For the complex phase profile measurements (section 5.5) a 640 pixel phase and amplitude liquid crystal modulator (CRi Inc.), with a pixel pitch of $100 \mu \mathrm{m}$ is used, which is operated in a phase shaping only mode. In this setup, a grating of 830 lines/mm is used in combination with a cylindrical mirror with a focal length of $\mathrm{f}=597 \mathrm{~mm}$. This corresponds to a frequency width of $94 \mathrm{GHz} /$ pixel $( \pm 0.2 \mathrm{~nm})$. A $0.6 \mathrm{NA}$ near infrared air objective (Nachet Plan Fluor 40x) was used as excitation objective and a $0.75 \mathrm{NA}$ air objective (Zeiss Plan-Neofluar 60x) was used as a collection objective.

The objectives are mounted on a 3-dimensional translation stage (CVI Melles Griot) to carefully overlap their focal volumes. After the collection objective, the CARS signal passes through a long-pass filter (Thorlabs FM01) to filter out the excitation light. This excitation light is collected by a photodiode with a bandpass filter (Thorlabs FB800-40) in front and is used to detect the optical transmission of the pump beam through the sample.

Detection of the CARS signal was either done spectrally resolved, using a 3648-pixel silicon based spectrometer (Avantes AvaSpec-3648USB2), or spectrally unresolved, with a photomultiplier tube (Hamamatsu) or a silicon photodiode. To filter the CARS signal from any remaining excitation light, a filterset containing multiple filters (Thorlabs FM01, 2x Chroma 655-50-2P, 2x Thorlabs FB650-40) was used.

For the microscopy measurements a 2-dimensional piezo-scanner (Piezosystem Jena) was used to scan the sample through the focus. The piezo-scanner has a scan range of approximately 90x90 micrometer. Also, a lock-in amplifier and chopper were used to improve the signal to noise ratio. The chopper modulates the Stokes beam and the 
resulting modulation on the CARS signal was detected using a lock-in amplifier (EG\&G Princeton Applied Research Model 5210) . Modulating the Stokes beam has the advantage that the photomultiplier tube is insensitive to the wavelength of the Stokes pulse, so that any Stokes light falling onto the detector is not picked up by the lock-in amplifier.

\subsection{Sample materials}

The CARS measurements are performed on a variety of plastics and solvents. The bulk polystyrene is obtained by cutting out a plastic piece from a translucent CD-case.

The $4 \mu \mathrm{m}$ and $20 \mu \mathrm{m}$ PMMA and polystyrene beads and the $12 \mu \mathrm{m}$ melamine resin microbeads are monodisperse microbead solutions obtained from Sigma-Aldrich. They are diluted using demineralized water.

The polyethene beads are obtained from Cospheric Microspheres and are specified to have $95 \%$ of the microbeads within a size of $20-27 \mu \mathrm{m}$. They have been suspended in ethanol.

The acetone and ethanol is obtained from Sigma-Aldrich and has a specified purity of $\geq 99 \%$. The liquid paraffin is obtained from J.T.Baker.

The microbead samples were prepared on glass coverslides of $170 \mu \mathrm{m}$ thickness. The end of the thin coverslide is glued onto a microscope slide of $1 \mathrm{~mm}$ thickness to facilitate mounting in the sample scanner.

\subsection{Phase step profiles}

In this section, CARS spectroscopy and microscopy measurements are presented, using both $\pi$ and $2 \pi$ shaping strategies. The retrieval of spectroscopic information from a bulk sample of polystyrene is shown as well as the ability to do chemically selective imaging in a sample consisting of polystyrene and PMMA microspheres.

Our shaping strategies assume a flat phase on the pump and probe pulse before any phase shapes are applied. Any residual phase that is caused by dispersion of, for example, lenses or other optics is therefore not taken into account. Before applying any phase profiles, this residual 
phase is compensated by optimizing the second harmonic generation signal generated by the pump and probe pulse in a $\mathrm{BiB}_{3} \mathrm{O}_{6}$ (BIBO) crystal. The second harmonic generation signal is maximized by optimization of the excitation phase, using a derandomized evolution strategy (DR2) $[65,87]$ to optimize the coefficients of a polynomial basis set up to 15 th order. We apply our phase step profile on top of the phase profile that compensates for the residual dispersion.

\subsection{1 $\pi$ phase step}

\section{Spectroscopy}

As shown in section 2.5, a scanning $\pi$ phase step can be used to obtain spectroscopic information. Here we apply this sweeping $\pi$ phase step to a bulk sample of polystyrene (PS). A $\pi$ phase step is scanned through the Ti:Sapphire (pump and probe) spectrum and for every position of the phase step, the CARS spectrum is measured.

We measure the CARS spectra for a positively sloped phase step (figure 5.2(b)), a negatively sloped phase step figure 5.2(d), and for a Fourier-limited pulse. Spectra are integrated for 300 milliseconds and a complete 2D spectrum is measured in approximately five minutes. The 2D difference spectrum for PS, obtained by subtracting the negative step measurements from the positive step measurement, can be found in figure 5.2(f). A phase step corresponding to a resonance with a linewidth of $10 \mathrm{~cm}^{-1}(0.6 \mathrm{~nm})$ was scanned over 251 points with a step size of $0.1793 \mathrm{THz}\left(4.65 \mathrm{~cm}^{-1}\right)$. The width was chosen to match the vibrational transitions in this spectral region (on average). In figure 5.2, the theoretically calculated $2 \mathrm{D}$ difference spectra are also shown for comparison. For the simulated spectra a model of the vibrational response of polystyrene that uses three resonances was used (see figure 4.10), with a constant non-resonant background of $15 \%$ of the imaginary part of the main peak of polystyrene. The pump pulse is defined as a Gaussian pulse with a center wavelength of $809.2 \mathrm{~nm}\left(370.2 \mathrm{THz}, 12348.5 \mathrm{~cm}^{-1}\right)$ and an intensity FWHM bandwidth of $16.41 \mathrm{~nm}\left(7.5 \mathrm{THz}, 250 \mathrm{~cm}^{-1}\right)$ and the Stokes pulse is assumed to be infinitely narrow with a center frequency of $9395.85 \mathrm{~cm}^{-1}(1064.3 \mathrm{~nm})$. The intensity in the simulations was scaled to get a good fit between the theoretical 2D difference spectrum and the experimental $2 \mathrm{D}$ difference spectrum. These parameters are also used for the calculations shown in figure 5.3. 

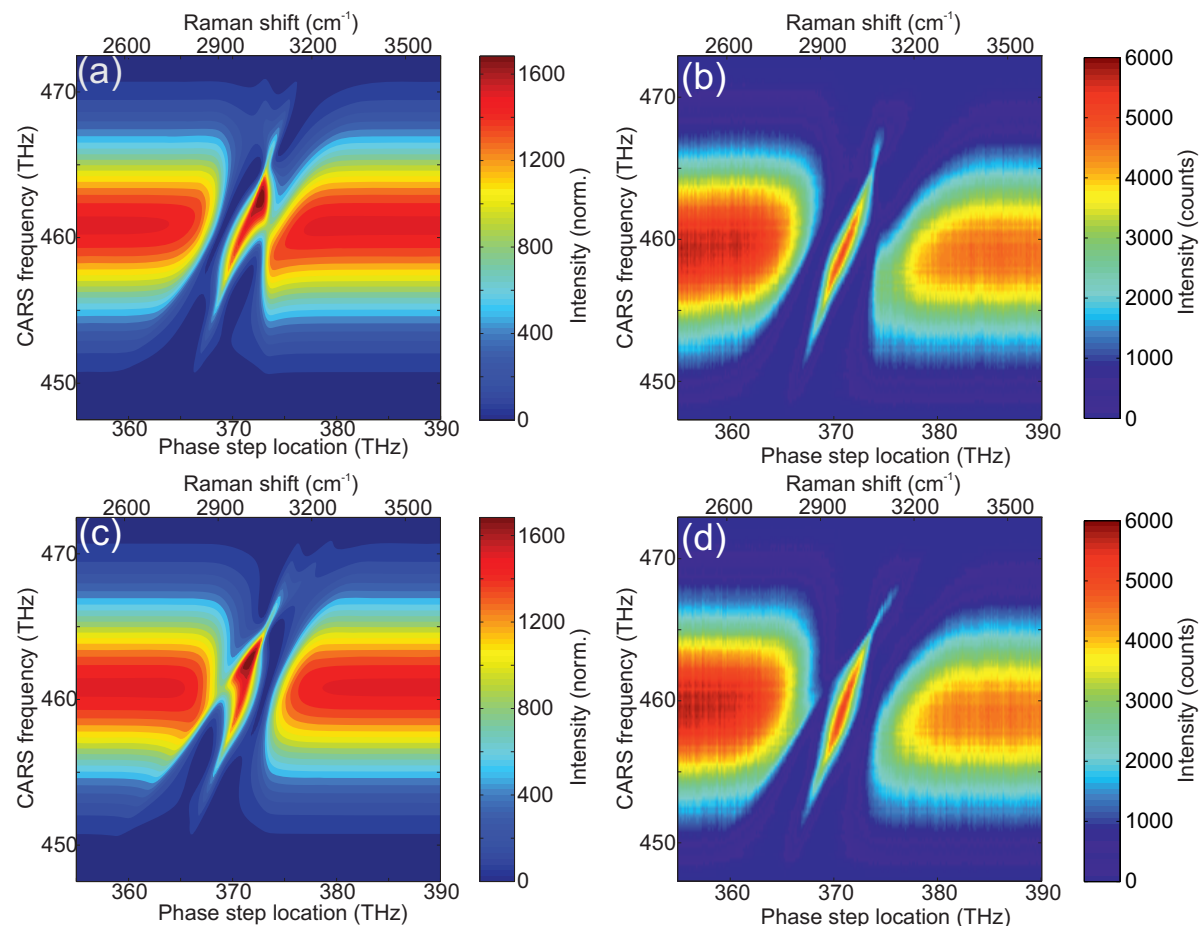

Raman shift $\left(\mathrm{cm}^{-1}\right)$
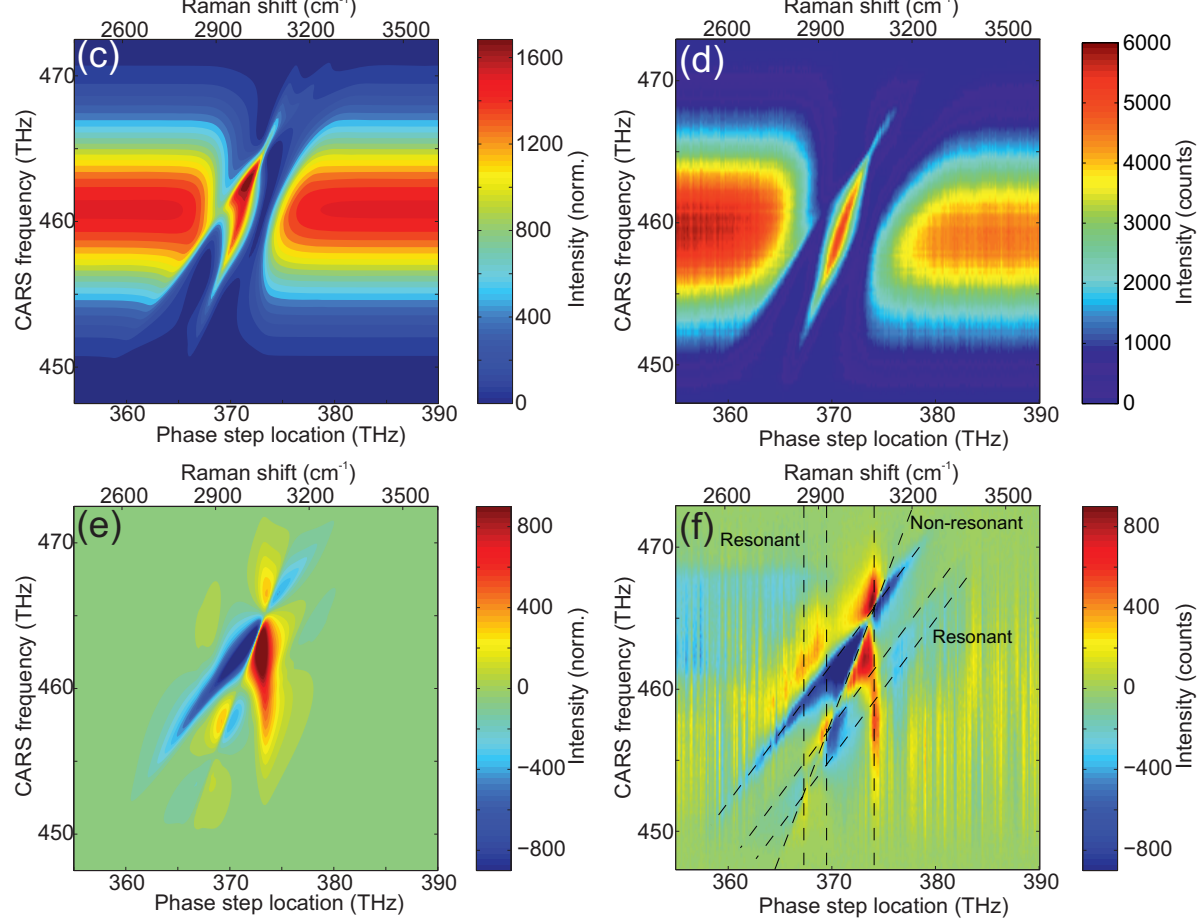

Figure 5.2: Theoretical $(a, c, e)$ and measured $(b, d, f) 2 D$ spectra for bulk polystyrene. The difference spectrum $(e, f)$ is obtained by subtracting the spectrum for a negative phase step $(c, d)$ from the spectrum obtained with a positive phase step $(a, b)$. The CARS (difference) spectrum (vertical) is shown as a function of the location of the phase step in the pump and probe spectrum (horizontal). 
In figure $5.2(\mathrm{f})$, both the sloped and vertical contributions (see section 2.5.3) from the vibrational resonances of PS are indicated with dashed lines. The contribution from the Raman vibration at $3059 \mathrm{~cm}^{-1}$ is visible at $373.5 \mathrm{THz}\left(3062 \mathrm{~cm}^{-1}\right)$. A second, weaker resonance is also revealed. The vertical lines indicate the crossing point of the resonant line features of both resonances with the non-resonant line. The second, weaker resonance is located at $369.6 \mathrm{THz}\left(\right.$ or $\left.2930 \mathrm{~cm}^{-1}\right)$. This feature can be assigned to a Raman resonance of PS at $2910 \mathrm{~cm}^{-1}$. Furthermore, there is a signal evident from an even weaker resonance at 367.5 $\mathrm{THz}$ (or $2862 \mathrm{~cm}^{-1}$ ). This signal is assigned to the weak resonance at $2857 \mathrm{~cm}^{-1}[88]$. The difference in intensity between the simulations and the measurements for the positive and negative step spectra (figure $5.2(\mathrm{a}-\mathrm{d}))$ is most likely due to a difference in non-resonant background.

\section{Imaging}

A sample consisting of a mixture of $4 \mu \mathrm{m}$ PS and $4 \mu \mathrm{m}$ PMMA microspheres dried on a glass surface is used for imaging. Selective imaging is demonstrated by applying a positive and negative $\pi$ phase step near the main resonance for PMMA or PS. A suitable phase step location can be chosen by looking at the integrated CARS difference signal from a $\pi$ phase step sweep, as shown in figure 5.3. The integrated CARS difference signal can be obtained by spectrally integrating the $2 \mathrm{D}$ difference spectrum. A phase step at $370.2 \mathrm{THz}$ is chosen for selective imaging of PMMA and a phase step at $373.2 \mathrm{THz}$ is chosen for selective imaging of PS, as shown in figure 5.3(a).

Measurements are taken with a $10 \mathrm{~ms} /$ pixel integration time. The CARS signal is detected using a photomultiplier tube and a lock-in amplifier. The integration time on the lock-in amplifier is chosen to be $3 \mathrm{~ms}$, with a $1.71 \mathrm{kHz}$ modulation frequency. The phase patterns are switched between positive and negative per line as the liquid crystals in the LCD require roughly $200 \mathrm{~ms}$ to completely stabilize. Each line is measured three times (positive step, negative step, Fourier limited) so that the total image takes about $30 \mathrm{~min}$ to acquire.

Measurements with positive and negative $\pi$ phase steps at $370.2 \mathrm{THz}$ are shown in figure 5.4(a) and 5.4(b), respectively. For an image with a Fourier-limited pulse, shown in figure 5.4(c), the difference in intensity between the PMMA and PS beads is due primarily to the larger scat- 

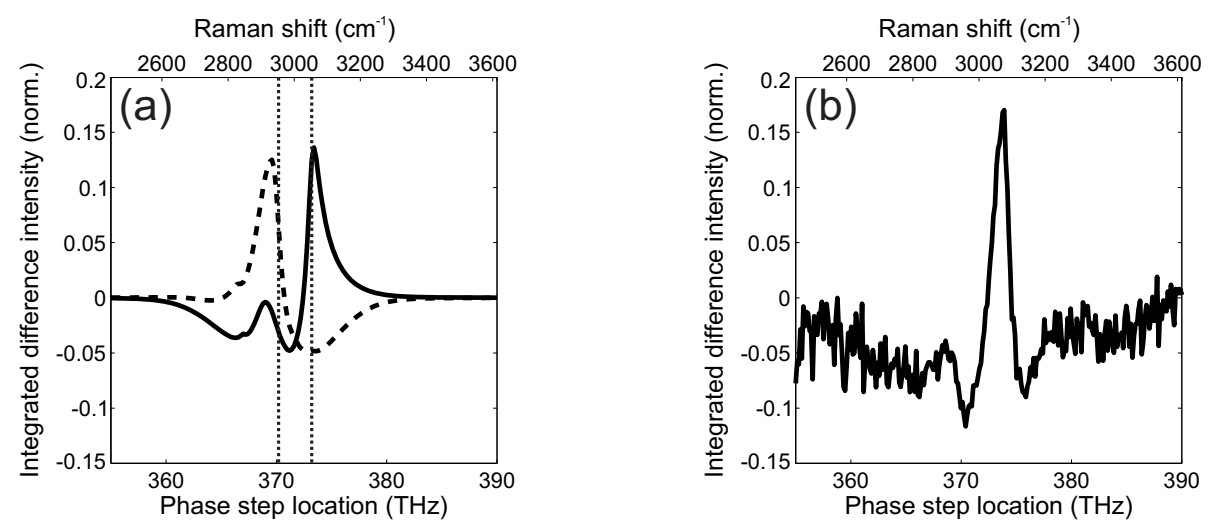

Figure 5.3: (a) Calculated CARS difference intensity as a function of phase step location for PS (solid) and PMMA (dashed). The dotted vertical lines indicate the two phase step locations that were chosen for imaging. (b) Experimentally measured difference intensity as a function of phase step location for PS. The intensities in $(a, b)$ are normalized to the CARS intensity obtained with an unshaped (Fourier-limited) pulse.

tering cross section of PS compared to PMMA. For the positive phase step at $370.2 \mathrm{THz}$ an increase in intensity of the PMMA beads relative to the PS beads is observed, when compared to the Fourier-limited case. For the negative phase step the relative intensity of the PMMA beads is observed to be roughly the same as in the Fourier-limited case.

The intensity difference (between signals obtained with positive and negative phase steps) for the PMMA and PS beads varies greatly from positive to negative as a function of the step position (see figure 5.3). By switching the location of the phase step, the amount of obtained difference signal from PS and PMMA can be modified. The difference intensity images for the $\pi$ phase step centered near the main resonance of PMMA (370.2 THz) and near the main resonance of PS (373.2 THz) are presented in figure 5.5. These difference images are obtained by subtracting the negative step image from the positive step image.

In the difference intensity image for the phase step positioned near the main resonance of PMMA (figure 5.5(a)) the PMMA beads are visible, while the PS beads are suppressed. For the phase step positioned near the main resonance of PS (figure 5.5(b)) the PS beads are visible, while the PMMA beads are suppressed. The PMMA beads are still somewhat visible, but with a negative (difference) intensity as opposed 
(a)

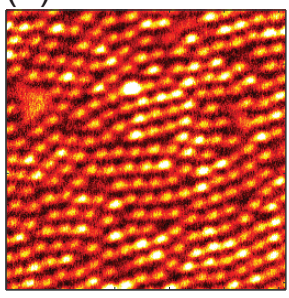

(b)

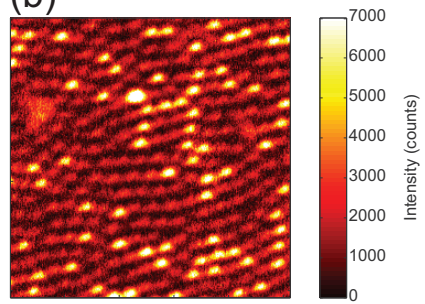

(c)

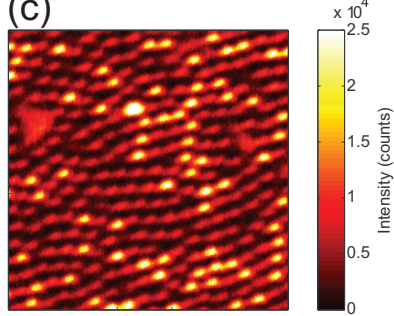

Figure 5.4: Shaped CARS imaging in a sample of polystyrene and PMMA microbeads. (a) Image taken with a positive phase step at 370.2 THz. (b) Image taken with a negative phase step at 370.2 THz. (c) Image taken with a Fourier-limited pulse. Image size is $256 x 256$ pixels (approximately 60x60 $\mu \mathrm{m}$ ).

(a)

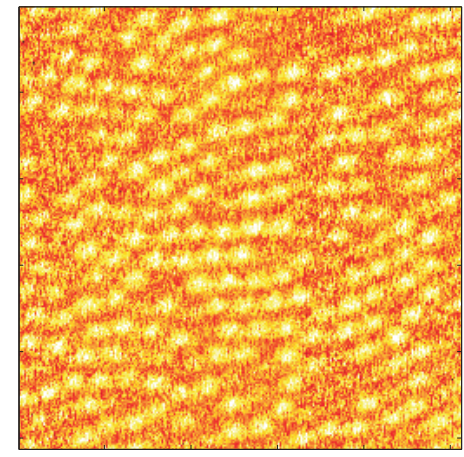

(b)

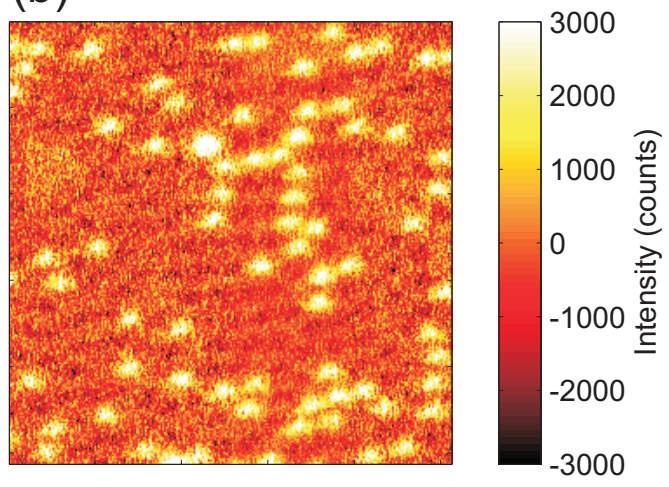

Figure 5.5: Difference CARS intensity images in a sample of polystyrene and PMMA microbeads with a phase step at $370.2 \mathrm{THz}($ a) and 373.2 $\mathrm{THz}(\mathrm{b})$. Image size is $256 x 256$ pixels (approximately $60 x 60 \mu \mathrm{m}$ ). 

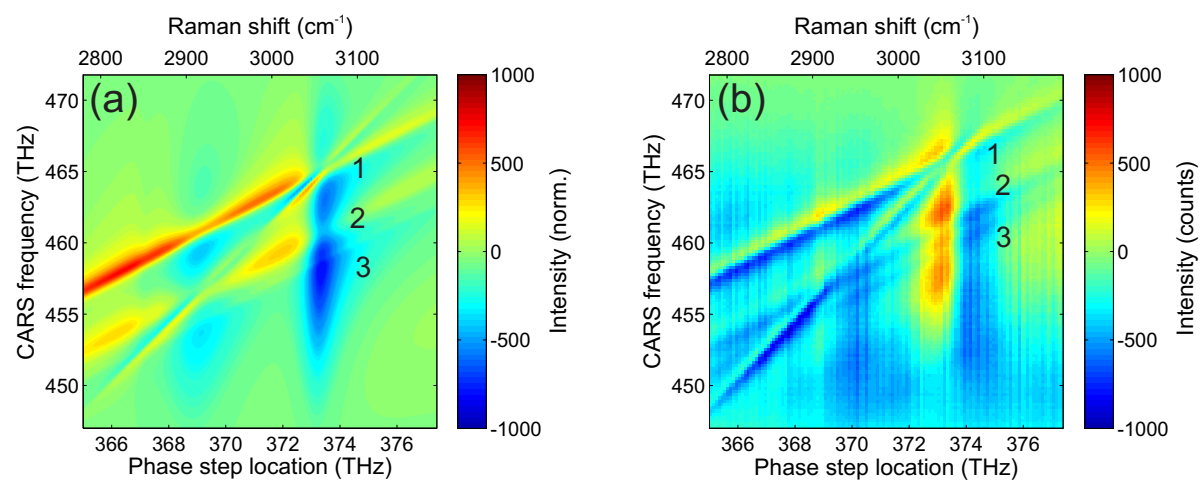

Figure 5.6: $2 \pi$ phase step scan on polystyrene. (a) Theoretical $2 D$ difference spectrum of bulk polystyrene. (b) Measured $2 D$ difference spectrum. The numbers indicate the features caused by the three main resonances of polystyrene.

to the positive intensity of the PS beads.

\subsection{2 $2 \pi$ phase step}

Section 2.5.4 explores the possibilities and advantages of sweeping a $2 \pi$ phase step through the pump and probe spectrum and recording the CARS spectrum for each phase step location. This procedure is applied to both a positively sloped phase step and a negatively sloped phase step, corresponding to a resonance with a linewidth of $10 \mathrm{~cm}^{-1}(0.6 \mathrm{~nm})$. The residual dispersion on the pump pulse is corrected in the same way as described in section 5.4 (page 86). The results of such a measurement on bulk polystyrene are shown in figure 5.6. For the simulated difference spectrum a model that uses three resonances to model the vibrational response of polystyrene was used (see figure 4.10), with a constant nonresonant background of $10 \%$ of the imaginary part of the main peak of polystyrene. The pump pulse is defined as a Gaussian pulse with a center wavelength of $813.5 \mathrm{~nm}\left(368.5 \mathrm{THz}, 12291.8 \mathrm{~cm}^{-1}\right)$ and an intensity FWHM bandwidth of $27.5 \mathrm{~nm}\left(12.5 \mathrm{THz}, 417 \mathrm{~cm}^{-1}\right)$. The Stokes pulse is assumed to be infinitely narrow with a center frequency of 9395.85 $\mathrm{cm}^{-1}(1064.3 \mathrm{~nm})$. The intensity in the simulations was scaled to get a good fit between the theoretical 2D difference spectrum and the experimental 2D difference spectrum.

It can be seen from figure 5.6 that the measured 2D spectrum (figure 
5.6(b)) matches qualitatively with the theoretically predicted spectrum (figure 5.6(a)). The numbers in figure 5.6 indicate the features caused by the three main resonances of polystyrene. The predominantly negative difference intensity in the experimental measurement is caused by errors introduced by the SLM. Applying a $2 \pi$ phase step on the SLM uses a large part of the modulation range $(3.5 \pi)$, so that there is little room for residual phase compensation, without introducing additional phase jumps. Furthermore, this phase wrapping occurs at different positions for the positive step and the negative step, so that measurements with different artifacts are subtracted from each other, leading to imperfections in the difference spectrum. Errors in the calibration of the SLM will also lead to imperfections in the subtraction.

\subsection{Complex profiles}

As discussed in chapter 4, complex phase profiles show promise for chemically selective imaging in samples with multiple resonant compounds. In this section, chemically selective imaging is demonstrated using complex phase profiles that are obtained using a learning loop approach. Chemically selective imaging is demonstrated in samples with a single resonant compound as well as in samples with multiple resonant compounds.

To reduce the residual phase on the pump pulse, an optimization of the pump pulse phase is performed, using the non-resonant CARS signal from water as a feedback signal. Because this non-resonant signal has a flat phase response, the CARS signal it generates is maximized for a transform limited pulse (flat spectral phase). The optimization is performed using Legendre polynomials, which are normalized between $-2 \Delta \omega$ and $2 \Delta \omega$, where $\Delta \omega$ is the half-width at half maximum (HWHM) bandwidth of the pump pulse. These Legendre polynomials form an orthogonal basis set and show good performance for laser pulse compression [71]. The phase profile that is applied is described using the first eight Legendre polynomials $\left(L_{0}-L_{7}\right)$. The first Legendre polynomial $\left(L_{0}\right)$ contains only constant phase and the second Legendre polynomial $\left(L_{1}\right)$ contains only linear phase terms. Because our CARS implementation is insensitive to constant and linear phase offsets on the pump pulse, we omit these terms from the optimization, leaving six Legendre polynomials $\left(L_{2}-L_{7}\right)$ with their corresponding scaling factors, as shown 
in equation 5.1.

$$
\phi(\omega)=\sum_{k=2}^{7} \alpha_{k} L_{k}
$$

The coefficients $\alpha_{k}$ are optimized using CMA-ES, using 100 generations, with 5 parents and a population size of 10 per generation. It was found that the optimized phase provided no significant increase $(<1 \%)$ in non-resonant CARS signal nor in the CARS signal from a polystyrene microsphere. Therefore, the residual phase is assumed to be small, which can also be seen in figure 5.7.

Figure 5.7(a) shows the phase profile that results from the nonresonant CARS optimization. Figure 5.7(b) shows the phase profile from figure 5.7(a), but with a linear fit (fitted from $790 \mathrm{~nm}$ to $820 \mathrm{~nm}$ ) subtracted from the phase, to remove any constant and linear phase contributions over this wavelength range. From 5.7(b) it can be seen that the residual phase on the pump and probe pulse is small and is mostly concentrated in the wings. Because there is no increase in signal for the compensated pulse compared to an unshaped pulse, it can be concluded that the phase wrapping introduced by compensating the residual phase (as described in section 5.4) introduces additional errors which offset the gain from compensating the residual phase. Since there is no gain in signal and we wanted to avoid this phase wrapping, we chose to avoid residual phase compensation and apply the phase profiles in our experiments directly on our unshaped pulse.

\subsubsection{Single compound optimization}

First we consider a sample consisting of a single resonant compound. The goal is to optimize the difference CARS signal from this single compound. The sample consists of $20 \mu \mathrm{m}$ diameter poly(methyl methacrylate) (PMMA) microspheres in demineralized water, dried on a glass coverslide (170 $\mu \mathrm{m}$ thickness) and subsequently covered with a thin layer $( \pm 200 \mu \mathrm{m})$ of demineralized water and another coverslide. Even though the $\mathrm{O}-\mathrm{H}$ stretch vibration from water has a tail that extends to 3000 $\mathrm{cm}^{-1}$, the water is considered to be almost completely non-resonant, since the $\mathrm{O}-\mathrm{H}$ resonance is very broad and the amplitude at the $\mathrm{C}-\mathrm{H}$ region is small [89]. 

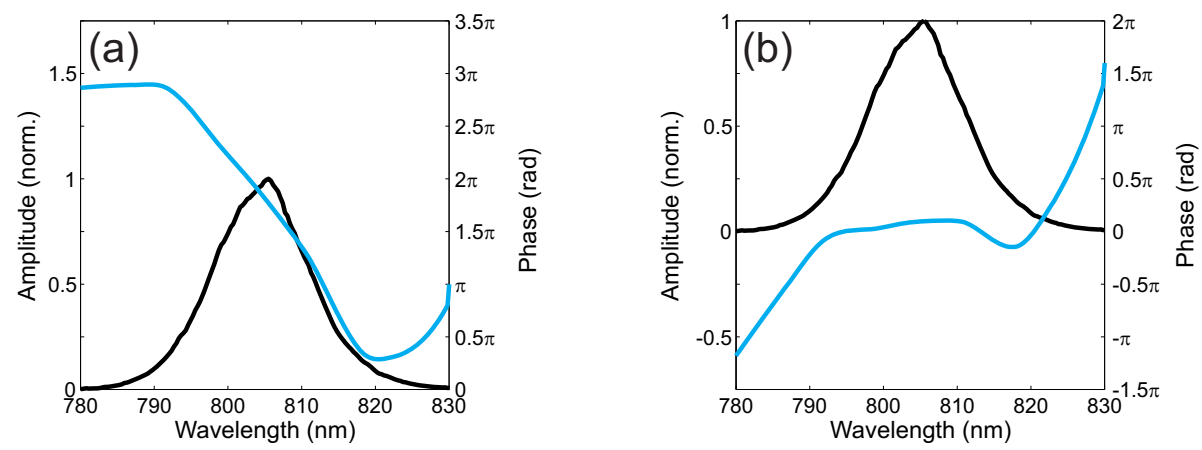

Figure 5.7: (a) Pump and probe intensity (black) and phase (cyan) resulting from the non-resonant CARS optimization. (b) The pump and probe intensity (black) and phase (cyan) as shown in (a), but with a linear fit (fitted from $790 \mathrm{~nm}$ to $820 \mathrm{~nm}$ ) subtracted from it to remove any constant and linear phase terms over this range.

We use CMA-ES for the optimization of the phase of the pump and probe pulse, using 100 generations with 5 parents and a population size of 10 per generation. The phase profile is optimized on 60 points spread evenly over $60 \mathrm{~nm}$. The phase profile is interpolated, using cubic spline interpolation, to obtain the phase values for the individual pixels of the SLM.

For every trial phase profile $\phi(\omega)$, the difference in CARS signal generated by a pulse with phase $\phi(\omega)$ and by a pulse with phase $-\phi(\omega)$ is measured for both the PMMA microspheres and the non-resonant background (water). The fitness is defined as the difference intensity obtained from the PMMA minus twice the difference intensity from the non-resonant background. We subtract the background in the fitness to suppress unwanted effects caused by, for example, slight errors in the calibration or crosstalk in the SLM.

After the optimal phase profile $\phi_{\text {opt }}(\omega)$ has been determined, this phase profile is used for imaging (see figure 5.8). An image is made with a pulse with phase $\phi_{\text {opt }}(\omega)$ (figure 5.8(a)) and another image with phase $-\phi_{\text {opt }}(\omega)$ (figure 5.8(b)). The difference between these images (figure $5.8(\mathrm{c})$ ) gives a non-resonant background free image with improved contrast. An image with an unshaped pulse is recorded for comparison (figure 5.8(d)). Each image is 200x200 pixels and was acquired in approximately 17 minutes. 

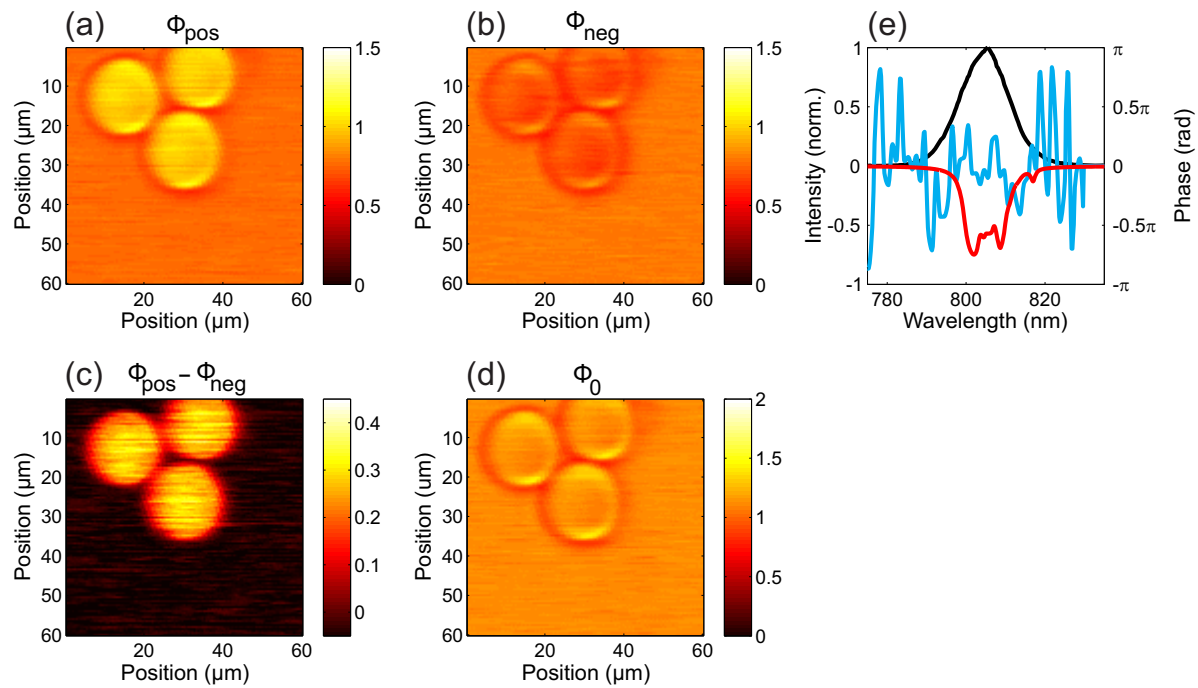

Figure 5.8: Optimized imaging of PMMA microspheres in water using the experimentally optimized phase. (a) Image taken with excitation phase $\phi_{\text {opt }}(\omega)$. (b) Image taken with excitation phase $-\phi_{\text {opt }}(\omega)$. (c) Non-resonant background free difference image obtained by subtracting (b) from (a). (d) Image taken with an unshaped excitation pulse. (e) Pump and probe spectrum (black) and experimentally optimized excitation phase for PMMA (cyan). The vibrational phase of PMMA (red), shifted by the Stokes frequency, is shown for comparison.

From figure 5.8(d) it can be seen that the contrast for an unshaped pulse is low due to the large non-resonant background. The amount of CARS signal from the PMMA microspheres is similar to that of the non-resonant background and the microspheres are visible largely due to the diffraction at the edges of the spheres. In the difference image (figure 5.8(c)) it can be seen that the non-resonant background has been suppressed and that the contrast has been strongly enhanced, making the PMMA microspheres clearly visible. The contrast ratio is now on the order of 10:1.

\section{Comparison with theory}

To compare the experimentally optimized phase profiles to the numerically optimized phase profiles, we measure CARS images using the numerically optimized phase for PMMA. Figure 5.9 shows the resulting 

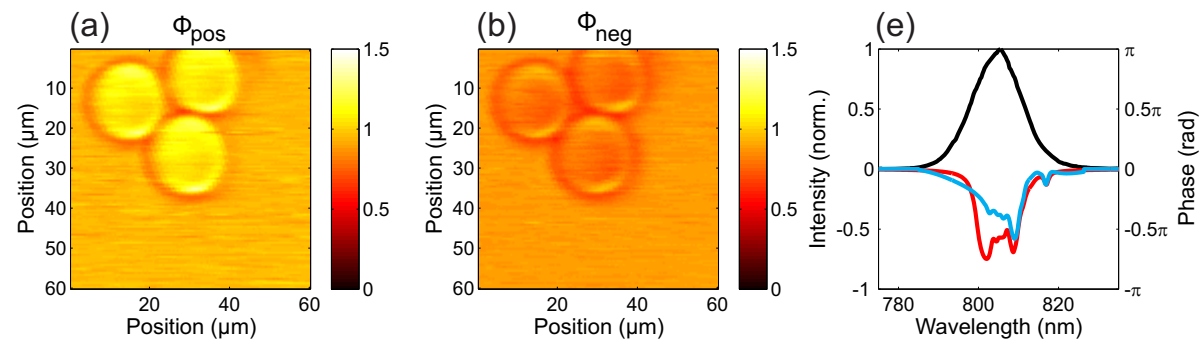

(c) $\Phi_{\text {pos }}-\Phi_{\text {neg }}$
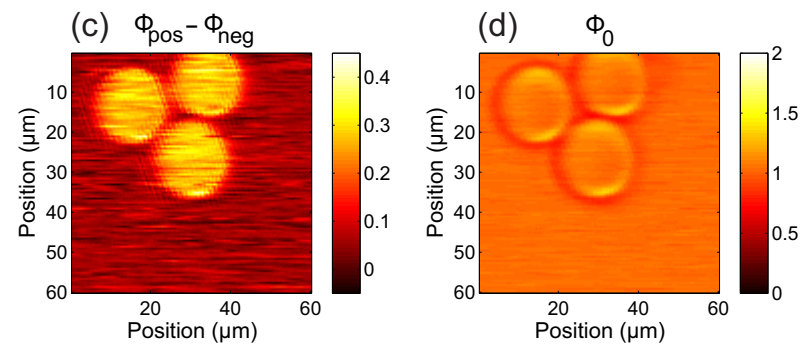

Figure 5.9: Optimized imaging of PMMA microspheres in water using the numerically optimized phase. (a) Image taken with excitation phase $\phi_{\text {num }}(\omega)$. (b) Image taken with excitation phase $-\phi_{\text {num }}(\omega)$. (c) Difference image obtained by subtracting (b) from (a). (d) Image taken with an unshaped excitation pulse. (e) Pump and probe spectrum (black) and numerically optimized excitation phase for PMMA (cyan). The vibrational phase of PMMA (red), shifted by the Stokes frequency, is shown for comparison.

images on the same sample of PMMA microspheres in water, now using the numerically optimized phase for PMMA (see section 4.4).

It can be seen from figure 5.9(c) that the contrast in the difference image is improved compared to the image taken with the unshaped pulse (figure 5.9(d)). The difference intensity from the PMMA microspheres is similar to that in figure 5.8. Using the numerically optimized phase, however, there is some remaining non-resonant background signal in the difference image. The resulting contrast is now 4:1, which is a factor 2.5 lower than the contrast obtained with the experimentally optimized phase.

We also compare the phase from the experimental optimization to theory by comparing the difference CARS intensity obtained in the experiment to the difference intensity that is predicted numerically using the pump and probe spectrum and phase from the experiment as input 


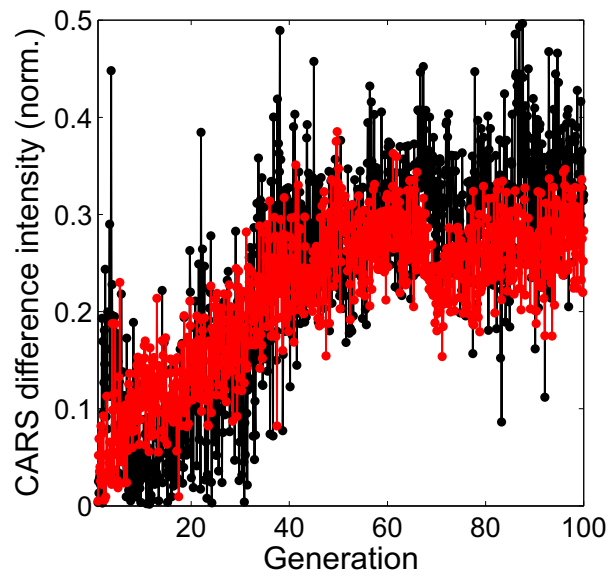

Figure 5.10: Normalized experimental (red) and theoretical (black) difference CARS intensity as a function of generation.

parameters. The results are shown in figure 5.10. Both the experimental and theoretical difference intensity are normalized to the CARS intensity obtained from pure polystyrene with a (near) transform-limited pulse. For the normalization of the theoretical fitness, a constant non-resonant background of $15 \%$ of the imaginary part of the main resonance of polystyrene was assumed.

It can be seen from figure 5.10 that the experimental and theoretical difference intensities show a similar trend. The numerically calculated intensity differs from the experimental intensity primarily at the start and the end of the optimization. These differences, as well as the remaining non-resonant background in figure 5.9, are most likely caused by calibration errors in the SLM or residual phase on the pump and probe pulse.

\subsubsection{Multiple compound mixtures}

While the optimization of the phase for a single compound is useful for high contrast, non-resonant background free imaging, the real power of these complex phase shapes lies in chemically selective imaging in samples with multiple resonant compounds. As will be demonstrated in this section, complex phase shapes can be used to enhance the signal of the compound of interest in the CARS difference images, while simultaneously suppressing the unwanted contributions of other resonant 
compounds.

As a sample, a mixture of $20 \mu \mathrm{m}$ PMMA microspheres and $20 \mu \mathrm{m}$ polystyrene (PS) microspheres in demineralized water, and 20-27 $\mu \mathrm{m}$ polyethene (PE) microspheres in ethanol, is dried on a glass coverslide. The dried beads are covered with a layer $( \pm 200 \mu \mathrm{m})$ of liquid paraffin and another coverslide on top. This means there are four compounds in the sample. Figure 5.11(a) shows a transmission image containing a mixture of the three types of plastic beads in the liquid paraffin. Other than slight differences in size, the beads are indistinguishable from each other or even invisible, as will be revealed later.

Once again CMA-ES is used to optimize the phase of the pump and probe pulse, using 200 generations, with 10 parents and 20 individuals per generation. For every trial phase profile $\phi(\omega)$, the difference in CARS signal generated by a pulse with phase $\phi(\omega)$ and a pulse with phase $-\phi(\omega)$ is measured for each of the substances. As fitness value, we use the difference intensity for the compound of interest minus the maximum difference signal of the other compounds times a scaling factor. The fitness for selective excitation of PMMA is shown in equation 5.2.

$$
\begin{array}{r}
F=\Delta I_{P M M A}-\alpha * \max \left(\Delta I_{P S}, \Delta I_{P E}, \Delta I_{\text {Paraffin }}\right), \\
\Delta I=|I(\phi(\omega))-I(-\phi(\omega))|
\end{array}
$$

The scaling factor is used to compensate for differences in scattering cross section and is chosen to be between 1.5 and 3, where compounds with a strong unshaped CARS signal have a higher scaling factor. A higher scaling factor prevents the algorithm from moving towards solutions which increase both the difference signal for the compound of interest and the difference signal for other (weaker scattering) compounds. For compounds with a lower cross section, a too high scaling factor causes the algorithm to push all difference intensities to zero and become stuck in noise.

Instead of subtracting the difference intensities of all the compounds that are being suppressed, we subtract only the maximum of the difference intensities, reducing the influence of noise in the measurements on the fitness.

A separate optimization is run for each compound, to find a phase profile which selectively excites that compound while suppressing the 
(a) Transmission

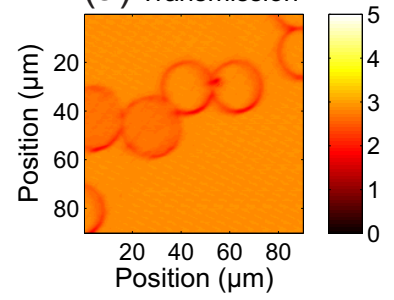

(d) PMMA

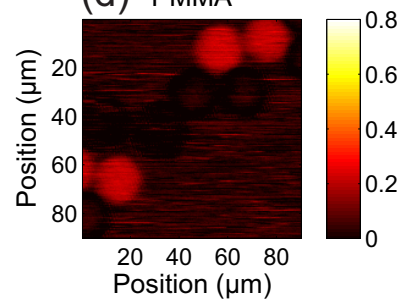

(b) Unshaped

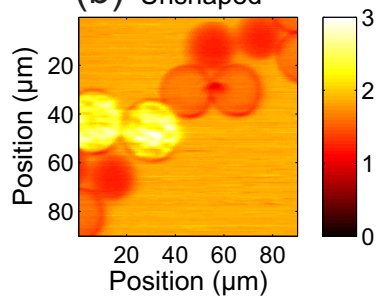

(e) $\mathrm{PE}$

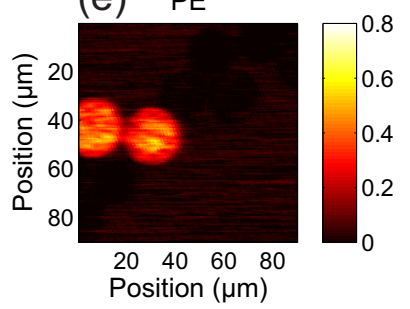

(c) Paraffin
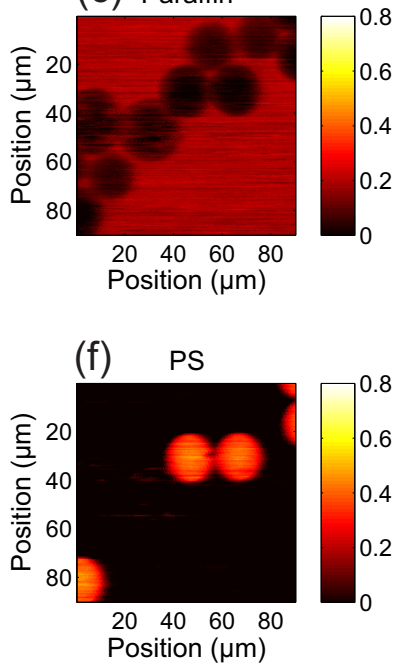

Figure 5.11: Selective imaging in a sample with four resonant compounds. (a) Transmission image. (b) Image taken with an unshaped excitation pulse. (c) Difference image taken with paraffin selective phase. (d) Difference image taken with PMMA selective phase. (e) Difference image taken with polyethene selective phase. (f) Difference image taken with polystyrene selective phase. 
signals from the other resonant compounds in the sample. This set of optimizations is performed on the same sample region. The resulting phase profiles are used for chemically selective imaging of the sample, as shown in figure 5.11.

As can be seen in figure 5.11(b), there is CARS signal from all four components in the sample when using an unshaped pulse. Although it is now possible to see differences in the beads, it is not trivial to identify them according to their chemical composition. Furthermore, it is now clear that the transmission image (figure 5.11(a)) gives incomplete information, as some of the beads are (almost) invisible.

In the difference images made with the optimized selective phase shapes (figure 5.11(c-f)), the individual compounds are easily identifiable, while the signal from the other compounds is clearly suppressed. The contrast ratio varies depending on the compound of interest and ranges from 4:1 (for paraffin) to 13:1 (for polystyrene).

Figure 5.12 shows chemically selective imaging in a sample with five resonant compounds. Here the same approach is used as described for the four compound sample, where a separate optimization is performed for each individual compound. The sample consists of $20 \mu \mathrm{m}$ PMMA microspheres, $20 \mu \mathrm{m}$ polystyrene (PS) microspheres, 20-27 $\mu \mathrm{m}$ polyethene (PE) microspheres, and $12 \mu \mathrm{m}$ melamine resin microspheres, dried on a glass coverslide. The dried beads are once again covered with a layer $( \pm$ $200 \mu \mathrm{m})$ of liquid paraffin and another coverslide on top.

In figure 5.12(a) the CARS signal for an unshaped pulse is shown, where all the compounds in the sample show up, and it is not trivial to distinguish them from each other. In figure 5.12(b-f) the difference CARS images show the individual compounds in the sample while suppressing the other resonant contributions. The contrast has decreased compared to the four compound sample, but the different compounds are still clearly distinguishable. The contrast in this case ranges from 2:1 (for paraffin) to 10:1 (for polystyrene). 
(a) Unshaped



(d) Melamin

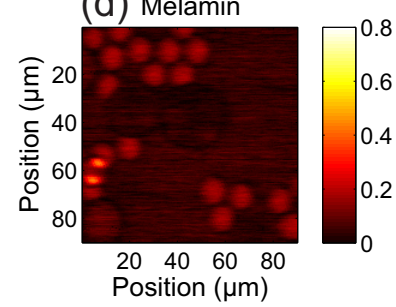

(b) Paraffin

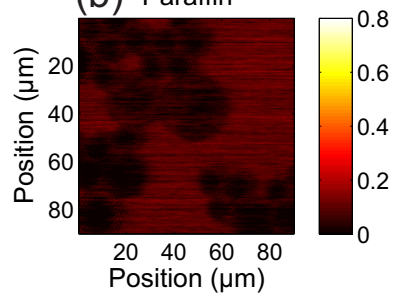

(e) $\mathrm{PE}$

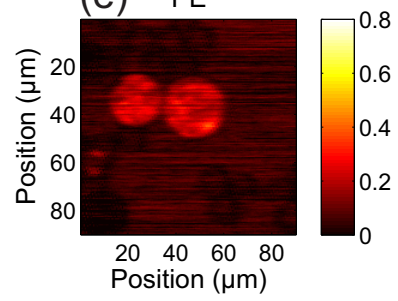

(c) PMMA

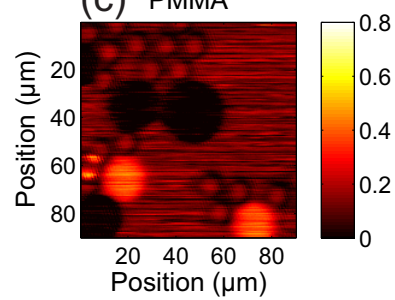

(f) PS

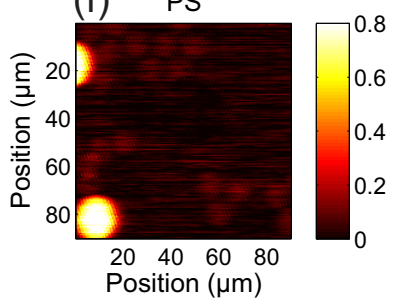

Figure 5.12: Selective imaging in a sample with five resonant compounds. (a) Image taken with an unshaped excitation pulse. (b) Difference image taken with paraffin selective phase. (c) Difference image taken with PMMA selective phase. (d) Difference image taken with melamin selective phase. (e) Difference image taken with polyethene selective phase. (f) Difference image taken with polystyrene selective phase. 


\subsection{Discussion}

In this chapter, chemically selective imaging using complex phase profiles has been shown. These phase profiles are obtained using CMA-ES, analogous to the theoretical study in chapter 4 . The results in this chapter show promise for non-resonant background free imaging of samples with many different compounds, such as cells or other biologically relevant samples, with improved selectivity and contrast.

The obtained contrast ratio in the experiments shown in this chapter, however, is lower than predicted in chapter 4 . This lower contrast ratio has a number of causes. In chapter 4 it has been shown that phase noise has a notable effect on the obtainable contrast ratio. Imperfections in the applied phase shape due to calibration errors in the SLM or residual phase on the pump and probe pulse will reduce the contrast ratio.

Furthermore, the contrast ratio in the experiment is limited by noise, as it is impossible to suppress the CARS difference signals to absolute zero. There is noise from dark counts on the detector and from the electronics, but the main source of noise is intensity noise of the Ti:Sapphire oscillator. The pump and probe pulse has an intensity noise on the order of $3 \%$ at low frequencies $(<10 \mathrm{~Hz})$, which gives an intensity noise of about $6 \%$ on the CARS signal, due to the quadratic power dependence on the pump beam in our setup. The amount of noise in the difference image is even bigger due to our subtraction scheme to obtain the difference image. Therefore it is expected that the obtainable contrast ratio in the experiment can be considerably improved by a more stable pump laser.

Another issue of the current setup is the speed of both the evolutionary optimization and the imaging. The transimpendance amplifier, which is used to amplify the CARS signal that is picked up by the photodiode, determines the maximum modulation frequency due to its limited bandwidth $(3.37 \mathrm{kHz})$. The Stokes beam is currently modulated by a chopper wheel at $327 \mathrm{~Hz}$, which forces the integration time on the lock-in amplifier to values above 10 milliseconds. By incorporating an acousto-optical modulator (AOM) in the Stokes beam, in combination with direct lock-in detection on the (unamplified) photodiode signal, very high modulation frequencies (up to several $\mathrm{MHz}$ ) are possible. This decreases both the minimum integration time on the lock-in amplifier, as well as other noise picked up by the photodiode. 
Because the optimization is only run for 150 or 200 generations in the experiment, it is not guaranteed to have converged to the global optimum yet. However, running longer optimizations is challenging due to slow drift in the alignment of the system. The system slowly misaligns over the course of several hours, which disturbs any long-term optimizations.

Another point of improvement has to do with the calibration of the SLM. The SLM is calibrated on a per pixel basis and the resulting lookup table for the drive levels is big ( \pm 50000 values). As a result, translating phase profiles to drive levels is a time consuming step in the optimization. By using a more efficient drive level lookup scheme, the optimization speed can be improved significantly, allowing optimizations to run for more generations, which can improve contrast.

The proof of principle experiments presented in this chapter herald a bright future for label-free chemically selective imaging. Potential applications range from non-invasive biomedical imaging to novel material characterization and high throughput screening. The utilization of more complex optimization goals, such as the detection of simultaneous presence of several compounds in the focal volume, or the mapping of conformational differences, has the potential to open up an even wider range of interesting applications. 


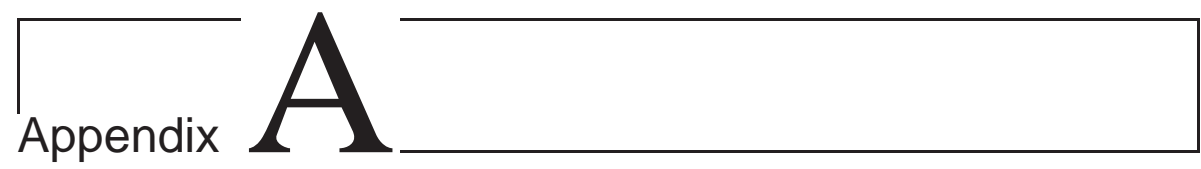

SLM calibration 


\section{A.1 SLM calibration}

\section{A.1.1 Introduction}

In this appendix, a number of calibration and verification techniques are discussed, for a phase-only SLM (A.1.2) and a phase and amplitude SLM (A.1.3). The calibration techniques described in this section are based on earlier work by Dr. Sytse Postma [56]. The techniques described here are aimed at the calibration of a 1D SLM that is used in a 4-f zero-dispersion spectral phase shaping setup [86]. Even though these techniques are primarily intended for liquid crystal based SLMs, they can also be useful for other pixel-based SLM devices, such as MEMSbased pulse shapers.

Liquid crystal based SLMs operate by applying a voltage over a birefringent liquid crystal layer. The effect depends on the type of liquid crystal and the orientation of the liquid crystals. There can be a re-orientation of the crystal layer, which influences the effective refractive index for certain polarizations [90], or the polarization of the incident light itself can be rotated by the liquid crystal layer [91]. The voltage that is applied to the liquid crystal layer typically has a range of 0-10 Volt and this voltage range is typically evenly divided into a number of discrete steps (also called drive levels or grey levels), with which the SLM can be controlled. The number of drive levels can range from 7 bits (128 levels) [90] to 12 bits (4096 levels)[91].

For the SLMs used in this work, there is no straightforward correlation between the applied voltage and the resulting phase and/or amplitude modulation (see figure A.9). Therefore it is important to obtain a calibration that accurately describes the relation between the applied voltage (drive level) and the resulting phase and/or amplitude modulation. Furthermore, the birefringence of the liquid crystal is a function of wavelength, which has to be taken into account. There can also be non-uniformity between the pixels of the SLM, requiring a pixel-based calibration method.

\section{A.1.2 Phase-only SLMs}

In the case of a phase-only SLM, it is not very straightforward to calibrate the SLM using intensity measurements, since the change in phase has no effect on the intensity of the fundamental. It is possible to calib- 




Figure A.1: Schematic of a setup for second harmonic generation calibration of a spatial light modulator. $C M=$ Curved Mirror, SLM = Spatial Light Modulator, $M=$ Mirror, OBJ = Microscope Objective, $S H G=$ Second Harmonic Generation crystal, $F=$ Filter that rejects the fundamental and transmits the $S H G$ signal, $F M=$ Flip Mirror, $P D=$ Photodiode.

rate the SLM using an interferometric approach [92, 93]. One can also rely on nonlinear processes to detect phase changes. Here we use second harmonic generation, and detail its use in the calibration of a phaseonly SLM. The setup for calibration of a phase-only SLM using second harmonic generation is shown in figure A.1.

The calibration is performed by applying a phase modulation at a small number of adjacent pixels of the SLM. The depth of this phase step is scanned from minimum to maximum modulation, and the second harmonic spectrum generated by the phase shaped light is recorded. This process is repeated for each neighbouring set of pixels, until the entire SLM has been covered. The measured second harmonic spectrum and integrated second harmonic spectrum of such a phase scan for one set of 100 pixels near the center wavelength (maximum intensity) of the pulse is shown in figure A.2.

It can be seen from figure A.2 that the intensity of the second harmonic oscillates as a function of the applied phase modulation. The intensity of the second harmonic is lowest when the subset is $\pi$ radians out of phase with the rest of the spectrum (see figure A.3), and highest when it is in phase with the rest of the spectrum. There is a cosine square relation between the second harmonic intensity and the applied phase modulation, which is described in equation A.1. There is a maximum when there is no phase modulation and the entire spectrum is in phase. 
(a)

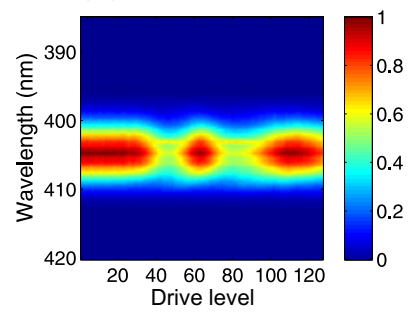

(b)

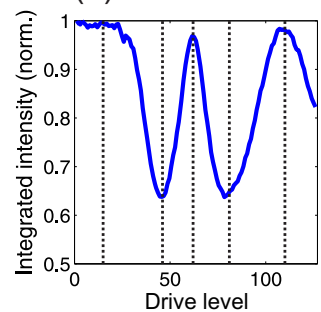

(c)



Figure A.2: (a) Second harmonic spectrum and (b) integrated and normalized second harmonic spectrum as a function of drive level for a phase scan on a subset of (100) pixels of the SLM. The dashed lines indicate the points along which the graph is segmented for the calibration. (c) shows the resulting calibration curve, showing phase modulation as a function of applied drivelevel.

$$
I_{S H G} \propto 1-\alpha \cos ^{2}\left(\frac{\phi}{2}\right)=1-\frac{\alpha *(1+\cos (\phi))}{2}
$$

where $I_{S H G}$ is the intensity of the second harmonic, $\alpha$ is a scaling factor which depends on the width of the subsection compared to the full pulse width, and $\phi$ is the applied phase modulation in radians.

To obtain the relation between drive level and phase modulation, the trace in figure A.2(b) is smoothed using a moving window average over 11 data points, renormalized between -1 and 1 and the maxima and minima are determined. The trace is then segmented along these maxima and minima (indicated by the dashed lines in figure A.2(b))and for every segment, the resulting phase modulation is calculated using equation A.1. The resulting phase modulations for every segment are then stitched together, using a $\pi$ radian phase offset between every segment. The resulting curve of phase modulation as a function of drive level is shown in figure A.2(c).

The relation between pixel number and wavelength can not be directly obtained from the measurements on the subset of pixels, as the effect of the phase modulation is smeared out in the second harmonic process (which can be described as a convolution of the pulse with itself). To determine this relation, we scan a $\pi$ radians modulation step over the mask. This means that initially all pixels of this mask start out at minimum modulation. The first pixel is then switched to $\pi$ radians mod- 


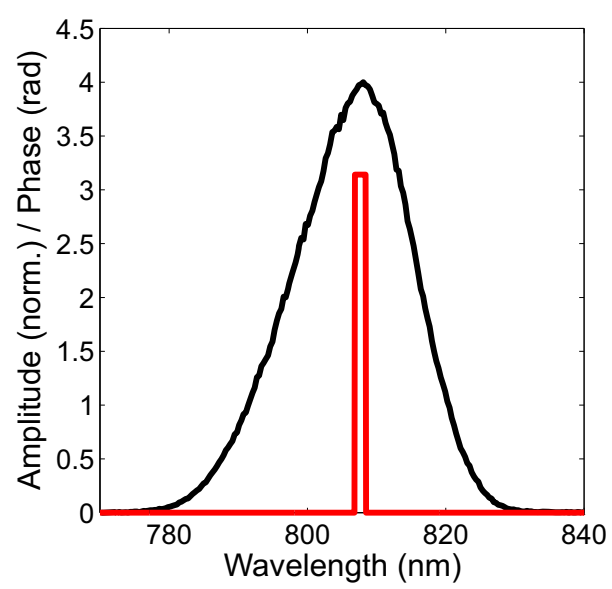

Figure A.3: Fundamental spectrum (black) and applied phase (red). A subset of 100 pixels has a $\pi$ phase difference with the rest of the spectrum.

ulation and the second harmonic spectrum is measured. The following pixel is then switched over to a $\pi$ radian modulation, after which the spectrum of the shaped light is measured. This procedure is repeated until all pixels of the SLM have been switched over, after which there will be a $\pi$ radians modulation over the entire mask. The measured second harmonic spectra have a characteristic (triangular) peak, that is offset from the center of the spectrum by twice the offset of the phase step on the SLM from the center (maximum intensity) of the fundamental spectrum. If the phase step on the SLM is in the center (maximum intensity) of the fundamental spectrum, the characteristic peak is in the center of the SHG spectrum as well. This effect is shown in figure shown in figure A.4. By detecting the location of this characteristic peak in the second harmonic spectrum, the wavelength at which the phase step on the SLM is, can be determined. After a wavelength has been determined for every pixel by peak detection, a second order polynomial function that relates wavelengths to pixel numbers is constructed, as shown in figure A.5.

\section{A.1.3 Phase and amplitude SLMs}

Using a dual-mask configuration, it is possible to obtain both amplitude and phase modulation from a single spatial light modulator. Such a phase and amplitude SLM generally consists of two liquid crystal layers (masks), that can be individually controlled with their own set of driving voltages. 

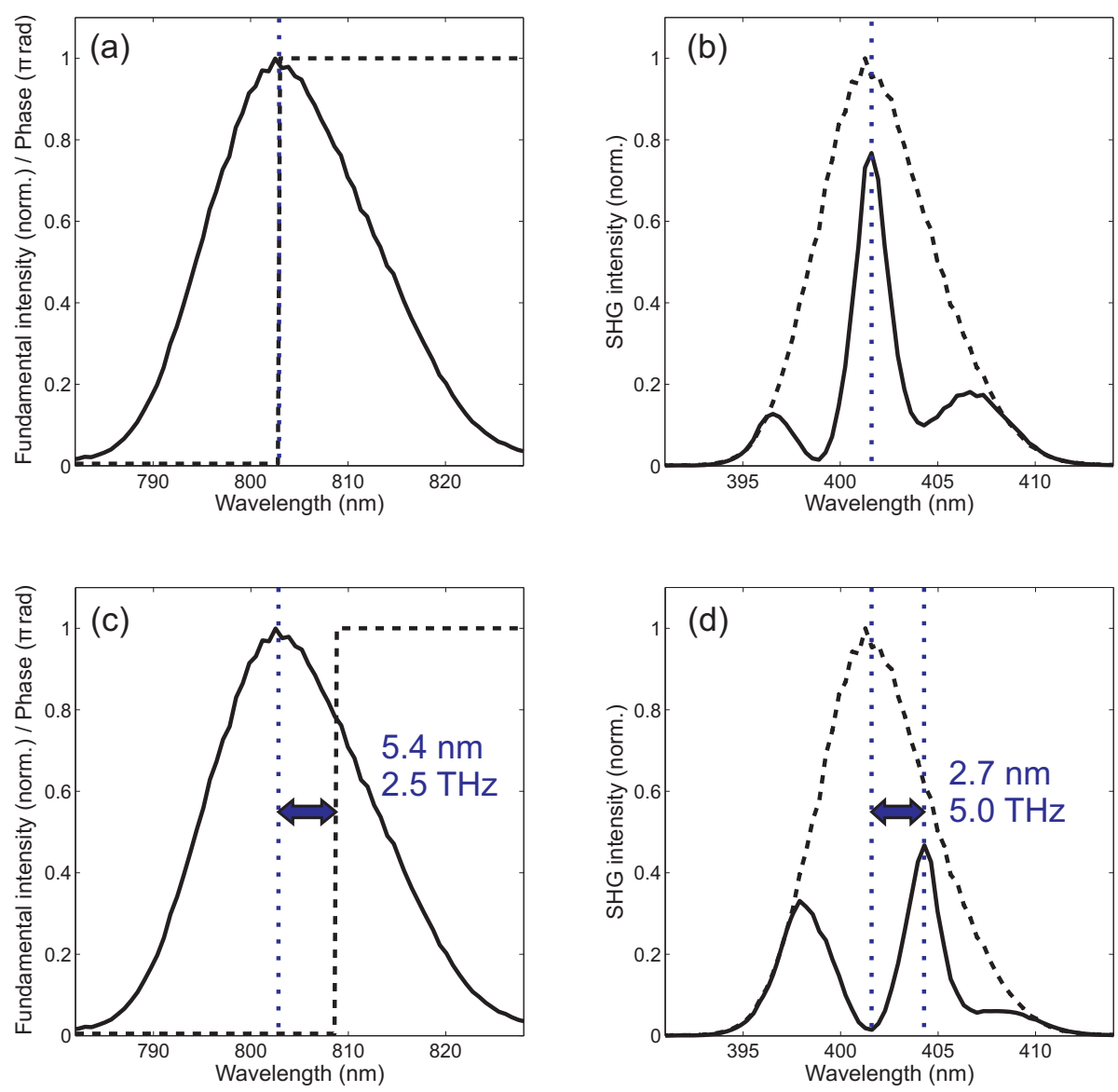

Figure A.4: The effect of a $\pi$ phase step for second harmonic generation. (a) shows the fundamental intensity spectrum (solid) with a $\pi$ phase step in the center of the spectrum (dashed) and (b) shows the corresponding second harmonic spectrum with (solid) and without (dashed) phase step in the fundamental. (c) shows the same fundamental intensity spectrum (solid) as in (a), but with a $\pi$ phase step that is offset from the center of the spectrum (dashed) and (d) shows the corresponding second harmonic spectrum with (solid) and without (dashed) phase step in the fundamental. In blue is indicated how the shift of the peak in the second harmonic spectrum is related to the shift of the phase step in the fundamental. 




Figure A.5: $S H G$ wavelength calibration, showing the retrieved frequencies for different pixels (black) and the second order fit (red) that is used to describe the relation between pixel number and frequency.

Amplitude modulation is achieved by a rotation of the polarization in the liquid crystal layers and filtering the output with a polarizer in front of the SLM. Hence, the amplitude modulation is proportional to the cosine of the total polarization rotation in the SLM, which is equal to the sum of the polarization rotation in the individual liquid crystal layers (equation A.2). Minimum transmission occurs when the polarization is rotated by $90^{\circ}$ degrees.

$$
\Delta I \propto \cos \left(\Delta P_{\text {mask }_{1}}+\Delta P_{\text {mask }_{2}}\right)
$$

where $\Delta I$ is the modulation of the intensity of the laser beam, and $\Delta P_{\text {mask }_{1}}$ and $\Delta P_{\text {mask }_{2}}$ describe the rotation of the polarization by the first and second liquid crystal mask respectively.

Phase modulation is obtained by rotation of the polarization in the first crystal layer, which leads to a change in effective refractive index, due to the birefringence of the liquid crystal layer. This change in the effective refractive index affects the optical path length and hence the phase delay that is applied to the light. The polarization is subsequently rotated back to its original state by the second liquid crystal layer. The phase delay is thus proportional to half the difference in polarization rotation between the two masks: 


$$
\Delta \phi \propto \frac{\Delta P_{\text {mask }_{1}}-\Delta P_{\text {mask }_{2}}}{2}
$$

where $\Delta \phi$ is the phase modulation that is applied to the laser beam, and $\Delta P_{\text {mask }_{1}}$ and $\Delta P_{\text {mask }_{2}}$ describe the rotation of the polarization by the first and second liquid crystal mask respectively.

In the case of the CRi SLM that was used, the extraordinary axes of the liquid crystal layers are oriented orthogonally with respect to each other and at a $45^{\circ}$ angle with respect to the (p-)polarization of the incoming light [91]. Hence, when applying similar driving patterns to both masks, the polarization rotation of the first mask is compensated by the second mask and the phase modulation can be described as the average of the two driving patterns:

$$
\Delta \phi \propto \frac{\Delta D_{\text {mask }_{1}}+\Delta D_{\text {mask }_{2}}}{2}
$$

where $\Delta \phi$ is the applied phase modulation, and $\Delta D_{\text {mask }_{1}}$ and $\Delta D_{\text {mask }}$ describe the driving patterns (in radians) for the first and second mask respectively.

In the case of orthogonally aligned liquid crystal axes, maximum amplitude modulation occurs when both masks have opposite driving patterns, and the amplitude modulation can be described as:

$$
\Delta I \propto \cos ^{2}\left(\frac{1}{2}\left(\Delta D_{\text {mask }_{1}}-\Delta D_{\text {mask }_{2}}\right)\right)=\frac{1+\cos \left(\Delta D_{\text {mask }_{1}}-\Delta D_{\text {mask }_{2}}\right)}{2}
$$

where $\Delta I$ is the intensity modulation, and $\Delta D_{\text {mask }_{1}}$ and $\Delta D_{\text {mask }_{2}}$ describe the driving patterns (in radians) for the first and second mask respectively.

From equations A.4 and A.5 it can be seen that the amplitude modulation and phase modulation are related to each other. Therefore, one can use the amplitude modulation to individually calibrate the liquid crystal masks. The calibration is performed by keeping one of the masks at zero modulation. The drive level of the other mask is swept from 0 to 4095, maintaining the same drive level across all pixels, and the spectrum of the fundamental light is measured for each drive level. This measurement is performed for both masks, in order to obtain separate calibrations for each mask, since their behaviour differs significantly. An 

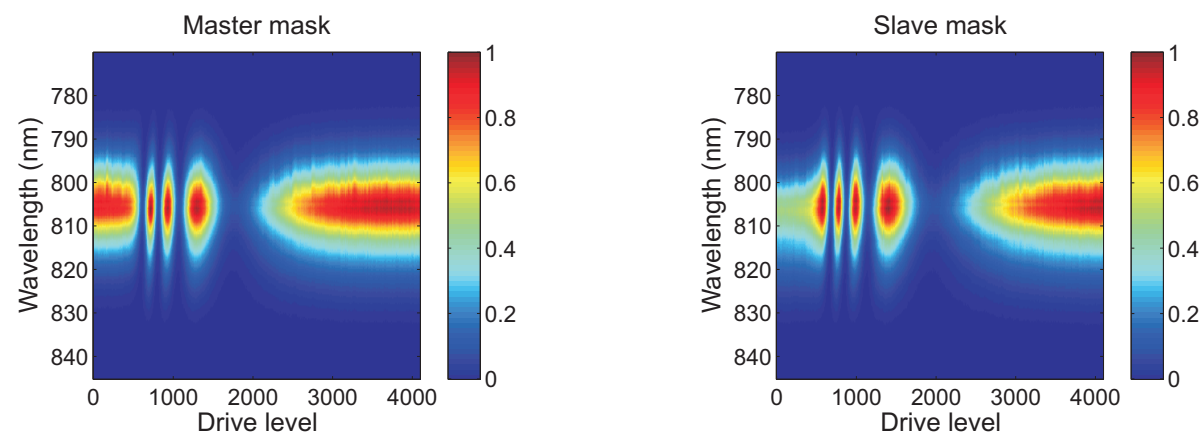

Figure A.6: Measured (normalized) fundamental spectrum as a function of drive level for the master mask (left) and slave mask (right).

example of such a calibration measurement is shown in figure A.6.

The measured intensity as a function of drive level is plotted for each measured wavelength, as shown in figure A.7. To reduce the influence of noise on the calibration a moving window average is subsequently applied to this spectrum. After this averaging, the maxima and minima of the trace are determined. From formula A.5 it is apparent that every subsequent maximum or minimum equals a $\pi$ radians increase in modulation. The trace is segmented along the maxima and minima and rescaled to range from -1 to 1 . The applied modulation (in radians) is then calculated from the measured intensity modulation using equation A.5. The individual segments are subsequently stitched together, using a $\pi$ offset from the previous segment, and a lookup table is constructed that relates the drive level for each pixel of both masks to the applied modulation.

The relation between the wavelength of the incoming light and the pixel numbers on the SLM can be obtained either by the same means as described in chapter A.1.2, or by means of amplitude shaping, as will be explained below.

In the case of phase and amplitude SLMs, one can easily characterize the relation between the wavelength of the incoming light and the pixel numbers of the SLM by applying an amplitude shaping pattern. For the CRi SLM that we are considering here, the intermask misalignment is specified as $2 \mu \mathrm{m}$, which is very small compared to the pixel pitch of $100 \mu \mathrm{m}$ [91]. It is therefore expected that the relation between incident wavelength and pixel number is the same for both masks. In this case, 




Figure A.7: Measured normalized intensity of the fundamental light after the SLM as a function of the drive level that is applied to the pixels of the master mask. The slave mask is kept at minimum modulation (4095 drive level) for all pixels.

it is sufficient to characterize this relation for only one of the masks in the SLM. If the ratio between misalignment of the masks and the pixel pitch is significant, it will be necessary to do this calibration for both masks individually.

For the wavelength calibration, one of the masks is kept at minimum modulation for all pixels. On the other mask, a $\pi$ radians modulation step is scanned over the mask, switching the pixels over to $\pi$ radians modulation depth sequentially, and the fundamental spectrum is measured after each pixel switch. The resulting spectra are shown in figure A.8.

The relation between the wavelength of the incident light and the pixel numbers of the SLM is determined by determining the cut-off wavelength for a number of pixels from figure A.8. The complete relation is then obtained by fitting these data points with either a linear or quadratic function. Subsequently, the lookup table of phase modulation as a function of wavelength and drive level is constructed, as shown in figure A.9. 


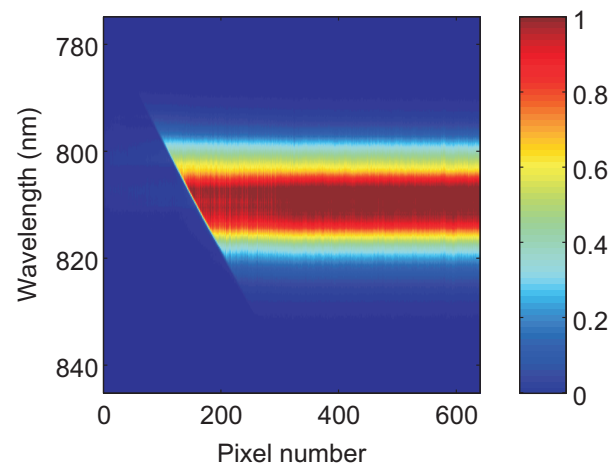

Figure A.8: Normalized measured fundamental spectra as a function of the pixel number where the $\pi$ radians modulation step occurs.

(a)

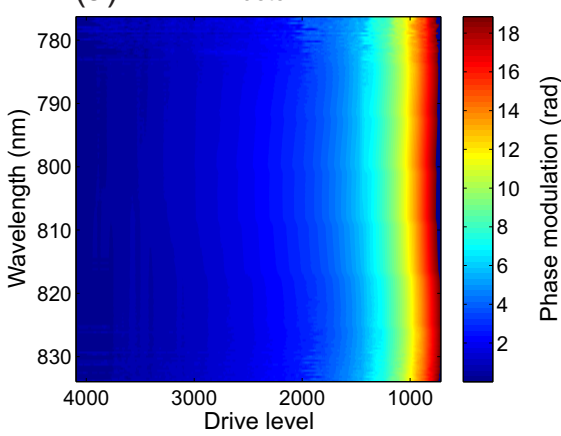

(c)

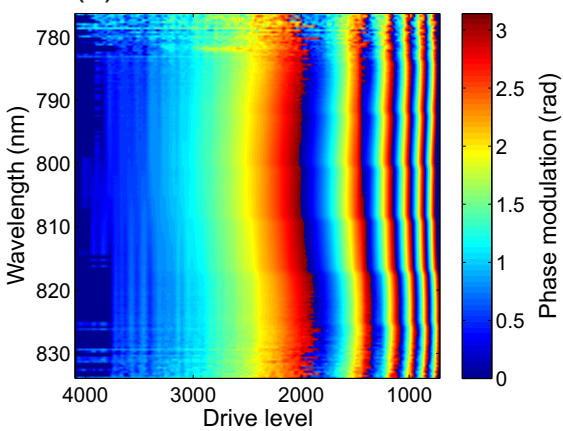

(b)

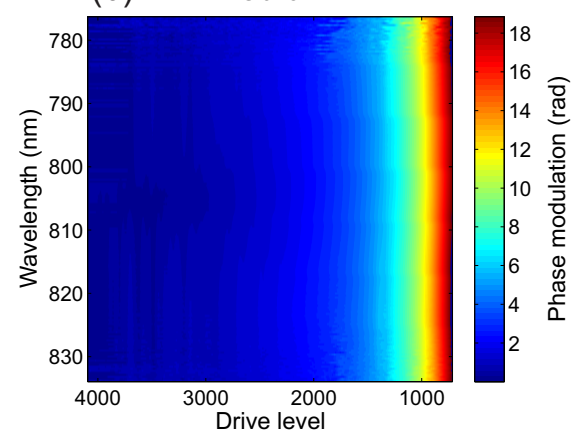

(d)

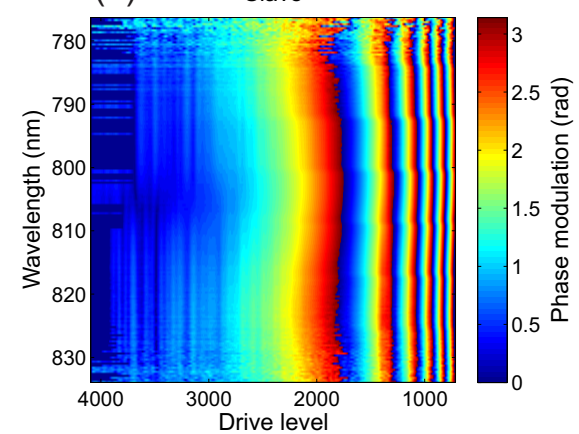

Figure A.9: Example of an SLM calibration. (a,b) Phase modulation as a function of drive level (horizontal) and wavelength (vertical). (c,d) The phase modulation as shown in (a,b), modulo $\pi$. (a,c) shows the calibration for the master mask and $(b, d)$ shows the calibration for the slave mask. 


\section{A.2 SLM calibration verification}

In this section a number of checks to verify the validity of the calibration and diagnose problems are discussed. These techniques use either the fundamental light or the second harmonic of the fundamental to verify the calibration. These methods are designed to look at phaseonly shaping, where amplitude shaping is considered to be undesirable. It is important to consider dispersion in the setup, in order to make sure that the spectral phase of the laser pulse at the sample matches with the intended phase profile. Any additional dispersion in the system should be avoided, for example by using reflective optics instead of transmissive optics. To determine if there is any residual dispersion in the system, one can use for example autocorrelation or frequency resolved optical gating (FROG). Second and third order residual phase can be compensated by a prism compressor and detuning of the grating(s) in the 4-f shaping setup. Higher order residual phase has to be compensated by the SLM. Large amounts of residual second or third order phase may indicate a misalignment in the distance between the grating and the focusing element in the 4 -f setup.

\section{A.2.1 Fundamental light}

One direct check is the measurement of the (shaped) fundamental light. The intensity of the fundamental should remain constant, regardless of the applied phase profile. In practice this does not hold true for most phase shaping designs, as high frequency modulations (such as sharp phase steps) scatter light out of the system. For smooth phase profiles this is still a reasonable assumption however. Figure A.10(a) shows the spectrum of the fundamental for a scan of a constant phase offset that increases in depth. The relative deviation (figure A.10(b)) is obtained by dividing the measured spectra by the mean spectrum of all obtained spectra. Any deviations in the spectrum or power indicate imperfect phase-only shaping or a discrepancy between the desired phase profile and the applied phase profile.

\section{A.2.2 Second harmonic generation}

Although the fundamental light provides amplitude information, little information can be obtained about the correctness of the applied phase 
(a)

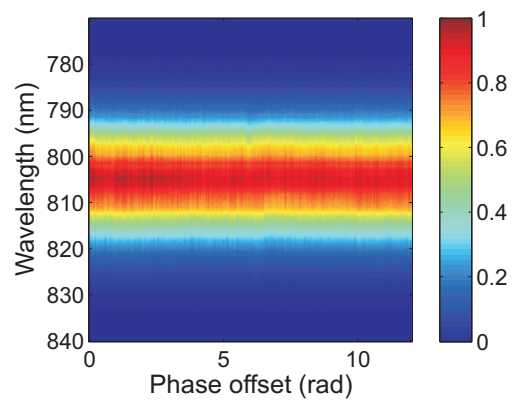

(b)

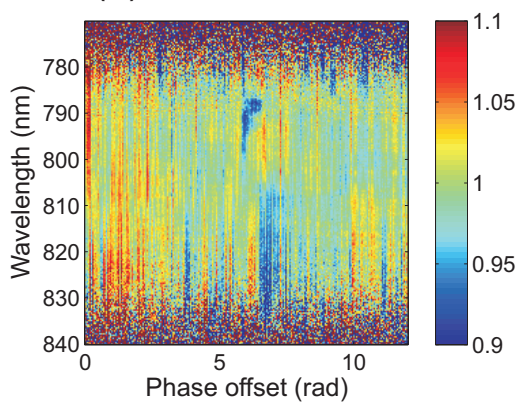

Figure A.10: Constant phase offset scan using the fundamental light. (a) shows the measured fundamental spectrum for different levels of phase offset and (b) shows the spectra in (a) divided by the mean of all the measured spectra.

profiles. Second harmonic generation (SHG) was used to verify the calibration. Since SHG uses two photons from the same laser pulse, it is insensitive to constant or linear spectral phase of the pulse and it is only the second and higher order differences in spectral phase that influence the SHG process.

Figure A.11 shows the SHG spectrum for a scan of a constant phase offset that increases in depth. The intensity and spectrum should not change with the applied phase offsets. Any change in either the spectrum or the intensity of the SHG indicates a problem with either the SLM itself or the calibration of the SLM.

Another check that was performed is scanning a $\pi$ phase step through the fundamental spectrum and measuring the second harmonic spectrum. Scanning a positively or negatively sloped phase step through the fundamental spectrum, yields a very distinct peak in the second harmonic spectrum, as discussed in chapter A.1.2. The SHG spectrum is plotted as a function of the phase step position, as shown in figure A.12. This 2D spectrum displays mirror-symmetry around the diagonal line in the center, which has a slope of two in frequency space.

\section{A.2.3 Autocorrelation}

An intensity autocorrelation can be used to determine the pulse length for simple pulses. In combination with a measurement of the funda- 
(a)

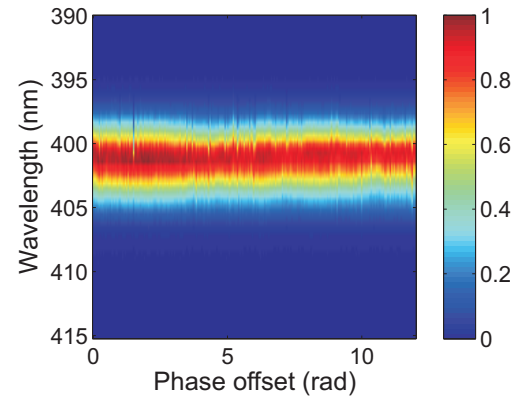

(b)

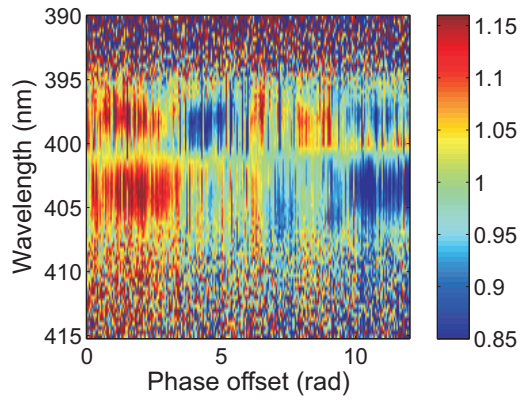

Figure A.11: Constant phase offset scan using second harmonic generation. (a) shows the measured second harmonic spectrum for different levels of phase offset and (b) shows the second harmonic spectra in (a) divided by the mean of all the measured spectra.

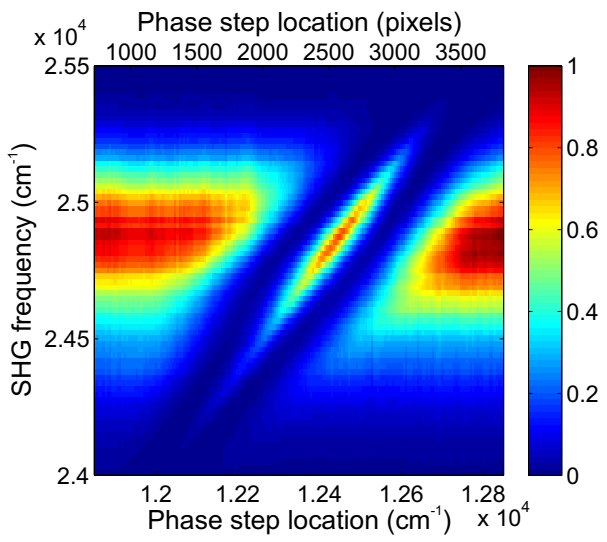

Figure A.12: Second harmonic generation spectrum (vertical) as a function of the location of a $\pi$ phase step (horizontal) that is scanned through the fundamental spectrum. 


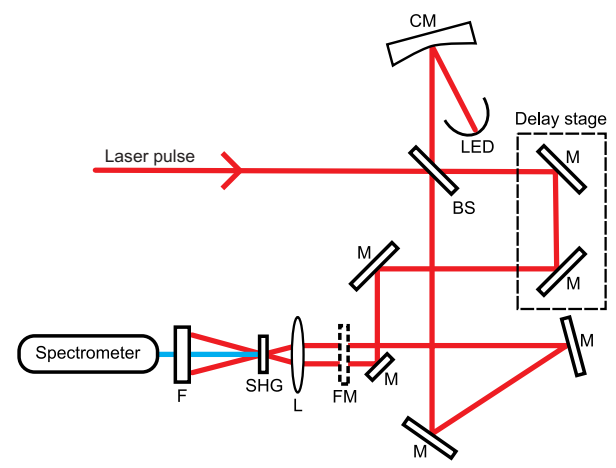

Figure A.13: Schematic drawing of an autocorrelation and FROG setup. $B S=50 / 50$ Beamsplitter, $C M=$ Curved Mirror, LED $=$ Light Emitting Diode, $M=$ Mirror, FM=Flip Mirror, $L=$ Lens, $S H G=$ Second Harmonic Generation crystal, $F=$ Filter that rejects the fundamental and transmits the SHG signal.

mental spectrum, the actual pulse length and shortest possible pulse length for the given spectral bandwidth can be compared. It can provide a rough indication of the amount of temporal broadening of the pulse due to spectral phase distortion [94]. For a complete retrieval of both the phase and amplitude of the pulse, frequency resolved optical gating (FROG) was used. A schematic of a setup for autocorrelation and FROG measurements is shown in figure A.13.

The setup in figure A.13 can be used as an autocorrelator by introducing the flip mirror into the beam. The laser light is focused on a LED, which has a shorter emission wavelength than the laser pulse. Because the emission of the LED is at a shorter wavelength, the band gap energy of the LED is higher than the energy of a single photon of the laser pulse. The band gap can be bridged using a two-photon absorption, turning the LED into a two-photon detector for the autocorrelator.

\section{A.2.4 Frequency resolved optical gating}

Frequency resolved optical gating (FROG) allows for the complete retrieval of both phase and amplitude information of ultrashort pulses. FROG was developed by Trebino et al. in 1993 [95] and is frequently used for the characterization of ultrashort pulses. In this work second harmonic generation FROG (SHG-FROG) is used. Because SHG-FROG 
relies on a second order nonlinearity, it is able to measure relatively low intensity pulses. Furthermore, detection of the second harmonic signal is relatively straightforward. A disadvantage of SHG-FROG is the ambiguity of the direction of time. This can be resolved with an additional measurement [96].

A schematic of the SHG-FROG setup is shown in figure A.13, where the FROG is measured by taking the flip mirror out of the beam, allowing the parallel beams to be focused into the SHG crystal, generating the FROG signal. By looking at the amplitude and phase profiles of the pulses that are retrieved from the SHG-FROG trace, misalignment of the phase shaping setup or other problems in the system can be identified, as shown in figure A.14. After identifying the dominating order(s) of residual chirp, the chirp can be corrected using the techniques described at the start of section A.2. 
(a)

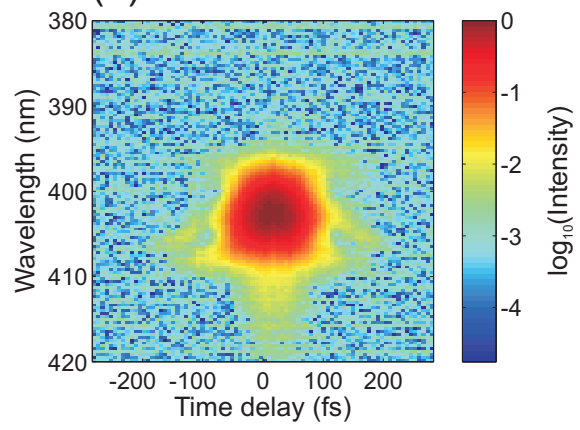

(c)

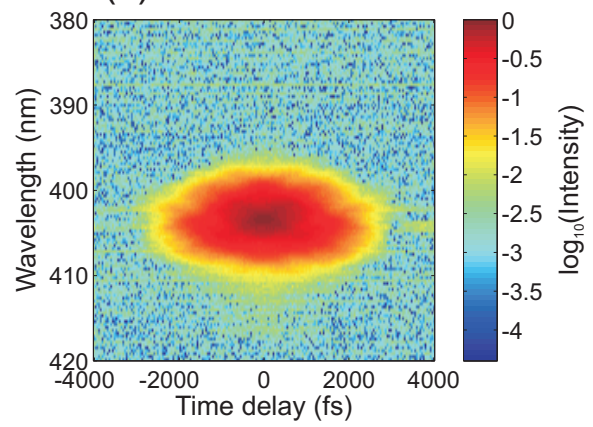

(e)

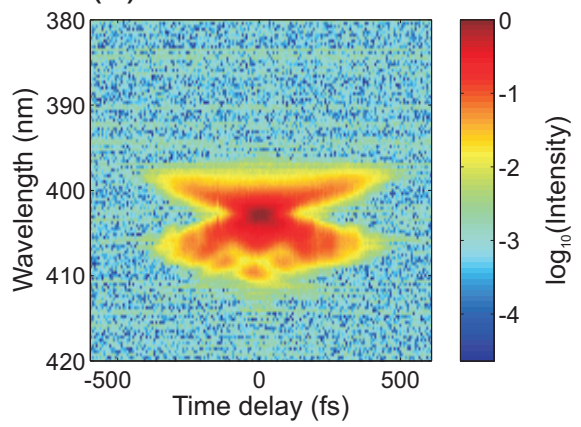

(b)

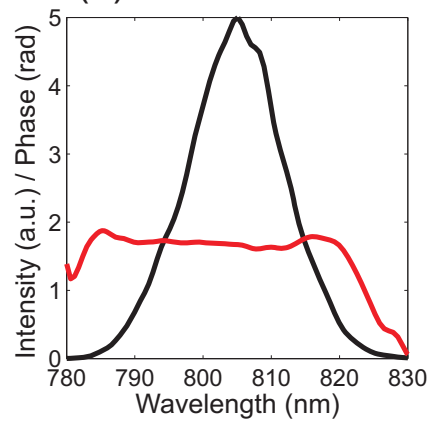

(d)

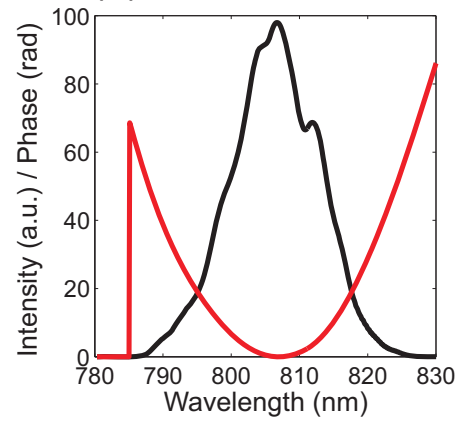

(f)

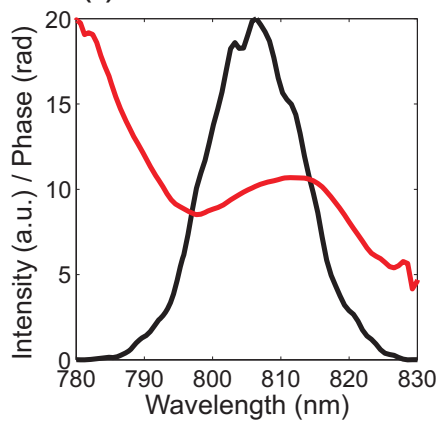

Figure A.14: SHG-FROG measurements for different pulses (left, logarithmic intensity scale) and the retrieved amplitude (right, black) and phase (right, red). (a,b) Near Fourier-limited pulse. (c,d) Pulse with residual second order phase. (e,f) Pulse with residual third order phase. 




Calcite crystal mounts 

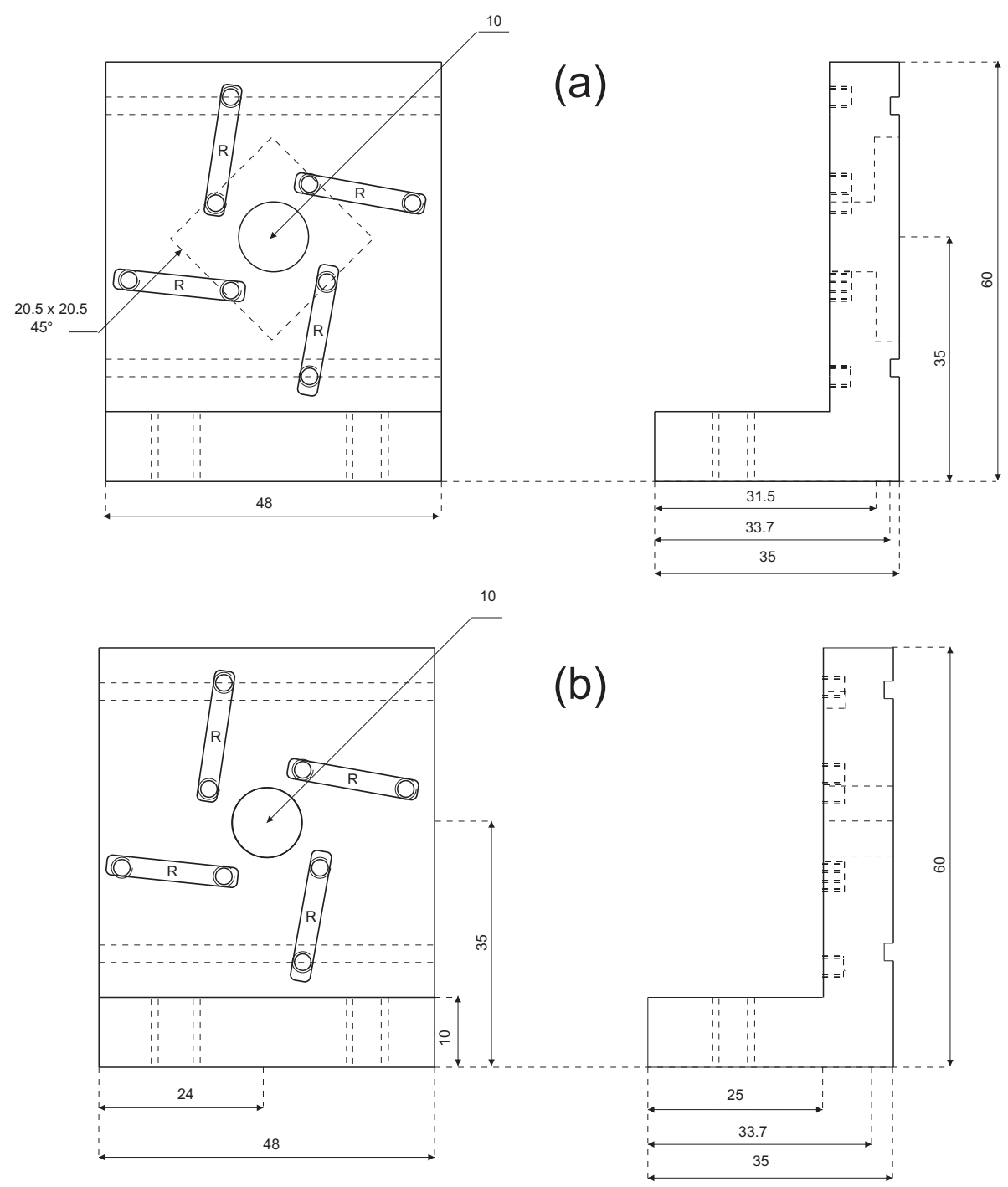

Figure B.1: Schematic of the front and side view of the aluminum mounts for the calcite crystals. The part shown in (a) has a cavity for a calcite crystal in the center. The parts shown in (a) and (b) are screwed together, while the crystal is held in place by a small spring. The resistors used to control the temperature are indicated by the rectangles labeled with an $R$. The two slits underneath and above the crystal are where the temperature sensors are inserted. Sizes are indicated in millimeters. 


\section{Bibliography}

[1] J. W. Strutt, "On the light from the sky, its polarization and colour," Philosophical Magazine 41, 107-120 (1871).

[2] J. W. Strutt, "On the scattering of light by small particles," Philosophical Magazine 41, 447-454 (1871).

[3] G. Mie, "Beiträge zur optik trüber medien, speziell kolloidaler metallösungen," Annalen der Physik 330, 377-445 (1908).

[4] G. Stokes, "On the change of refrangibility of light," Proceedings of the Royal Society of London: Philosophical Transactions of the Royal Society 142, 463-562 (1852).

[5] M. Chalfie, Y. Tu, G. Euskirchen, W. W. Ward, and D. C. Prasher, "Green fluorescent protein as a marker for gene-expression," Science 263, 802-805 (1994).

[6] S. Weiss, "Fluorescence spectroscopy of single biomolecules," Science 283, 1676-1683 (1999).

[7] X. Michalet, A. N. Kapanidis, T. Laurence, F. Pinaud, S. Doose, M. Pflughoefft, and S. Weiss, "The power and prospects of fluorescence microscopies and spectroscopies," Annual Review of Biophysics and Biomolecular structure 32, 161-182 (2003).

[8] C. V. Raman and K. S. Krishnan, "A new type of secondary radiation," Nature 121, 501-502 (1928).

[9] F. Zernike, "Phase contrast, a new method for the microscopic observation of transparent objects," Physica 9, 686-698 (1942).

[10] E. Hecht, Optics (Addison Wesley, San Francisco CA, 2001).

[11] T. H. Maiman, "Stimulated optical radiation in ruby," Nature 187, 493494 (1960). 
[12] W. Denk, J. H. Strickler, and W. W. Webb, "2-photon laser scanning fluorescence microscopy," Science 248, 73-76 (1990).

[13] Y. Barad, H. Eisenberg, M. Horowitz, and Y. Silberberg, "Nonlinear scanning laser microscopy by third harmonic generation," Applied Physics Letters 70, 922-925 (1997).

[14] D. A. Greenhalgh, F. M. Porter, and W. A. England, "The application of coherent anti-Stokes Raman-scattering to turbulent combustion thermometry," Combustion and Flame 49, 171-181 (1983).

[15] R. D. Hancock, K. E. Bertagnolli, and R. P. Lucht, "Nitrogen and hydrogen CARS temperature measurements in a hydrogen/air flame using a near-adiabatic flat-flame burner," Combustion and Flame 109, 323-331 (1997).

[16] T. Lang and M. Motzkus, "Single-shot femtosecond coherent anti-Stokes Raman-scattering thermometry," Journal of the Optical Society of America B 19, 340-344 (2002).

[17] J. D. Miller, M. N. Slipchenko, T. R. Meyer, H. U. Stauffer, and J. R. Gord, "Hybrid femtosecond/picosecond coherent anti-Stokes Raman scattering for high-speed gas-phase thermometry," Optics Letters 35, 2430$2432(2010)$.

[18] J. X. Cheng and X. S. Xie, "Coherent anti-Stokes Raman scattering microscopy: Instrumentation, theory, and applications," Journal of Physical Chemistry B 108, 827-840 (2004).

[19] L. G. Rodriguez, S. J. Lockett, and G. R. Holtom, "Coherent anti-Stokes Raman scattering microscopy: A biological review," Cytometry Part A 69A, 779-791 (2006).

[20] C. W. Freudiger, W. Min, B. G. Saar, S. Lu, G. R. Holtom, C. He, J. C. Tsai, J. X. Kang, and X. S. Xie, "Label-free biomedical imaging with high sensitivity by stimulated raman scattering microscopy," Science 322, 1857-1861 (2008).

[21] C. L. Evans and X. S. Xie, "Coherent anti-Stokes Raman scattering microscopy: Chemical imaging for biology and medicine," Annual Review of Analytical Chemistry 1, 883-909 (2008).

[22] F. Legare, C. L. Evans, F. Ganikhanov, and X. S. Xie, "Towards CARS endoscopy," Optics Express 14, 4427-4432 (2006).

[23] R. W. Boyd, Nonlinear Optics (Academic Press, San Diego CA, 2003).

[24] B. R. Masters and P. T. C. So, Biomedical nonlinear optical microscopy (Oxford University Press, New York NY, 2008).

[25] E. Smith and G. Dent, Modern Raman spectroscopy: A practical approach (John Wiley \& Sons Ltd, Chicester, 1995). 
[26] N. Bloembergen, "The stimulated Raman effect," American Journal of Physics 35, 989-1023 (1967).

[27] P. Nandakumar, A. Kovalev, and A. Volkmer, "Vibrational imaging based on stimulated Raman scattering microscopy," New Journal of Physics 11, 033026 (2009).

[28] P. D. Maker and R. W. Terhune, "Study of optical effects due to an induced polarization third order in the electric field strength," Physical Review 137, A801-A818 (1965).

[29] E. O. Potma, C. L. Evans, and X. S. Xie, "Heterodyne coherent antiStokes Raman scattering (CARS) imaging," Optics Letters 31, 241-243 (2006).

[30] M. Jurna, J. P. Korterik, C. Otto, J. L. Herek, and H. L. Offerhaus, "Background free CARS imaging by phase sensitive heterodyne CARS," Optics Express 16, 15863-15869 (2008).

[31] N. Dudovich, D. Oron, and Y. Silberberg, "Single-pulse coherently controlled nonlinear Raman spectroscopy and microscopy," Nature 418, 512514 (2002).

[32] S. H. Lim, A. G. Caster, and S. R. Leone, "Single-pulse phase-control interferometric coherent anti-Stokes Raman scattering spectroscopy," Physical Review A 72, 041803 (2005).

[33] A. Volkmer, L. D. Book, and X. S. Xie, "Time-resolved coherent antiStokes Raman scattering microscopy: Imaging based on a Raman free induction decay," Applied Physics Letters 80, 1505-1507 (2002).

[34] O. Rubner, M. Schmitt, G. Knopp, A. Materny, W. Kiefer, and V. Engel, "Femtosecond time-resolved CARS spectroscopy on binary gas-phase mixtures: A theoretical and experimental study of the benzene toluene system," Journal of Physical Chemistry A 102, 9734-9738 (1998).

[35] F. Ganikhanov, C. L. Evans, B. G. Saar, and X. S. Xie, "High-sensitivity vibrational imaging with frequency modulation coherent anti-Stokes Raman scattering (FM CARS) microscopy," Optics Letters 31, 1872-1874 (2006).

[36] A. Y. Chikishev, G. W. Lucassen, N. I. Koroteev, C. Otto, and J. Greve, "Polarization sensitive coherent anti-Stokes Raman scattering spectroscopy of the amide I band of proteins in solutions," Biophysical Journal 63, 976-985 (1992).

[37] K. Orsel, E. T. Garbacik, M. Jurna, J. P. Korterik, C. Otto, J. L. Herek, and H. L. Offerhaus, "Heterodyne interferometric polarization coherent anti-Stokes Raman scattering (HIP-CARS) spectroscopy," Journal of Raman Spectroscopy 41, 1678-1681 (2010). 
[38] J. P. R. Day, K. F. Domke, G. Rago, H. Kano, H. Hamaguchi, E. M. Vartiainen, and M. Bonn, "Quantitative coherent anti-Stokes Raman scattering (CARS) microscopy," Journal of Physical Chemistry B 115, 7713-7725 (2011).

[39] D. W. McCamant, P. Kukura, S. Yoon, and R. A. Mathies, "Femtosecond broadband stimulated Raman scattering: Apparatus and methods," Review of Scientific Instruments 75, 4971-4980 (2004).

[40] H. Frostig, O. Katz, A. Natan, and Y. Silberberg, "Single-pulse stimulated Raman scattering spectroscopy," Optics Letters 36, 1248-1250 (2011).

[41] J. X. Chen, A. Volkmer, L. D. Book, and X. S. Xie, "Multiplex coherent anti-Stokes Raman scattering microspectroscopy and study of lipid vesicles," Journal of Physical Chemistry B 106, 8493-8498 (2002).

[42] G. H. W. Wurpel, J. M. Schins, and M. Müller, "Direct measurement of chain order in single phospholipid mono- and bilayers with multiplex CARS," Journal of Physical Chemistry B 108, 3400-3403 (2004).

[43] H. Kano and H. Hamaguchi, "Vibrationally resonant imaging of a single living cell by supercontinuum-based multiplex coherent anti-Stokes Raman scattering microspectroscopy," Optics Express 13, 1322-1327 (2005).

[44] H. A. Rinia, M. Bonn, and M. Müller, "Quantitative multiplex CARS spectroscopy in congested spectral regions," Journal of Physical Chemistry B 110, 4472-4479 (2006).

[45] E. M. Vartiainen, H. A. Rinia, M. Müller, and M. Bonn, "Direct extraction of Raman line-shapes from congested CARS spectra," Optics Express 14, $3622-3630$ (2006).

[46] Y. Liu, Y. J. Lee, and M. T. Cicerone, "Broadband CARS spectral phase retrieval using a time-domain Kramers-Kronig transform," Optics Letters 34, 1363-1365 (2009).

[47] Y. Liu, Y. J. Lee, and M. T. Cicerone, "Fast extraction of resonant vibrational response from CARS spectra with arbitrary nonresonant background," Journal of Raman Spectroscopy 40, 726-731 (2009).

[48] S. A. Zhang, L. Zhang, X. Y. Zhang, L. T. Ding, G. L. Chen, Z. R. Sun, and Z. G. Wang, "Selective excitation of CARS by adaptive pulse shaping based on genetic algorithm," Chemical Physical Letters 433, 416421 (2007).

[49] J. Konradi, A. K. Singh, A. V. Scaria, and A. Materny, "Selective spectral filtering of molecular modes of $\beta$-carotene in solution using optimal control in four-wave-mixing spectroscopy," Journal of Raman spectroscopy 37, 697-704 (2006).

[50] J. Konradi, A. V. Scaria, V. Namboodiri, and A. Materny, "Application of feedback-controlled pulse shaping for control of CARS spectra: the role of phase and amplitude modulation," Journal of Raman spectroscopy 38, 1006-1021 (2007). 
[51] D. Oron, N. Dudovich, D. Yelin, and Y. Silberberg, "Narrow-band coherent anti-Stokes Raman signals from broad-band pulses," Physical Review Letters 88, 063004 (2002).

[52] D. Oron, N. Dudovich, D. Yelin, and Y. Silberberg, "Quantum control of coherent anti-Stokes Raman processes," Physical Review A 65, 043408 (2002).

[53] M. A. van Dijk, M. Lippitz, D. Stolwijk, and M. Orrit, "A common-path interferometer for time-resolved and shot-noise-limited detection of single nanoparticles," Optics Express 15, 2273-2287 (2007).

[54] M. J. Colles, "Efficient stimulated Raman scattering from picosecond pulses," Optics Communications 1, 169-172 (1969).

[55] J. R. Nestor and E. R. Lippincott, "The effect of the internal field on Raman scattering cross sections," Journal of Raman Spectroscopy 1, 305318 (1973).

[56] S. Postma, "Spectral phase shaping for non-linear spectroscopy and imaging," Ph.D. thesis, University of Twente, Enschede, The Netherlands (2008).

[57] S. Zhao, F. Wu, H. Wang, W. Zhong, X. Li, H. Tang, M. Shi, and H. Deng, "Accurate measurement of the main refractive indices and thermo-optical coefficients of the calcite crystal," Chinese Optics Letters 5, 232-234 (2007).

[58] R. T. Dillon and R. G. Dickinson, "Raman spectra from acetone," Proceedings of the National Academy of Sciences of the United States of America 15, 699-702 (1929).

[59] A. F. Pegoraro, A. Ridsdale, D. J. Moffatt, Y. Jia, J. P. Pezacki, and A. Stolow, "Optimally chirped multimodal CARS microscopy based on a single Ti:sapphire oscillator," Optics Express 17, 105744 (2009).

[60] A. C. W. van Rhijn, H. L. Offerhaus, P. van der Walle, J. L. Herek, and A. Jafarpour, "Exploring, tailoring, and traversing the solution landscape of a phase-shaped CARS process," Optics Express 18, 2695-2709 (2010).

[61] M. Jurna, J. P. Korterik, C. Otto, J. L. Herek, and H. L. Offerhaus, "Vibrational phase contrast microscopy by use of coherent anti-Stokes Raman scattering," Physical Review Letters 103, 043905 (2009).

[62] S. H. Lim, A. G. Caster, O. Nicolet, and S. R. Leone, "Chemical imaging by single pulse interferometric coherent anti-Stokes Raman scattering microscopy," Journal of Physical Chemistry B 110, 5196-5204 (2006).

[63] S. H. Lim, A. G. Caster, and S. R. Leone, "Fourier transform spectral interferometric coherent anti-Stokes Raman scattering (FTSI-CARS) spectroscopy," Optics Letters 32, 1332-1334 (2007). 
[64] S. Mukamel, Principles of nonlinear optical spectroscopy (Oxford University Press, New York NY, 1995).

[65] R. Fanciulli, L. Willmes, J. Savolainen, P. van der Walle, T. Bäck, and J. L. Herek, "Evolution strategies for laser pulse compression," Lecture Notes in Computer Science 4926, 219-230 (2008).

[66] N. Hansen, "The CMA evolution strategy: A tutorial," http://www.lri.fr/ hansen/cmatutorial.pdf (2011).

[67] P. Duhamel and M. Vetterli, "Fast Fourier transforms: A tutorial review and a state of the art," Signal Processing 19, 259-299 (1990).

[68] P. van der Walle, H. L. Offerhaus, J. L. Herek, and A. Jafarpour, "Tailoring a coherent control solution landscape by linear transforms of spectral phase basis," Optics Express 18, 973-987 (2010).

[69] P. S. C. Heuberger, P. M. J. van den Hof, and B. Wahlberg, Modelling and identification with rational orthogonal basis functions (Springer, London, 2005).

[70] M. Telescu, N. Tanguy, P. Bréhonnet, and P. Vilbé, "Efficient Grammatrix computation for irrational resonant systems using Kautz models," Signal Processing 87, 3234-3240 (2007).

[71] A. Jafarpour, J. Savolainen, R. de Jong, J. Middag, D. P. Sprünken, P. van der Walle, D. Yang, and J. L. Herek, "Robust orthogonal parameterization of evolution strategy for adaptive laser pulse shaping," Optics Express 17, 11986-12000 (2009).

[72] C. Brif, R. Chakrabarti, and H. Rabitz, "Control of quantum phenomena: past, present and future," New Journal of Physics 12, 075008 (2010).

[73] P. Brumer and M. Shapiro, "Control of unimolecular reactions using coherent light," Chemical Physics Letters 126, 541-546 (1986).

[74] P. Brumer and M. Shapiro, "Laser control of molecular processes," Annual Review of Physical Chemistry 43, 257-282 (1992).

[75] R. Kosloff, S. A. Rice, P. Gaspard, S. Tersigni, and D. J. Tannor, "Wavepacket dancing: Achieving chemical selectivity by shaping light pulses," Chemical Physics 139, 201-220 (1989).

[76] A. Assion, T. Baumert, M. Bergt, T. Brixner, B. Kiefer, V. Seyfried, M. Strehle, and G. Gerber, "Control of chemical reactions by feedbackoptimized phase-shaped femtosecond laser pulses," Science 282, 919-922 (1998).

[77] D. Meshulach and Y. Silberberg, "Coherent quantum control of twophoton transitions by a femtosecond laser pulse," Nature 396, 239-242 (1998). 
[78] T. Chen, A. Vierheilig, P. Waltner, M. Heid, W. Kiefer, and A. Materny, "Femtosecond laser-controlled selective excitation of vibrational modes on a multidimensional ground state potential energy surface," Chemical Physics Letters 326, 375-382 (2000).

[79] B. von Vacano and M. Motzkus, "Molecular discrimination of a mixture with single-beam Raman control," The Journal of Chemical Physics 127, 144514 (2007).

[80] D. Pestov, X. Wang, R. K. Murawski, G. O. Ariunbold, V. A. Sautenkov, and A. V. Sokolov, "Pulse shaping for mode-selective ultrafast coherent Raman spectroscopy of highly scattering solids," Journal of the Optical Society of America B 25, 768-772 (2008).

[81] D. Oron, N. Dudovich, and Y. Silberberg, "Femtosecond phase-andpolarization control for background-free coherent anti-Stokes Raman spectroscopy," Physical Review Letters 90, 213902 (2003).

[82] D. Oron, N. Dudovich, D. Yelin, and Y. Silberberg, "Quantum control of coherent anti-Stokes Raman processes," Physical Review A 65, 043408 (2002).

[83] R. S. Judson and H. Rabitz, "Teaching lasers to control molecules," Physical Review Letters 68, 1500-1503 (1992).

[84] J. Konradi, A. K. Singh, and A. Materny, "Selective excitation of molecular modes in a mixture by optimal control of electronically nonresonant femtosecond four-wave mixing spectroscopy," Journal of Photochemistry and Photobiology A: Chemistry 180, 289-299 (2006).

[85] S. D. McGrane, R. J. Scharff, M. Greenfield, and D. S. Moore, "Coherent control of multiple vibrational excitations for optimal detection," New Journal of Physics 11, 105047 (2009).

[86] A. M. Weiner, "Femtosecond pulse shaping using spatial light modulators," Review of Scientific Instruments 71, 1929-1960 (2000).

[87] A. Ostermeier, A. Gawelczyk, and N. Hansen, "Step-size adaptation based on non-local use of selection information," Lecture Notes in Computer Science 1141, 189-198 (1996).

[88] W. M. Sears, J. L. Hunt, and J. R. Stevens, "Raman spectra at low temperatures and depolarization ratios for styrene and polystyrene," Journal of Chemical Physics 77, 1639-1644 (1982).

[89] J. J. Max and C. Chapados, "Isotope effects in liquid water by infrared spectroscopy. III. H2O and D2O spectra from 6000 to $0 \mathrm{~cm}-1$," Journal of Chemical Physics 131, 184505 (2009).

[90] B. N. S. Inc., 12K Linear Array Spatial Light Modulator User Manual Rev 1.0 (Boulder Nonlinear Systems Inc., Lafayette CO, 2006). 
[91] C. R. I. Inc., SLM Spatial Light Modulator User's Manual Rev. 1.5 (Cambridge Research \& Instrumentation Inc., Woburn MA, 2006).

[92] J. W. Wilson, P. Schlup, M. Lunacek, D. Whitley, and R. A. Bartels, "Calibration of liquid crystal ultrafast pulse shaper with common-path spectral interferometry and application to coherent control with a covariance matrix adaptation evolutionary strategy," Review of Scientific Instruments 79, 033103 (2008).

[93] F. P. Ferreira and M. S. Belsley, "Direct calibration of a spatial light modulator by lateral shearing interferometry," Optics Express 18, 78997904 (2010).

[94] R. Trebino, Frequency-resolved optical gating: the measurement of ultrashort laser pulses (Kluwer Academic Publishers, Norwell MA, 2000).

[95] D. J. Kane and R. Trebino, "Characterization of arbitrary femtosecond pulses using frequency-resolved optical gating," IEEE Journal of Quantum Electronics 29, 571-579 (1993).

[96] R. Trebino, K. W. DeLong, D. N. Fittinghoff, J. N. Sweetser, M. A. Krumbügel, B. A. Richman, and D. J. Kane, "Measuring ultrashort laser pulses in the time-frequency domain using frequency-resolved optical gating," Review of Scientific Instruments 68, 3277-3295 (1997). 


\section{Summary}

This thesis describes improvements of chemical selectivity and contrast in coherent Raman scattering (CRS) spectroscopy and microscopy. Stimulated Raman scattering (SRS) and coherent anti-Stokes Raman scattering (CARS) provide chemical information of a sample based on the vibrational resonances in molecules. These resonances occur at characteristic frequencies related to the mass of the atoms and the strength of the bonds between the atoms. By detecting these resonances the presence of compounds of interest can be inferred. CRS microscopy is unaffected by one-photon fluorescence, offers label-free, non-invasive, chemically selective detection, and is inherently confocal.

In conventional narrowband CRS, the contrast is based on a narrow frequency range, covering only a single vibrational resonance. In samples with many compounds, vibrational resonances can overlap significantly and contrast based on a single resonance may not be sufficient to separate different compounds. Furthermore, CARS suffers from a non-resonant background that is generated even in the absence of vibrational resonances. This non-resonant background can overwhelm the resonant CARS signal. The CRS techniques described in this thesis excite multiple vibrational resonances simultaneously. Spectral phase shaping of the excitation light is used to influence the interferences between the different resonances and excitation pathways. In this way selective and background-free images are obtained.

Chapter two introduces the theory for two CRS processes, stimulated Raman scattering (SRS) and coherent anti-Stokes Raman scattering (CARS). Conventional narrowband implementations are covered as well 
as broadband techniques. The use of spectral phase shaping to obtain spectroscopic information in broadband CARS is demonstrated numerically.

Most narrowband SRS approaches rely on modulation of one of the input beams and lock-in detection on the other beam. Lock-in detection on spectrally resolved signals requires an array of lock-in detectors, which is not commercially available yet. This prevents a similar approach to broadband SRS. Chapter three describes a new broadband SRS technique based on a common-path interferometer, capable of obtaining vibrational information over a broad bandwidth, without the need for wavelength scanning or lock-in detection.

Chapter four contains numerical examples of spectral phase shaping for CARS, using complex phase profiles. An evolutionary algorithm is used to optimize the phase profile to enhance or suppress CARS signals from different compounds by influencing the interferences between the different pathways. The possibility for background-free imaging of a single compound is demonstrated, as well as chemically selective imaging of a single compound in a sample containing multiple compounds with overlapping resonances. Furthermore, studies on the robustness of the optimization approach in the case of mixing in the focal volume and phase noise are presented.

Chapter five demonstrates experimental optimization of phase profiles and background-free CARS imaging of a single compound, comparing the experimental results to the numerical results from chapter four. Phase optimizations for chemically selective imaging in a sample of multiple compounds are presented. Chemically selective imaging of a single compound is shown in samples containing up to five compounds with overlapping resonances. 


\section{Samenvatting}

In dit proefschrift worden nieuwe ontwikkelingen voor coherente Raman verstrooiing (CRS) spectroscopie en microscopie gepresenteerd, die de chemische selectiviteit en het contrast verhogen. CRS technieken leveren chemisch specifieke informatie over een preparaat aan de hand van de vibrationele resonanties in moleculen. Deze resonanties komen voor op specifieke frequenties die afhankelijk zijn van de massa's van de atomen en de sterkte van de bindingen tussen de atomen. De aanwezigheid van materialen kan afgeleid worden uit de aanwezigheid van kenmerkende resonantie frequenties.

Het chemisch contrast in smalbandige CRS technieken is gebaseerd op een smalle frequentie band met de breedte van een enkele vibrationele resonantie. Tijdens het CARS proces wordt ook een niet-resonant achtergrond signaal gegenereerd. Dit niet-resonante signaal wordt zelfs in afwezigheid van vibrationele resonanties gegenereerd en kan het resonante signaal overstemmen. In het geval van een preparaat van meerdere materialen kunnen de vibrationele resonanties van de verschillende materialen overlappen en kan contrast gebaseerd op een enkele resonantie niet meer toereikend zijn. De breedbandige technieken in dit proefschrift slaan meerdere resonanties tegelijkertijd aan, waarbij spectrale fase wordt gebruikt om de interferenties tussen de verschillende resonanties en excitatie paden te beïnvloeden.

De theorie van twee CRS processen, gestimuleerde Raman verstrooiing (SRS) en coherente anti-Stokes Raman verstrooiing (CARS), wordt beschreven in hoofdstuk twee. Zowel de conventionele smalbandige implementaties als de breedbandige implementaties worden behandeld. Even- 
eens wordt beschreven hoe spectrale fase gebruikt kan worden om spectrale informatie van een preparaat te verkrijgen.

De meeste smalbandige SRS technieken maken gebruik van modulatie van één van de excitatie bundels, en lock-in detectie op de andere bundel. Om deze methode toe te passen op een spectraal opgeloste bundel is een rij van lock-in detectoren nodig, maar een dergelijke lock-in detector array is nog niet verkrijgbaar. Hoofdstuk drie beschrijft een breedbandige SRS techniek die is gebaseerd op een interferometer, om breedbandige SRS metingen te doen zonder lock-in detectie.

In hoofdstuk vier wordt spectrale fase vervorming voor CARS met complexere fase profielen numeriek geïllustreerd. Met een evolutionair algoritme wordt de fase van de excitatie puls geoptimaliseerd, bijvoorbeeld om het CARS signaal van specifieke materialen te maximaliseren of te onderdrukken. De mogelijkheid om een enkel materiaal af te beelden zonder niet-resonante achtergrond of om een enkel materiaal selectief af te beelden in een complex preparaat wordt gedemonstreerd.

In hoofdstuk vijf wordt de experimentele optimalisatie van fase profielen behandeld. De achtergrond-vrije afbeelding van een enkel materiaal wordt gedemonstreerd en de experimentele resultaten worden vergeleken met de numerieke resultaten uit hoofdstuk vier. Verder wordt de fase optimalisatie in complexere preparaten getoond en wordt de selectieve afbeelding van een enkel materiaal gedemonstreerd in preparaten die tot vijf verschillende materialen met overlappende resonanties bevatten. 


\section{Dankwoord}

In het boekje dat voor jullie ligt worden de resultaten van het wetenschappelijk onderzoek waar ik mij de afgelopen vier jaar mee bezig heb gehouden gepresenteerd. Uiteraard was de totstandkoming van dit werk niet mogelijk geweest zonder de hulp en steun van anderen.

Allereerst wil ik mijn begeleider Herman bedanken. Jouw manier van begeleiden was erg aangenaam. Met name het altijd binnen kunnen lopen om een probleem te bespreken of nieuwe resultaten te laten zien was erg prettig.

Jennifer, ook jouw begeleiding en input is onmisbaar geweest tijdens dit project. Naarmate het project vorderde raakte je steeds meer betrokken en kwam ook het coherent control aspect verder naar voren. De verschillende perspectieven van jou en Herman vulden elkaar goed aan.

Zoals het geval is bij vrijwel elk onderzoek bij de Optical Sciences groep, was ook dit onderzoek niet mogelijk geweest zonder de excellente technische ondersteuning van Frans en Jeroen. Jullie technische kennis en kunde was ook voor dit onderzoek van onschatbare waarde. Karen, dank je wel voor het uit handen nemen van allerlei administratief werk.

Ali, thank you for introducing me to the subject of search landscapes and their optimization and for a very fruitful collaboration over the past few years.

Gaby, your internship only lasted for 3 months, but you worked very hard and we managed to get the broadband SRS working, as shown in 
chapter 3. It was a lot of fun to work with you and thanks to you the challenges and requirements for this technique are now better understood.

Erwin, bedankt voor je werk aan de fiber amplifier en ook voor de hulp tijdens de verhuizing naar het nieuwe gebouw. Dankzij jouw hulp ging het afbreken, inpakken, en opnieuw opbouwen van de opstelling een stuk sneller.

Martin, bedankt voor vele nuttige discussies en ook voor de gezelligheid in het kantoor, in het lab en tijdens conferenties. Verspreid door dit werk zul je ongetwijfeld resultaten herkennen die zijn voortgekomen uit onze discussies.

Frank, bedankt voor je interesse in deze CARS techniek en je werk in het lab. Je leert snel met de opstelling te werken en ik heb er vertrouwen in dat je de CARS techniek nog veel verder kunt ontwikkelen.

Dirk Jan, Dan, Jorick, Divya, and Andrew, thank you for proofreading various parts of this thesis. Your efforts have contributed significantly to the readability of this thesis.

De sfeer in de vakgroep was altijd erg goed, waardoor het erg aangenaam werken was. Dank daarvoor aan iedereen binnen de Optical Sciences groep. Met name dank aan Martin en Dirk Jan voor de gezelligheid tijdens vele koffiepauzes en, samen met Dan en Erik, voor de nodige potjes tafeltennis. Robert, Martin, Oya, Di, and Dan, thanks for the nice atmosphere in the office in T10. It was also really nice to work in the office in the Carre building thanks to Ali, Divya, Martin, Gaby, and many students who have worked there.

Uiteraard was er naast het onderzoek ook ruimte voor enige ontspanning. Stefan, Richard, Dieter en Lammert, dank jullie wel voor de vele potjes toepen en de gezelligheid tijdens de lunchpauzes de afgelopen jaren.

Dank ook aan alle Musilon leden voor de gezellige Musilon repetities op maandagavond, vaak gevolgd door naborrelen in het theatercafé en de VB. En ook dank aan de inmiddels oud-Musili voor een scala van activiteiten, variërend van spelletjes-avonden tot barbecues.

Vele avonden en weekenden waren ook lang niet zo gezellig geweest zon- 
der het gamen, dan wel online of offline, in het gezelschap van Stefan, Jorrit, Remco en Michiel. Remco en Stefan, bedankt dat jullie mij als paranimf bijstaan tijdens mijn verdediging.

In de laatste paragraaf wil ik ten slotte mijn naaste familieleden bedanken voor hun steun. Ook aan eenieder die heeft bijgedragen gedurende de afgelopen jaren maar hier niet individueel genoemd wordt, bedankt.

Alexander 


\section{List of publications}

\section{Journal articles}

- A.C.W. van Rhijn, S. Postma, J.P. Korterik, J.L. Herek, and H.L. Offerhaus, "Chemically selective imaging by spectral phase shaping for broadband CARS around $3000 \mathrm{~cm}^{-1} "$, Journal of the Optical Society of America B, 26, 559-563, (2009).

- A.C.W. van Rhijn, H.L. Offerhaus, P. van der Walle, J.L. Herek, and A. Jafarpour, "Exploring, tailoring, and traversing the solution landscape of a phase-shaped CARS process", Optics Express, 18(3), 2695-2709, (2010).

- A.C.W. van Rhijn, A. Jafarpour, M. Jurna, H.L. Offerhaus, and J.L. Herek, "Coherent control of vibrational transitions: Discriminating molecules in mixtures", Faraday Discussions, 153, 227-235, (2011).

- A.C.W. van Rhijn, M. Jurna, A. Jafarpour, J.L. Herek, and H.L. Offerhaus, "Phase-shaping strategies for coherent anti-Stokes Raman scattering", Journal of Raman Spectroscopy, 42(10), 1859-1863, (2011).

- D. Yang, D.P. Sprünken, A.C.W. van Rhijn, J. Savolainen, T.L. Chen, H.L. Offerhaus, J.L. Herek, and A. Jafarpour, "Investigation of adaptive laser pulse shaping by direct estimation of group delay profile", Optics Communications, 284(15), 3748-3758, (2011).

- H.L. Offerhaus, E.T. Garbacik, A.C.W. van Rhijn, A.L. Fussell, and J.L. Herek, "Phase aspects of (broadband) stimulated Raman scattering", Reviews in Analytical Chemistry, 31(1), 1-6, (2012).

\section{Journal articles (in preparation)}

- A.C.W. van Rhijn, A. Jafarpour, J.P. Korterik, H.L. Offerhaus, and J.L. Herek, "Phasial profiling for molecular discrimination", In preparation 


\section{Proceeding articles}

- A.C.W. van Rhijn, S. Postma, J.P. Korterik, J.L. Herek, and H.L. Offerhaus, "Spectral phase shaping for high resolution CARS spectroscopy around $3000 \mathrm{~cm}^{-1}$ ", Proceedings of SPIE, Volume 7183 Multiphoton Microscopy in the Biomedical Sciences IX, 71830X, (2009).

- A.C.W. van Rhijn, A. Jafarpour, J.L. Herek, and H.L. Offerhaus, "Computational optimization of phase shaped CARS", Proceedings of SPIE, Volume 8226 Multiphoton Microscopy in the Biomedical Sciences XII, 82262B, (2012).

\section{Other articles}

- A.C.W. van Rhijn and H.L. Offerhaus, "Phase shaping for CARS microscopy and spectroscopy", Application note for Boulder Nonlinear Systems, (2010).

\section{Conference contributions (oral)}

- A.C.W. van Rhijn, S. Postma, J.P. Korterik, J.L. Herek, and H.L. Offerhaus, "Spectral phase shaping for high resolution CARS spectroscopy around $3000 \mathrm{~cm}^{-1}$ ", SPIE Photonics West, San Jose CA, USA (2009).

- A.C.W. van Rhijn, A. Jafarpour, E. Bosma, J.A. Alvarez-Chavez, J.P. Korterik, J.L. Herek, and H.L. Offerhaus, "Phase shaping strategies for CARS", 9th ECONOS conference, Bremen, Germany (2010).

- A.C.W. van Rhijn, M. Jurna, A. Jafarpour, J.L. Herek, and H.L. Offerhaus, "Evolutionary optimization of phase shaped CARS", SPIE Photonics West, San Francisco CA, USA (2012).

- A.C.W. van Rhijn, A. Jafarpour, J.P. Korterik, J.L. Herek, and H.L. Offerhaus, "Experimental evolutionary optimization of phase shaped CARS", 11th ECONOS conference, Aberdeen, United Kingdom (2012).

- A.C.W. van Rhijn, M. Jurna, J.L. Herek, A. Jafarpour, and H.L. Offerhaus, "Optimal control CARS spectroscopy", 6th International Workshop on Optimal Control of Quantum Dynamics: Theory and Experiment, Tegernsee, Germany (2010).

- H.L. Offerhaus, M. Jurna, A.C.W. van Rhijn, A. Jafarpour, and J.L. Herek, "Coherent control of vibrational transitions", 9th ECONOS conference, Bremen, Germany (2010).

- A.C.W. van Rhijn, P. van der Walle, H.L. Offerhaus, J.L. Herek, and A. Jafarpour, "Exploring, tailoring, and traversing the solution landscape of two nonlinear optical processes", Laser Optics Berlin 2010, Berlin, Germany (2010). 
- D. Yang, A.C.W. van Rhijn, H.L. Offerhaus, J.L. Herek, and A. Jafarpour, "Systematic study of delay-based coherent control", 10th European Conference on Atoms Molecules and Photons, Salamanca, Spain (2010).

- D. Yang, D.P. Sprünken, A.C.W. van Rhijn, P. van der Walle, T.L. Chen, H.L. Offerhaus, J.L. Herek, A. Jafarpour, "Systematic study of delay-based adaptive coherent control", 17th International Conference on Ultrafast Phenomena, Snowmass Village CO, USA (2010).

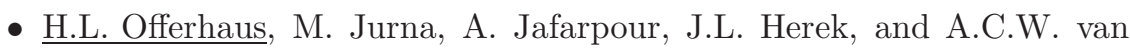
Rhijn, "Chemically selective imaging based on phase-shaped CARS", NWO meeting on Chemistry related to Physics \& Material Sciences/Dutch Polymer Days, Veldhoven, The Netherlands (2011).

- A.C.W. van Rhijn, E.T. Garbacik, A. Jafarpour, J.P. Korterik, H.L. Offerhaus, and J.L. Herek, "Optimal Control CARS Spectroscopy and Imaging", Gordon Research Conference on Quantum Control of Light and Matter, South Hadley MA, USA (2011).

\section{Conference contributions (poster)}

- A.C.W. van Rhijn, S. Postma, J.P. Korterik, J.L. Herek, and H.L. Offerhaus, "Shaped CARS", 32nd NNV AMO meeting, Lunteren, The Netherlands (2008).

- A.C.W. van Rhijn, S. Postma, M. Jurna, J.P. Korterik, J.A. AlvarezChavez, J.L. Herek, and H.L. Offerhaus, "Shaped CARS", Physics@FOM, Veldhoven, The Netherlands (2009).

- A.C.W. van Rhijn, A. Jafarpour, E. Bosma, J.A. Alvarez-Chavez, J.P. Korterik, J.L. Herek, and H.L. Offerhaus, "Phase shaping strategies for CARS", 33rd NNV AMO meeting, Lunteren, The Netherlands (2009).

- A.C.W. van Rhijn, A. Jafarpour, E. Bosma, J.A. Alvarez-Chavez, J.P. Korterik, J.L. Herek, and H.L. Offerhaus, "Phase shaping strategies for CARS", Physics@FOM, Veldhoven, The Netherlands (2010).

- A.C.W. van Rhijn, A. Jafarpour, E. Bosma, J.A. Alvarez-Chavez, J.P. Korterik, J.L. Herek, and H.L. Offerhaus, "Phase shaping strategies for CARS", 9th ECONOS conference, Bremen, Germany (2010).

- A.C.W. van Rhijn, M. Jurna, A. Jafarpour, H.L. Offerhaus, and J.L. Herek, "Phase shaping strategies for CARS", microCARS 2010, Bad Honnef, Germany (2010).

- A.C.W. van Rhijn, M. Jurna, A. Jafarpour, H.L. Offerhaus, and J.L. Herek, "Numerical study of phase-shaped CARS", NWO meeting on Chemistry related to Physics \& Material Sciences/Dutch Polymer Days, Veldhoven, The Netherlands (2011). 
- A.C.W. van Rhijn, M. Jurna, A. Jafarpour, H.L. Offerhaus, and J.L. Herek, "Numerical study of phase-shaped CARS", 10th ECONOS conference, Enschede, The Netherlands (2011).

- A.C.W. van Rhijn, M. Jurna, A. Jafarpour, H.L. Offerhaus, and J.L. Herek, "Numerical study of phase-shaped CARS", Gordon Research Conference on Quantum Control of Light and Matter, South Hadley MA, USA (2011).

- A.C.W. van Rhijn, M. Jurna, A. Jafarpour, H.L. Offerhaus, and J.L. Herek, "Numerical optimization of broadband CARS", 35th NNV AMO meeting, Lunteren, The Netherlands (2011).

- A.C.W. van Rhijn, M. Jurna, A. Jafarpour, H.L. Offerhaus, and J.L. Herek, "Numerical optimization of broadband CARS", Symposium on Photonic Integration and IOP-PD and STW-GTIP/SOS Program Meeting, Eindhoven, The Netherlands (2011).

- A.C.W. van Rhijn, A. Jafarpour, J.P. Korterik, H.L. Offerhaus, and J.L. Herek, "Phase optimization of broadband CARS", Physics@FOM, Veldhoven, The Netherlands (2012).

- A.C.W. van Rhijn, A. Jafarpour, J.P. Korterik, H.L. Offerhaus, and J.L. Herek, "Experimental evolutionary optimization of phase shaped CARS", 10th Nordic Femtochemistry Conference, Båstad, Sweden (2012).

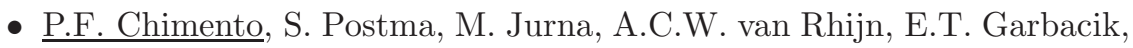
J.P. Korterik, C. Otto, J.L. Herek, and H.L. Offerhaus, "CARS spectroscopy and microscopy techniques", Annual MESA+ meeting, Enschede, The Netherlands (2008)

- D. Yang, J. Savolainen, A. Jafarpour, A.C.W. van Rhijn, H.L. Offerhaus, and J.L. Herek, "A new paradigm for photodynamic therapy: coherent control", 12th IPA "PDT: Back to the Future", Seattle WA, USA (2009).

- E.T. Garbacik, A.C.W. van Rhijn, M. Jurna, P.F. Chimento, J.P. Korterik, J.L Herek, and H.L. Offerhaus, "Background-free CARS techniques", NWO theory and spectroscopy, Lunteren, The Netherlands (2009)

- D. Yang, A.C.W. van Rhijn, T.L. Chen, D.J.W. Dikken, A. Jafarpour, H.L. Offerhaus, and J.L. Herek, "Pulse shaping spectroscopy", 10th ECONOS conference, Enschede, The Netherlands (2011).

\section{Colloquium contributions (oral)}

- A.C.W. van Rhijn, A. Jafarpour, E. Bosma, J.A. Alvarez-Chavez, J.P. Korterik, J.L. Herek, and H.L. Offerhaus, "Shaped CARS", Applied NanoPhotonics colloquium, Bad Bentheim, Germany (2010).

- A.C.W. van Rhijn, M. Jurna, A. Jafarpour, J.P. Korterik, H.L. Offerhaus, and J.L. Herek, "Shaped CARS", Physics colloquium at Lawrence University, Appleton WI, USA (2011). 


\section{Other presentations}

- H.L. Offerhaus and A.C.W. van Rhijn, "Holografie", Science Café Enschede, Enschede, The Netherlands, (2010). 

This thesis describes new techniques in coherent Raman scattering (CRS) spectroscopy and microscopy that improve chemical selectivity and specificity. Tailored broadband pulses are used to selectively excite molecules of interest and suppress unwanted contributions.

Alexander van Rhijn conducted his PhD research at the Optical Sciences group of the University of Twente in the Netherlands, where he developed broadband CRS techniques.



